Bull. Soc. math. France

134 (4), 2006, p. 559-631

\title{
CUTTING THE LOSS OF DERIVATIVES FOR SOLVABILITY UNDER CONDITION $(\Psi)$
}

\author{
BY NiCOLAS LERNER
}

\begin{abstract}
For a principal type pseudodifferential operator, we prove that condition $(\psi)$ implies local solvability with a loss of $3 / 2$ derivatives. We use many elements of Dencker's paper on the proof of the Nirenberg-Treves conjecture and we provide some improvements of the key energy estimates which allows us to cut the loss of derivatives from $\epsilon+3 / 2$ for any $\epsilon>0$ (Dencker's most recent result) to $3 / 2$ (the present paper). It is already known that condition $(\psi)$ does not imply local solvability with a loss of 1 derivative, so we have to content ourselves with a loss $>1$.

RÉSumé (Diminution de la perte de dérivées pour la résolubilité sous la condition $(\Psi)$ )

Pour un opérateur de type principal, nous démontrons que la condition $(\Psi)$ implique la résolubilité locale avec perte de $3 / 2$ dérivées. Nous utilisons beaucoup d'éléments de la démonstration par Dencker de la conjecture de Nirenberg-Treves et nous limitons la perte de dérivées à $3 / 2$, améliorant le résultat le plus récent de Dencker (perte de $\epsilon+3 / 2$ dérivées pour tout $\epsilon>0)$. La condition $(\Psi)$ n'impliquant pas la résolubilité locale avec perte d'une dérivée, nous devons nous contenter d'une perte $>1$.
\end{abstract}

\section{Introduction and statement of the results}

1.1. Introduction. - In 1957, Hans Lewy [25] constructed a counterexample showing that very simple and natural differential equations can fail to have

Texte reçu le 8 novembre 2005, accepté le 29 mars 2006.

Nicolas Lerner, Université Paris 6, Institut de Mathématiques de Jussieu, 175 rue du Chevaleret, 75013 Paris, France • E-mail : lerner@math.jussieu.fr

Url : http://www.math.jussieu.fr/ lerner/

2000 Mathematics Subject Classification. - 35S05, 47G30.

Key words and phrases. - Solvability, a priori estimates, pseudodifferential operators. 
local solutions; his example is the complex vector field $L_{0}=\partial_{x_{1}}+i \partial_{x_{2}}+i\left(x_{1}+\right.$ $\left.i x_{2}\right) \partial_{x_{3}}$ and one can show that there exists some $C^{\infty}$ function $f$ such that the equation $L_{0} u=f$ has no distribution solution, even locally. A geometric interpretation and a generalization of this counterexample were given in 1960 by L. Hörmander in [10] and extended in [11] to pseudodifferential operators. In 1970, L. Nirenberg and F. Treves ([29, 30, 31]), after a study of complex vector fields in [28] (see also [26]), refined this condition on the principal symbol to the so-called condition $(\psi)$, and provided strong arguments suggesting that it should be equivalent to local solvability. The necessity of condition $(\psi)$ for local solvability of pseudodifferential equations was proved in two dimensions by R. Moyer in [27] and in general by L. Hörmander ([13]) in 1981. The sufficiency of condition $(\psi)$ for local solvability of differential equations was proved by R. Beals and C. Fefferman ([1]) in 1973; they created a new type of pseudodifferential calculus, based on a Calderón-Zygmund decomposition, and were able to remove the analyticity assumption required by L. Nirenberg and F. Treves. For differential equations in any dimension ([1]) and for pseudodifferential equations in two dimensions ([18], see also [19]), it was shown more precisely that $(\psi)$ implies local solvability with a loss of one derivative with respect to the elliptic case: for a differential operator $P$ of order $m$ (or a pseudodifferential operator in two dimensions), satisfying condition $(\psi), f \in H_{\mathrm{loc}}^{s}$, the equation $P u=f$ has a solution $u \in H_{\mathrm{loc}}^{s+m-1}$. In 1994, it was proved by N.L. in [20] (see also [16], [24]) that condition $(\psi)$ does not imply local solvability with loss of one derivative for pseudodifferential equations, contradicting repeated claims by several authors. However in 1996, N. Dencker in [4], proved that these counterexamples were indeed locally solvable, but with a loss of two derivatives.

In [5], N. Dencker claimed that he can prove that condition $(\psi)$ implies local solvability with loss of two derivatives; this preprint contains several breakthrough ideas on the control of the second derivatives subsequent to condition $(\psi)$ and on the choice of the multiplier. The paper [7] contains a proof of local solvability with loss of two derivatives under condition $(\psi)$, providing the final step in the proof of the Nirenberg-Treves conjecture; the more recent paper [6] is providing a proof of local solvability with loss of $\epsilon+\frac{3}{2}$ derivatives under condition $(\psi)$, for any positive $\epsilon$. In the present article, we show that the loss can be limited to $3 / 2$ derivatives, dropping the $\epsilon$ in the previous result. We follow the pattern of Dencker's paper and give some improvements on the key energy estimates.

Acknowledgement. - For several months, I have had the privilege of exchanging several letters and files with Lars Hörmander on the topic of solvability. I am most grateful for the help generously provided. These personal communications are referred to in the text as [17] and are important in all sections of the present paper.

TOME $134-2006-\mathrm{N}^{\mathrm{O}} 4$ 
1.2. Statement of the result. - Let $P$ be a properly supported principaltype pseudodifferential operator in a $C^{\infty}$ manifold $\mathcal{M}$, with principal (complex-valued) ${ }^{(1)}$ symbol $p$. The symbol $p$ is assumed to be a $C^{\infty}$ homogeneous $^{(2)}$ function of degree $m$ on $\dot{T}^{*}(\mathcal{M})$, the cotangent bundle minus the zero section. The principal type assumption that we shall use here is that

$$
(x, \xi) \in \dot{T}^{*}(\mathcal{M}), \quad p(x, \xi)=0 \Longrightarrow \partial_{\xi} p(x, \xi) \neq 0 .
$$

Also, the operator $P$ will be assumed of polyhomogeneous type, which means that its total symbol is equivalent to $p+\sum_{j \geq 1} p_{m-j}$, where $p_{k}$ is a smooth homogeneous function of degree $k$ on $\dot{T}^{*}(\mathcal{M})$.

Definition 1.2.1 (Condition $(\psi)$ ). - Let $p$ be a $C^{\infty}$ homogeneous function on $\dot{T}^{*}(\mathcal{M})$. The function $p$ is said to satisfy condition $(\psi)$ if, for $z=1$ or $i$, Im $z p$ does not change sign from - to + along an oriented bicharacteristic of $\operatorname{Re} z p$.

It is a non-trivial fact that condition $(\psi)$ is invariant by multiplication by an complex-valued smooth elliptic factor (see section 26.4 in [14]).

THEOREM 1.2.2. — Let $P$ be as above, such that its principal symbol p satisfies condition $(\psi)$. Let $s$ be a real number. Then, for all $x \in \mathcal{M}$, there exists a neighborhood $V$ such that for all $f \in H_{l o c}^{s}$, there exists $u \in H_{l o c}^{s+m-\frac{3}{2}}$ such that

$$
P u=f \text { in } V \text {. }
$$

Proof. - The proof of this theorem will be given at the end of section 4 .

Note that our loss of derivatives is equal to $3 / 2$. The paper [20] proves that solvability with loss of one derivative does not follow from condition $(\psi)$, so we have to content ourselves with a loss strictly greater than one. However, the number $3 / 2$ is not likely to play any significant rôle and one should probably expect a loss of $1+\epsilon$ derivatives under condition $(\psi)$. In fact, for the counterexamples given in [20], it seems (but it has not been proven) that there is only a "logarithmic" loss, i.e., the solution $u$ should satisfy $u \in \log \left\langle D_{x}\right\rangle\left(H^{s+m-1}\right)$.

Nevertheless, the methods used in the present article are strictly limited to providing a $3 / 2$ loss. We refer the reader to our appendix A.4 for an argument involving a Hilbertian lemma on a simplified model. This is of course in sharp contrast with operators satisfying condition $(P)$ such as differential operators satisfying condition $(\psi)$. Let us recall that condition $(P)$ is simply ruling out any change of sign of $\operatorname{Im}(z p)$ along the oriented Hamiltonian flow of $\operatorname{Re}(z p)$. Under condition $(P)([1])$ or under condition $(\psi)$ in two dimensions $([18])$,

(1) Naturally the local solvability of real principal type operators is also a consequence of the next theorem, but much stronger results for real principal type equations were already established in the 1955 paper [9] (see also section 26.1 in [14]).

${ }^{(2)}$ Here and in the sequel, "homogeneous" will always mean positively homogeneous. 
local solvability occurs with a loss of one derivative, the "optimal" loss, and in fact the same as for $\partial / \partial x_{1}$. One should also note that the semi-global existence theorems of [12] (see also theorem 26.11.2 in [14]) involve a loss of $1+\epsilon$ derivatives. However in that case there is no known counterexample which would ensure that this loss is unavoidable.

REMARK 1.2.3. - Theorem 1.2.2 will be proved by a multiplier method, involving the computation of $\langle P u, M u\rangle$ with a suitably chosen operator $M$. It is interesting to notice that, the greater is the loss of derivatives, the more regular should be the multiplier in the energy method. As a matter of fact, the Nirenberg-Treves multiplier of [30] is not even a pseudodifferential operator in the $S_{1 / 2,1 / 2}^{0}$ class, since it could be as singular as the operator $\operatorname{sign} D_{x_{1}}$; this does not create any difficulty, since the loss of derivatives is only 1 . On the other hand, in [4], [23], where estimates with loss of 2 derivatives are handled, the regularity of the multiplier is much better than $S_{1 / 2,1 / 2}^{0}$, since we need to consider it as an operator of order 0 in an asymptotic class defined by an admissible metric on the phase space.

N.B. - For microdifferential operators acting on microfunctions, the sufficiency of condition $(\psi)$ was proven by J.-M. Trépreau [32] (see also [15]), so the present paper is concerned only with the $C^{\infty}$ category.

1.3. Some notations. - First of all, we recall the definition of the Weyl quantization $a^{w}$ of a function $a \in \mathcal{S}\left(\mathbb{R}^{2 n}\right)$ : for $u \in \mathcal{S}\left(\mathbb{R}^{n}\right)$,

$$
\left(a^{w} u\right)(x)=\iint e^{2 i \pi(x-y) \xi} a\left(\frac{x+y}{2}, \xi\right) u(y) d y .
$$

Our definition of the Fourier transform $\hat{u}$ of $u \in \mathcal{S}\left(\mathbb{R}^{n}\right)$ is $\hat{u}(\xi)=\int e^{-2 i \pi x \xi} u(x) d x$ and the usual quantization $a\left(x, D_{x}\right)$ of $a \in \mathcal{S}\left(\mathbb{R}^{2 n}\right)$ is $\left(a\left(x, D_{x}\right) u\right)(x)=$ $\int e^{2 i \pi x \xi} a(x, \xi) \hat{u}(\xi) d \xi$. The phase space $\mathbb{R}_{x}^{n} \times \mathbb{R}_{\xi}^{n}$ is a symplectic vector space with the standard symplectic form

$$
[(x, \xi),(y, \eta)]=\langle\xi, y\rangle-\langle\eta, x\rangle .
$$

Definition 1.3.1. - Let $g$ be a metric on $\mathbb{R}^{2 n}$, i.e., a mapping $X \mapsto g_{X}$ from $\mathbb{R}^{2 n}$ to the cone of positive definite quadratic forms on $\mathbb{R}^{2 n}$. Let $M$ be a positive function defined on $\mathbb{R}^{2 n}$.

(1) The metric $g$ is said to be slowly varying whenever $\exists C>0, \exists r>0$, $\forall X, Y, T \in \mathbb{R}^{2 n}$,

$$
g_{X}(Y-X) \leq r^{2} \Longrightarrow C^{-1} g_{Y}(T) \leq g_{X}(T) \leq C g_{Y}(T) .
$$

(2) The symplectic dual metric $g^{\sigma}$ is defined as $g_{X}^{\sigma}(T)=\sup _{g_{X}(U)=1}[T, U]^{2}$. The parameter of $g$ is defined as $\lambda_{g}(X)=\inf _{T \neq 0}\left(g_{X}^{\sigma}(T) / g_{X}(T)\right)^{1 / 2}$ and we shall say that $g$ satisfies the uncertainty principle if $\inf _{X} \lambda_{g}(X) \geq 1$.

TOME $134-2006-\mathrm{N}^{\mathrm{O}} 4$ 
(3) The metric $g$ is said to be temperate when $\exists C>0, \exists N \geq 0, \forall X, Y, T \in$ $\mathbb{R}^{2 n}$,

$$
g_{X}^{\sigma}(T) \leq C g_{Y}^{\sigma}(T)\left(1+g_{X}^{\sigma}(X-Y)\right)^{N}
$$

When the three properties above are satisfied, we shall say that $g$ is admissible. The constants appearing in (1) and (3) will be called the structure constants of the metric $g$.

(4) The function $M$ is said to be $g$-slowly varying if $\exists C>0, \exists r>0, \forall X, Y \in$ $\mathbb{R}^{2 n}$,

$$
g_{X}(Y-X) \leq r^{2} \Longrightarrow C^{-1} \leq \frac{M(X)}{M(Y)} \leq C .
$$

(5) The function $M$ is said to be $g$-temperate if $\exists C>0, \exists N \geq 0, \forall X, Y \in$ $\mathbb{R}^{2 n}$,

$$
\frac{M(X)}{M(Y)} \leq C\left(1+g_{X}^{\sigma}(X-Y)\right)^{N}
$$

When $M$ satisfies (4) and (5), we shall say that $M$ is a $g$-weight.

DeFinition 1.3.2. - Let $g$ be a metric on $\mathbb{R}^{2 n}$ and $M$ be a positive function defined on $\mathbb{R}^{2 n}$. The set $S(M, g)$ is defined as the set of functions $a \in C^{\infty}\left(\mathbb{R}^{2 n}\right)$ such that, for all $l \in \mathbb{N}$, $\sup _{X}\left\|a^{(l)}(X)\right\|_{g_{X}} M(X)^{-1}<\infty$, where $a^{(l)}$ is the $l$-th derivative. It means that $\forall l \in \mathbb{N}, \exists C_{l}, \forall X \in \mathbb{R}^{2 n}, \forall T_{1}, \ldots, T_{l} \in \mathbb{R}^{2 n}$,

$$
\left|a^{(l)}(X)\left(T_{1}, \ldots, T_{l}\right)\right| \leq C_{l} M(X) \prod_{1 \leq j \leq l} g_{X}\left(T_{j}\right)^{1 / 2} .
$$

REMARK. - If $g$ is a slowly varying metric and $M$ is $g$-slowly varying, there exists $M_{*} \in S(M, g)$ such that there exists $C>0$ depending only on the structure constants of $g$ such that

$$
\forall X \in \mathbb{R}^{2 n}, \quad C^{-1} \leq \frac{M_{*}(X)}{M(X)} \leq C .
$$

That remark is classical and its proof is sketched in the appendix A.2.

1.4. Partitions of unity. - We refer the reader to the chapter 18 in [14] for the basic properties of admissible metrics as well as for the following lemma.

LEMMA 1.4.1. - Let $g$ be an admissible metric on $\mathbb{R}^{2 n}$. There exists a sequence $\left(X_{k}\right)_{k \in \mathbb{N}}$ of points in the phase space $\mathbb{R}^{2 n}$ and positive numbers $r_{0}, N_{0}$, such that the following properties are satisfied. We define $U_{k}, U_{k}^{*}, U_{k}^{* *}$ as the $g_{k}=g_{X_{k}}$ balls with center $X_{k}$ and radius $r_{0}, 2 r_{0}, 4 r_{0}$. There exist two families of non-negative smooth functions on $\mathbb{R}^{2 n},\left(\chi_{k}\right)_{k \in \mathbb{N}},\left(\psi_{k}\right)_{k \in \mathbb{N}}$ such that

$$
\sum_{k} \chi_{k}(X)=1, \operatorname{supp} \chi_{k} \subset U_{k}, \quad \psi_{k} \equiv 1 \text { on } U_{k}^{*}, \operatorname{supp} \psi_{k} \subset U_{k}^{* *} \text {. }
$$

BULLETIN DE LA SOCiÉTÉ MATHÉMATIQUe DE FRANCE 
Moreover, $\chi_{k}, \psi_{k} \in S\left(1, g_{k}\right)$ with semi-norms bounded independently of $k$. The overlap of the balls $U_{k}^{* *}$ is bounded, i.e., $\bigcap_{k \in \mathcal{N}} U_{k}^{* *} \neq \varnothing \Rightarrow \# \mathcal{N} \leq N_{0}$. Moreover, $g_{X} \sim g_{k}$ all over $U_{k}^{* *}$ (i.e., the ratios $g_{X}(T) / g_{k}(T)$ are bounded above and below by a fixed constant, provided that $\left.X \in U_{k}^{* *}\right)$.

The next lemma in proved in [2] (see also lemma 6.3 in [22]).

LEMMA 1.4.2. - Let $g$ be an admissible metric on $\mathbb{R}^{2 n}$ and $\sum_{k} \chi_{k}(x, \xi)=1$ be a partition of unity related to $g$ as in the previous lemma. There exists a positive constant $C$ such that for all $u \in L^{2}\left(\mathbb{R}^{n}\right)$

$$
C^{-1}\|u\|_{L^{2}\left(\mathbb{R}^{n}\right)}^{2} \leq \sum_{k}\left\|\chi_{k}^{w} u\right\|_{L^{2}\left(\mathbb{R}^{n}\right)}^{2} \leq C\|u\|_{L^{2}\left(\mathbb{R}^{n}\right)}^{2}
$$

where $a^{w}$ stands for the Weyl quantization of the symbol a.

The following lemma is proved in [3].

LEMMA 1.4.3. - Let $g$ be an admissible metric on $\mathbb{R}^{2 n}, m$ be a weight for $g$, $U_{k}$ and $g_{k}$ as in lemma 1.4.1. Let $\left(a_{k}\right)$ be a sequence of bounded symbols in $S\left(m\left(X_{k}\right), g_{k}\right)$ such that, for all non-negative integers $l, N$

$$
\sup _{k \in \mathbb{N}, T \in \mathbb{R}^{2 n}}\left|m\left(X_{k}\right)^{-1} a_{k}^{(l)}(X) T^{l}\left(1+g_{k}^{\sigma}\left(X-U_{k}\right)\right)^{N} g_{k}(T)^{-l / 2}\right|<+\infty .
$$

Then the symbol $a=\sum_{k} a_{k}$ makes sense and belongs to $S(m, g)$. The important point here is that no support condition is required for the $a_{k}$, but instead some decay estimates with respect to $g^{\sigma}$. The sequence $\left(a_{k}\right)$ will be called a confined sequence in $S(m, g)$.

\section{The geometry of condition $(\psi)$}

In this section and also in section 3 , we shall consider that the phase space is equipped with a symplectic quadratic form $\Gamma$ ( $\Gamma$ is a positive definite quadratic form such that $\Gamma=\Gamma^{\sigma}$, see the definition 1.3.1(2) above). It is possible to find some linear symplectic coordinates $(x, \xi)$ in $\mathbb{R}^{2 n}$ such that

$$
\Gamma(x, \xi)=|(x, \xi)|^{2}=\sum_{1 \leq j \leq n} x_{j}^{2}+\xi_{j}^{2} .
$$

The running point of our Euclidean symplectic $\mathbb{R}^{2 n}$ will be usually denoted by $X$ or by an upper-case letter such as $Y, Z$. The open $\Gamma$-ball with center $X$ and radius $r$ will be denoted by $B(X, r)$.

TOME $134-2006-\mathrm{N}^{\mathrm{O}} 4$ 
2.1. The basic structure. - Let $q(t, X, \Lambda)$ be a smooth real-valued function defined on $\Xi=\mathbb{R} \times \mathbb{R}^{2 n} \times[1,+\infty)$, vanishing for $|t| \geq 1$ and satisfying

$$
\begin{gathered}
\forall k \in \mathbb{N}, \sup _{\Xi}\left\|\partial_{X}^{k} q\right\|_{\Gamma} \Lambda^{-1+\frac{k}{2}}=\gamma_{k}<+\infty, \text { i.e., } q(t, \cdot) \in S\left(\Lambda, \Lambda^{-1} \Gamma\right), \\
s>t \text { and } q(t, X, \Lambda)>0 \Longrightarrow q(s, X, \Lambda) \geq 0 .
\end{gathered}
$$

Notation. - In this section and in the next section, the Euclidean norm $\Gamma(X)^{1 / 2}$ is fixed and the norms of the vectors and of the multilinear forms are taken with respect to that norm. We shall write everywhere $|\cdot|$ instead of $\|\cdot\|_{\Gamma}$. Furthermore, we shall say that $C$ is a "fixed" constant if it depends only on a finite number of $\gamma_{k}$ above and on the dimension $n$.

We shall always omit the dependence of $q$ with respect to the large parameter $\Lambda$ and write $q(t, X)$ instead of $q(t, X, \Lambda)$. The operator $Q(t)=q(t)^{w}$ will stand for the operator with Weyl symbol $q(t, X)$. We introduce now for $t \in \mathbb{R}$, following [17],

$$
\begin{gathered}
\mathbb{X}_{+}(t)=\cup_{s \leq t}\left\{X \in \mathbb{R}^{2 n}, q(s, X)>0\right\}, \\
\mathbb{X}_{-}(t)=\cup_{s \geq t}\left\{X \in \mathbb{R}^{2 n}, q(s, X)<0\right\}, \\
\mathbb{X}_{0}(t)=\mathbb{X}_{-}(t)^{c} \cap \mathbb{X}_{+}(t)^{c},
\end{gathered}
$$

Thanks to (2.1.2), $\mathbb{X}_{+}(t), \mathbb{X}_{-}(t)$ are disjoint open subsets of $\mathbb{R}^{2 n}$; moreover $\mathbb{X}_{0}(t), \mathbb{X}_{0}(t) \cup \mathbb{X}_{ \pm}(t)$ are closed since their complements are open. The three sets $\mathbb{X}_{0}(t), \mathbb{X}_{ \pm}(t)$ are two by two disjoint with union $\mathbb{R}^{2 n}$ (note also that $\overline{\mathbb{X}_{ \pm}(t)} \subset$ $\mathbb{X}_{0}(t) \cup \mathbb{X}_{ \pm}(t)$ since $\mathbb{X}_{0}(t) \cup \mathbb{X}_{ \pm}(t)$ are closed). When $t$ increases, $\mathbb{X}_{+}(t)$ increases and $\mathbb{X}_{-}(t)$ decreases.

Lemma 2.1.1. - Let $(E, d)$ be a metric space, $A \subset E$ and $\kappa>0$ be given. We define $\Psi_{A, \kappa}(x)=\kappa$ if $A=\varnothing$ and if $A \neq \varnothing$, we define $\Psi_{A, \kappa}(x)=$ $\min (d(x, A), \kappa)$. The function $\Psi_{A, \kappa}$ is valued in $[0, \kappa]$, Lipschitz continuous with a Lipschitz constant $\leq 1$. Moreover, the following implication holds: $A_{1} \subset A_{2} \subset E \Longrightarrow \Psi_{A_{1}, \kappa} \geq \Psi_{A_{2}, \kappa}$.

Proof. - The Lipschitz continuity assertion is obvious since $x \mapsto d(x, A)$ is Lipschitz continuous with Lipschitz constant 1 . The monotonicity property is trivially inherited from the distance function.

Lemma 2.1.2. - For each $X \in \mathbb{R}^{2 n}$, the function $t \mapsto \Psi_{\mathbb{X}_{+}(t), \kappa}(X)$ is decreasing and for each $t \in \mathbb{R}$, the function $X \mapsto \Psi_{\mathbb{X}_{+}(t), \kappa}(X)$ is supported in $\mathbb{X}_{+}(t)^{c}=\mathbb{X}_{-}(t) \cup \mathbb{X}_{0}(t)$. For each $X \in \mathbb{R}^{2 n}$, the function $t \mapsto \Psi_{\mathbb{X}_{-}(t), \kappa}(X)$ is increasing and for each $t \in \mathbb{R}$, the function $X \mapsto \Psi_{\mathbb{X}_{-}(t), \kappa}(X)$ is supported in $\mathbb{X}_{-}(t)^{c}=\mathbb{X}_{+}(t) \cup \mathbb{X}_{0}(t)$. As a consequence the function $X \mapsto$ $\Psi_{\mathbb{X}_{+}(t), \kappa}(X) \Psi_{\mathbb{X}_{-}(t), \kappa}(X)$ is supported in $\mathbb{X}_{0}(t)$. 
Proof. — The monotonicity in $t$ follows from the fact that $\mathbb{X}_{+}(t)\left(\right.$ resp. $\left.\mathbb{X}_{-}(t)\right)$ is increasing (resp. decreasing) with respect to $t$ and from Lemma 2.1.1. Moreover, if $X$ belongs to the open set $\mathbb{X}_{ \pm}(t)$, one has $\Psi_{\mathbb{X}_{ \pm}(t), \kappa}(X)=0$, implying the support property.

Lemma 2.1.3. - For $\kappa>0, t \in \mathbb{R}, X \in \mathbb{R}^{2 n}$, we define w $^{(3)}$

$$
\sigma(t, X, \kappa)=\Psi_{\mathbb{X}_{-}(t), \kappa}(X)-\Psi_{\mathbb{X}_{+}(t), \kappa}(X) .
$$

The function $t \mapsto \sigma(t, X, \kappa)$ is increasing and valued in $[-\kappa, \kappa]$, the function $X \mapsto \sigma(t, X, \kappa)$ is Lipschitz continuous with Lipschitz constant less than 2 ; we have

$$
\sigma(t, X, \kappa)= \begin{cases}\min \left(\left|X-\mathbb{X}_{-}(t)\right|, \kappa\right) & \text { if } X \in \mathbb{X}_{+}(t), \\ -\min \left(\left|X-\mathbb{X}_{+}(t)\right|, \kappa\right) & \text { if } X \in \mathbb{X}_{-}(t) .\end{cases}
$$

We have $\left\{X \in \mathbb{R}^{2 n}, \sigma(t, X, \kappa)=0\right\} \subset \mathbb{X}_{0}(t) \subset\left\{X \in \mathbb{R}^{2 n}, q(t, X)=0\right\}$, and

$$
\begin{aligned}
\left\{X \in \mathbb{R}^{2 n},\right. & \pm q(t, X)>0\} \subset \mathbb{X}_{ \pm}(t) \subset\left\{X \in \mathbb{R}^{2 n}, \pm \sigma(t, X, \kappa)>0\right\} \\
& \subset\left\{X \in \mathbb{R}^{2 n}, \pm \sigma(t, X, \kappa) \geq 0\right\} \subset\left\{X \in \mathbb{R}^{2 n}, \pm q(t, X) \geq 0\right\} .
\end{aligned}
$$

Proof. - Everything follows from the previous lemmas, except for the first, fourth and sixth inclusions. Note that if $X \in \mathbb{X}_{+}(t), \sigma(t, X, \kappa)=$ $\min \left(\left|X-\mathbb{X}_{-}(t)\right|, \kappa\right)$ is positive (otherwise it vanishes and $X \in \mathbb{X}_{+}(t) \cap \overline{\mathbb{X}_{-}(t)} \subset$ $\left.\mathbb{X}_{+}(t) \cap\left(\mathbb{X}_{-}(t) \cup \mathbb{X}_{0}(t)\right)=\varnothing\right)$. As a consequence, we get the penultimate inclusions $\mathbb{X}_{+}(t) \subset\left\{X \in \mathbb{R}^{2 n}, \sigma(t, X, \kappa)>0\right\}$ and similarly $\mathbb{X}_{-}(t) \subset\left\{X \in \mathbb{R}^{2 n}, \sigma(t, X, \kappa)<0\right\}$, so that

$$
\left\{X \in \mathbb{R}^{2 n}, \sigma(t, X, \kappa)=0\right\} \subset \mathbb{X}_{+}(t)^{c} \cap \mathbb{X}_{-}(t)^{c}=\mathbb{X}_{0}(t),
$$

giving the first inclusion. The last inclusion follows from the already established

$$
\left.\left\{X \in \mathbb{R}^{2 n}, q(t, X)\right)<0\right\} \subset \mathbb{X}_{-}(t) \subset\left\{X \in \mathbb{R}^{2 n}, \sigma(t, X, \kappa)<0\right\} .
$$

Definition 2.1.4. — Let $q(t, X)$ be as above. We define

$$
\delta_{0}(t, X)=\sigma\left(t, X, \Lambda^{1 / 2}\right)
$$

and we notice that from the previous lemmas, $t \mapsto \delta_{0}(t, X)$ is increasing, valued in $\left[-\Lambda^{1 / 2}, \Lambda^{1 / 2}\right]$, satisfying

$$
\left|\delta_{0}(t, X)-\delta_{0}(t, Y)\right| \leq 2|X-Y|
$$

and such that

$$
\begin{gathered}
\left\{X \in \mathbb{R}^{2 n}, \delta_{0}(t, X)=0\right\} \subset\left\{X \in \mathbb{R}^{2 n}, q(t, X)=0\right\} \\
\left\{X \in \mathbb{R}^{2 n}, \pm q(t, X)>0\right\} \subset\left\{X, \pm \delta_{0}(t, X)>0\right\} \subset\{X, \pm q(t, X) \geq 0\}
\end{gathered}
$$

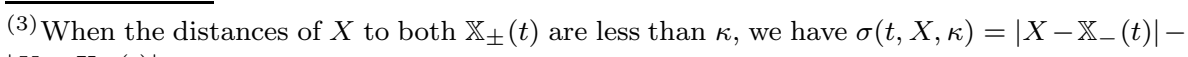
$\left|X-\mathbb{X}_{+}(t)\right|$.

TOME $134-2006-\mathrm{N}^{\mathrm{O}} 4$ 
LEMMA 2.1.5. - Let $f$ be a symbol in $S\left(\Lambda^{m}, \Lambda^{-1} \Gamma\right)$ where $m$ is a positive real number. We define

$$
\lambda(X)=1+\max _{\substack{0 \leq j<2 m \\ j \in \mathbb{N}}}\left(\left\|f^{(j)}(X)\right\|_{\Gamma}^{\frac{2}{2 m-j}}\right) .
$$

Then $f \in S\left(\lambda^{m}, \lambda^{-1} \Gamma\right)$ and the mapping from $S\left(\Lambda^{m}, \Lambda^{-1} \Gamma\right)$ to $S\left(\lambda^{m}, \lambda^{-1} \Gamma\right)$ is continuous. Moreover, with $\gamma=\max _{\substack{0 \leq j<2 m \\ j \in \mathbb{N}}} \gamma_{j}^{\frac{2}{2 m-j}}$, where the $\gamma_{j}$ are the seminorms of $f$, we have for all $X \in \mathbb{R}^{2 n}$,

$$
1 \leq \lambda(X) \leq 1+\gamma \Lambda
$$

The metric $\lambda^{-1} \Gamma$ is admissible(def. 1.3.1), with structure constants depending only on $\gamma$. It will be called the m-proper metric of $f$. The function $\lambda$ above is a weight for the metric $\lambda^{-1} \Gamma$ and will be called the $m$-proper weight of $f$.

Proof. - The proof of this lemma is given in the appendix A.3.

Lemma 2.1.6. - Let $q(t, X)$ and $\delta_{0}(t, X)$ be as above. We define, with $\langle s\rangle=$ $\left(1+s^{2}\right)^{1 / 2}$,

$$
\mu(t, X)=\left\langle\delta_{0}(t, X)\right\rangle^{2}+\left|\Lambda^{1 / 2} q_{X}^{\prime}(t, X)\right|+\left|\Lambda^{1 / 2} q_{X X}^{\prime \prime}(t, X)\right|^{2} .
$$

The metric $\mu^{-1}(t, \cdot) \Gamma$ is slowly varying with structure constants depending only on a finite number of semi-norms of $q$ in $S\left(\Lambda, \Lambda^{-1} \Gamma\right)$. Moreover, there exists $C>0$, depending only on a finite number of semi-norms of $q$, such that

$$
\mu(t, X) \leq C \Lambda, \quad \frac{\mu(t, X)}{\mu(t, Y)} \leq C\left(1+|X-Y|^{2}\right),
$$

and we have

$$
\Lambda^{1 / 2} q(t, X) \in S\left(\mu(t, X)^{3 / 2}, \mu^{-1}(t, \cdot) \Gamma\right),
$$

so that the semi-norms depend only the semi-norms of $q$ in $S\left(\Lambda, \Lambda^{-1} \Gamma\right)$.

Proof. — We notice first that

$$
1+\max \left(\left|\Lambda^{1 / 2} q_{X}^{\prime}(t, X)\right|,\left|\Lambda^{1 / 2} q_{X X}^{\prime \prime}(t, X)\right|^{2}\right)
$$

is the 1-proper weight of the vector-valued symbol $\Lambda^{1 / 2} q_{X}^{\prime}(t, \cdot)$. Using the lemma A.2.2, we get that $\mu^{-1} \Gamma$ is slowly varying, and the lemma A.2.1 provides the second part of (2.1.14). From the definition 2.1.4 and (2.1.1), we obtain that $\mu(t, X) \leq C \Lambda+\left\langle\delta_{0}(t, X)\right\rangle^{2} \leq C^{\prime} \Lambda$ and $\Lambda^{1 / 2} q_{X}^{\prime}(t, \cdot) \in S\left(\mu(t, X), \mu^{-1}(t, \cdot) \Gamma\right)$.

We are left with the proof of $\left|\Lambda^{1 / 2} q(t, X)\right| \leq C \mu^{3 / 2}(t, X)$. Let us consider $\widetilde{\mu}(t, X)$ the $3 / 2$-proper weight of $\Lambda^{1 / 2} q(t, X)$ :

$$
\tilde{\mu}(t, X)=1+\max _{j=0,1,2}\left|\Lambda^{1 / 2} q^{(j)}(t, X)\right|^{\frac{2}{3-j}},
$$

BULlETIN DE LA SOCIÉtÉ MATHÉMATIQUE DE FRANCE 
where all the derivatives are taken with respect to $X$; if the maximum is realized for $j \in\{1,2\}$, we get from Lemma 2.1.5 and (2.1.15) that

$$
\begin{aligned}
\left|\Lambda^{1 / 2} q(t, X)\right| \leq \widetilde{\mu}(t, X)^{3 / 2}=\left(1+\max _{j=1,2}\left|\Lambda^{1 / 2} q^{(j)}(t, X)\right|^{\frac{2}{3-j}}\right)^{\frac{3}{2}} & \\
& \leq\left(1+\max _{j=1,2} \mu^{\left(\frac{3}{2}-\frac{j}{2}\right)\left(\frac{2}{3-j}\right)}\right)^{\frac{3}{2}} \leq(2 \mu(t, X))^{3 / 2},
\end{aligned}
$$

which is the result that we had to prove. We have eventually to deal with the case where the maximum in the definition of $\widetilde{\mu}$ is realized for $j=0$; note that if $\widetilde{\mu}(t, X) \leq C_{0}$, we obtain

$$
\left|\Lambda^{1 / 2} q(t, X)\right| \leq \widetilde{\mu}(t, X)^{3 / 2} \leq C_{0}^{3 / 2} \leq C_{0}^{3 / 2} \mu(t, X)^{3 / 2},
$$

so we may also assume $\widetilde{\mu}(t, X)>C_{0}$. If $C_{0}>1$, we have $C_{0}<\widetilde{\mu}(t, X)=$ $1+\left(\Lambda^{1 / 2}|q(t, X)|\right)^{\frac{2}{3}}$ entailing

$$
\left(1-C_{0}^{-1}\right) \widetilde{\mu}(t, X) \leq\left|\Lambda^{1 / 2} q(t, X)\right|^{\frac{2}{3}} \leq \widetilde{\mu}(t, X) .
$$

Now if $h \in \mathbb{R}^{2 n}$ is such that $|h| \leq r \widetilde{\mu}(t, X)^{1 / 2}$, we get from the slow variation of the metric $\widetilde{\mu}^{-1} \Gamma$, that the ratio $\widetilde{\mu}(t, X+h) / \widetilde{\mu}(t, X)$ is bounded above and below, provided $r$ is small enough. Using now that $\Lambda^{1 / 2} q(t, \cdot) \in$ $S\left(\widetilde{\mu}^{3 / 2}(t, \cdot), \widetilde{\mu}^{-1}(t, \cdot) \Gamma\right)$, we get by Taylor's formula

$\Lambda^{1 / 2} q(t, X+h)=\Lambda^{1 / 2} q(t, X)+\Lambda^{1 / 2} q^{\prime}(t, X) h+\frac{1}{2} \Lambda^{1 / 2} q^{\prime \prime}(t, X) h^{2}+O\left(\gamma_{3}|h|^{3} / 6\right)$,

so that

$$
\begin{aligned}
\Lambda^{1 / 2}|q(t, X+h)| & \geq \Lambda^{1 / 2}|q(t, X)|-\widetilde{\mu}(t, X)|h|-\frac{1}{2}|h|^{2} \widetilde{\mu}(t, X)^{1 / 2}-\gamma_{3}|h|^{3} / 6 \\
& \geq \Lambda^{1 / 2}|q(t, X)|-\widetilde{\mu}(t, X)^{3 / 2} \underbrace{\left(r+\frac{r^{2}}{2}+\gamma_{3} \frac{r^{3}}{6}\right)}_{=\epsilon(r)} .
\end{aligned}
$$

This gives $\Lambda^{1 / 2}|q(t, X+h)| \geq \Lambda^{1 / 2}|q(t, X)|-\epsilon(r) \widetilde{\mu}(t, X)^{3 / 2}, \lim _{r \rightarrow 0} \epsilon(r)=0$, so that, for $r, C_{0}^{-1}$ small enough,

$$
\left|\Lambda^{1 / 2} q(t, X+h)\right| \geq\left(\left(1-C_{0}^{-1}\right)^{3 / 2}-\epsilon(r)\right) \widetilde{\mu}(t, X)^{3 / 2} \geq \frac{1}{2} \widetilde{\mu}(t, X)^{3 / 2} .
$$

As a consequence, the $\Gamma$-ball $B\left(X, r \widetilde{\mu}(t, X)^{1 / 2}\right)$ is included in $\mathbb{X}_{+}(t)$ or in $\mathbb{X}_{-}(t)$ and thus, in the first case (the second case is similar) $\left|X-\mathbb{X}_{+}(t)\right|=$ $0,\left|X-\mathbb{X}_{-}(t)\right| \geq r \widetilde{\mu}(t, X)^{1 / 2}$, (otherwise $\left|X-\mathbb{X}_{-}(t)\right|<r \widetilde{\mu}(t, X)^{1 / 2}$ and $\left.\varnothing \neq B\left(X, r \widetilde{\mu}(t, X)^{1 / 2}\right) \cap \mathbb{X}_{-}(t) \subset \mathbb{X}_{+}(t) \cap \mathbb{X}_{-}(t)=\varnothing\right)$, implying that, with a fixed $r_{0}>0$,

$$
\delta_{0}(t, X) \geq \min \left(\Lambda^{1 / 2}, r \widetilde{\mu}(t, X)^{1 / 2}\right) \geq r_{0} \widetilde{\mu}(t, X)^{1 / 2} \geq r_{0}\left|\Lambda^{1 / 2} q(t, X)\right|^{1 / 3},
$$

so that, in both cases, $\left|\Lambda^{1 / 2} q(t, X)\right| \leq r_{0}^{-3}\left|\delta_{0}(t, X)\right|^{3} \leq r_{0}^{-3} \mu(t, X)^{3 / 2}$.

TOME $134-2006-\mathrm{N}^{\mathrm{O}} 4$ 
Lemma 2.1.7. - Let $q(t, X), \delta_{0}(t, X), \mu(t, X)$ be as above. We define,

$$
\nu(t, X)=\left\langle\delta_{0}(t, X)\right\rangle^{2}+\left|\Lambda^{1 / 2} q_{X}^{\prime}(t, X) \mu(t, X)^{-1 / 2}\right|^{2} .
$$

The metric $\nu^{-1}(t, \cdot) \Gamma$ is slowly varying with structure constants depending only on a finite number of semi-norms of $q$ in $S\left(\Lambda, \Lambda^{-1} \Gamma\right)$. There exists $C>0$, depending only on a finite number of semi-norms of $q$, such that

$$
\nu(t, X) \leq 2 \mu(t, X) \leq C \Lambda, \quad \frac{\nu(t, X)}{\nu(t, Y)} \leq C\left(1+|X-Y|^{2}\right),
$$

and we have

$$
\Lambda^{1 / 2} q(t, X) \in S\left(\mu(t, X)^{1 / 2} \nu(t, X), \nu(t, \cdot)^{-1} \Gamma\right),
$$

so that the semi-norms of this symbol depend only the semi-norms of $q$ in $S\left(\Lambda, \Lambda^{-1} \Gamma\right)$. Moreover the function $\mu(t, X)$ is a weight for the metric $\nu(t, \cdot)^{-1} \Gamma$.

Proof. - Let us check the two first inequalities in (2.1.17). From $\left|\Lambda^{1 / 2} q^{\prime}\right| \leq$ $\mu(t, X) \leq C \Lambda$, established in the previous lemma, we get

$$
\nu(t, X) \leq\left\langle\delta_{0}(t, X)\right\rangle^{2}+\mu(t, X) \leq 2 \mu(t, X) \leq 2 C \Lambda .
$$

We introduce now the weight $\mu_{*}(t, X)$ as in (1.3.3) so that the ratios $\mu_{*}(t, X) / \mu(t, X)$ are bounded above and below by some constants depending only on a finite number of semi-norms of $q$. That weight $\mu_{*}(t, X)$ belongs to $S\left(\mu, \mu^{-1} \Gamma\right)=S\left(\mu_{*}, \mu_{*}^{-1} \Gamma\right)$. We notice first that

$$
\begin{aligned}
\left|\Lambda^{1 / 2}\left(q \mu_{*}^{-1 / 2}\right)^{\prime}\right|^{2} \leq 2 \mid \Lambda^{1 / 2} q^{\prime} & \left.\mu_{*}^{-1 / 2}\right|^{2}+C_{1}\left|\Lambda^{1 / 2} q \mu^{-1}\right|^{2} \\
& \leq C_{2}\left|\Lambda^{1 / 2} q^{\prime} \mu^{-1 / 2}\right|^{2}+C_{1}\left|\Lambda^{1 / 2} q \mu^{-1 / 2}\right| \overbrace{\left|\Lambda^{1 / 2} q \mu^{-3 / 2}\right|}^{\lesssim 1} \\
& \leq C_{2}\left|\Lambda^{1 / 2} q^{\prime} \mu^{-1 / 2}\right|^{2}+C_{3}\left|\Lambda^{1 / 2} q \mu^{-1 / 2}\right| .
\end{aligned}
$$

Since we have also ${ }^{(4)}$

$$
\begin{aligned}
\left|\Lambda^{1 / 2} q^{\prime} \mu^{-1 / 2}\right| \sim\left|\Lambda^{1 / 2} q^{\prime} \mu_{*}^{-1 / 2}\right| & \lesssim\left|\Lambda^{1 / 2}\left(q \mu_{*}^{-1 / 2}\right)^{\prime}\right|+\left|\Lambda^{1 / 2} q \mu_{*}^{-1}\right| \\
& \lesssim\left|\Lambda^{1 / 2}\left(q \mu_{*}^{-1 / 2}\right)^{\prime}\right|+\underbrace{\left|\Lambda^{1 / 2} q \mu_{*}^{-3 / 2}\right|^{1 / 2}}_{\lesssim 1}\left|\Lambda^{1 / 2} q \mu_{*}^{-1 / 2}\right|^{1 / 2}
\end{aligned}
$$

we get that

$$
\widetilde{\nu}(t, X)=1+\max \left(\left|\Lambda^{1 / 2} q_{X}^{\prime}(t, X) \mu(t, X)^{-1 / 2}\right|^{2},\left|\Lambda^{1 / 2} q(t, X) \mu(t, X)^{-1 / 2}\right|\right)
$$

is equivalent to the 1 -proper weight of the symbol $\Lambda^{1 / 2} q(t, X) \mu_{*}(t, X)^{-1 / 2}$ in $S\left(\mu, \mu^{-1} \Gamma\right)$. As a consequence, from the lemma A.2.2, we get that $\left(\widetilde{\nu}+\left\langle\delta_{0}\right\rangle^{2}\right)^{-1} \Gamma$ is slowly varying.

(4) Below, the inequality $a \lesssim b$ means that $a \leq C b$ where $C$ is a constant depending only on a finite number of semi-norms of $q$. The equivalence $a \sim b$ stands for $a \lesssim b$ and $b \lesssim a$.

BULLETIN DE LA SOCIÉTÉ MATHÉMATIQUe DE FRANCE 
$\triangleright$ We need only to prove that

$$
\left.\mid \Lambda^{1 / 2} q(t, X)\right) \mu(t, X)^{-1 / 2} \mid \leq C \nu(t, X) .
$$

In fact, from (2.1.20), we shall obtain $\nu(t, X) \leq \widetilde{\nu}(t, X)+\left\langle\delta_{0}(t, X)\right\rangle^{2} \leq$ $(C+1) \nu(t, X)$ so that the metrics $\left(\widetilde{\nu}+\left\langle\delta_{0}\right\rangle^{2}\right)^{-1} \Gamma$ and $\nu^{-1} \Gamma$ are equivalent and thus both slowly varying (that property will also give the last inequality in (2.1.17) from Lemma A.2.1). Moreover, from Lemma 2.1.5, we have $\Lambda^{1 / 2} q(t, X) \mu_{*}(t, X)^{-1 / 2} \in S\left(\widetilde{\nu}, \widetilde{\nu}^{-1} \Gamma\right)$, so that

$$
\Lambda^{1 / 2}\left(q \mu_{*}^{-1 / 2}\right)^{(k)} \lesssim \begin{cases}\nu^{1-k / 2} & \text { for } k \leq 2, \\ & \text { since } \Lambda^{1 / 2} q \mu_{*}^{-1 / 2} \in S\left(\widetilde{\nu} ;, \widetilde{\nu}^{-1} \Gamma\right) \text { and } \widetilde{\nu} \lesssim \nu, \\ \mu^{1-k / 2} \lesssim \nu^{1-k / 2} & \text { for } k \geq 2, \\ & \text { since } \Lambda^{1 / 2} q \mu_{*}^{-1 / 2} \in S\left(\mu ; \mu^{-1} \Gamma\right) \text { and } \nu \lesssim \mu\end{cases}
$$

which implies that $\Lambda^{1 / 2} q \mu_{*}^{-1 / 2} \in S\left(\nu, \nu^{-1} \Gamma\right)$; moreover, we have $\mu_{*}^{1 / 2} \in$ $S\left(\mu_{*}^{1 / 2}, \nu^{-1} \Gamma\right)$ since, using $\nu \lesssim \mu$, we get

$$
\left|\left(\mu_{*}^{1 / 2}\right)^{(k)}\right| \lesssim \mu^{\frac{1-k}{2}} \lesssim \mu^{\frac{1}{2}} \nu^{-k / 2}
$$

entailing $\Lambda^{1 / 2} q \in S\left(\mu^{1 / 2} \nu, \nu^{-1} \Gamma\right)$, i.e., (2.1.18). On the other hand, $\mu$ is slowly varying for $\nu^{-1} \Gamma$, since

$$
|X-Y| \ll \nu(t, X)^{1 / 2}\left(\lesssim \mu(t, X)^{1 / 2}\right) \quad \text { implies }|X-Y| \ll \mu(t, X)^{1 / 2}
$$

and thus $\mu(t, X) \sim \mu(t, Y)$, which proves along with (2.1.14) that $\mu$ is a weight for $\nu^{-1} \Gamma$.

$\triangleright$ Let us now check (2.1.20). This inequality is obvious if $\left|\Lambda^{1 / 2} q \mu^{-1 / 2}\right| \leq$ $\left|\Lambda^{1 / 2} q^{\prime} \mu^{-1 / 2}\right|^{2}$. Note that if $\widetilde{\nu}(t, X) \leq C_{0}$, we obtain $\left|\Lambda^{1 / 2} q \mu^{-1 / 2}\right| \leq C_{0} \leq C_{0} \nu$ so we may also assume $\widetilde{\nu}(t, X)>C_{0}$. If $C_{0}>1$, we have $C_{0}<\widetilde{\nu}(t, X)=$ $1+\left(\Lambda^{1 / 2}|q| \mu^{-1 / 2}\right)$ entailing

$$
\left(1-C_{0}^{-1}\right) \widetilde{\nu}(t, X) \leq\left|\Lambda^{1 / 2} q \mu^{-1 / 2}\right| \leq \widetilde{\nu}(t, X) .
$$

Now if $h \in \mathbb{R}^{2 n}$ is such that $|h| \leq r \widetilde{\nu}(t, X)^{1 / 2}$, we get from the slow variation of the metric $\widetilde{\nu}^{-1} \Gamma$, that the ratio $\widetilde{\nu}(t, X+h) / \widetilde{\nu}(t, X)$ is bounded above and below, provided $r$ is small enough. Using now that $\Lambda^{1 / 2} q \mu_{*}^{-1 / 2} \in S\left(\widetilde{\nu}, \widetilde{\nu}^{-1} \Gamma\right)$, we get by Taylor's formula

$$
\Lambda^{1 / 2} q(t, X+h) \mu_{*}^{-1 / 2}(t, X+h)=\Lambda^{1 / 2} q(t, X) \mu_{*}^{-1 / 2}(t, X)+\epsilon(r) \widetilde{\nu}(t, X),
$$

$\lim _{r \rightarrow 0} \epsilon(r)=0$, so that, for $r, C_{0}^{-1}$ small enough,

$$
\left|\Lambda^{1 / 2} q(t, X+h) \mu_{*}^{-1 / 2}(t, X+h)\right| \geq\left(\left(1-C_{0}^{-1}\right)-\epsilon(r)\right) \widetilde{\nu}(t, X) \geq \frac{1}{2} \widetilde{\nu}(t, X) .
$$

As a consequence, the $\Gamma$-ball $B\left(X, r \widetilde{\nu}(t, X)^{1 / 2}\right)$ is included in $\mathbb{X}_{+}(t)$ or in $\mathbb{X}_{-}(t)$ and thus, in the first case (the second case is similar) $\left|X-\mathbb{X}_{+}(t)\right|=0$, 
$\left|X-\mathbb{X}_{-}(t)\right| \geq r \widetilde{\nu}(t, X)^{1 / 2}$, implying that, with a fixed $r_{0}>0$,

$$
\begin{aligned}
\delta_{0}(t, X) \geq \min \left(\Lambda^{1 / 2}, r \widetilde{\nu}(t, X)^{1 / 2}\right) & \geq r_{0} \widetilde{\nu}(t, X)^{1 / 2} \\
& \geq r_{0}\left|\Lambda^{1 / 2} q(t, X) \mu(t, X)^{-1 / 2}\right|^{1 / 2},
\end{aligned}
$$

so that, in both cases, $\left|\Lambda^{1 / 2} q(t, X) \mu(t, X)^{-1 / 2}\right| \leq C_{0}\left|\delta_{0}(t, X)\right|^{2} \leq C_{0} \nu(t, X)$. The proof of the lemma is complete.

We wish now to discuss the normal forms attached to the metric $\nu^{-1}(t, \cdot) \Gamma$ for the symbol $q(t, \cdot)$. In the sequel of this section, we consider that $t$ is fixed.

Definition 2.1.8. - Let $0<r_{1} \leq 1 / 2$ be given. With $\nu$ defined in (2.1.16), we shall say that

(1) $Y$ is a nonnegative (resp. nonpositive) point at level $t$ if $\delta_{0}(t, Y) \geq$ $r_{1} \nu(t, Y)^{1 / 2},\left(\right.$ resp. $\left.\delta_{0}(t, Y) \leq-r_{1} \nu(t, Y)^{1 / 2}\right)$.

(2) $Y$ is a gradient point at level $t$ if $\left|\Lambda^{1 / 2} q_{Y}^{\prime}(t, Y) \mu(t, Y)^{-1 / 2}\right|^{2} \geq$ $\nu(t, Y) / 4$ and $\delta_{0}(t, Y)^{2}<r_{1}^{2} \nu(t, Y)$.

(3) $Y$ is a negligible point in the remaining cases $\left|\Lambda^{1 / 2} q_{Y}^{\prime}(t, Y) \mu(t, Y)^{-1 / 2}\right|^{2}<$ $\nu(t, Y) / 4$ and $\delta_{0}(t, Y)^{2}<r_{1}^{2} \nu(t, Y)$. Note that this implies $\nu(t, Y) \leq$ $1+r_{1}^{2} \nu(t, Y)+\nu(t, Y) / 4 \leq 1+\nu(t, Y) / 2$ and thus $\nu(t, Y) \leq 2$.

Note that if $Y$ is a nonnegative point, from (2.1.8) we get, for $T \in \mathbb{R}^{2 n}$, $|T| \leq 1,0 \leq r \leq r_{1} / 4$

$$
\delta_{0}\left(t, Y+r \nu^{1 / 2}(t, Y) T\right) \geq \delta_{0}(t, Y)-2 r \nu^{1 / 2}(t, Y) \geq \frac{r_{1}}{2} \nu^{1 / 2}(t, Y)
$$

and from (2.1.10), this implies that $q(t, X) \geq 0$ on the ball $B\left(Y, r \nu^{1 / 2}(t, Y)\right)$. Similarly if $Y$ is a nonpositive point, $q(t, X) \leq 0$ on the ball $B\left(Y, r \nu^{1 / 2}(t, Y)\right)$. Moreover if $Y$ is a gradient point, we may assume that $B\left(Y, r_{1}^{3} \nu^{1 / 2}(t, Y)\right)$ intersects $\{X, q(t, X)=0\}$, otherwise it is included either in $\{X, q(t, X)>0\}$ or in $\{X, q(t, X)<0\}$; as a result, there exists a point $Z \in B\left(Y, r_{1}^{3} \nu^{1 / 2}(t, Y)\right)$ such that $q(t, Z)=0$. The function

$$
f(T)=\Lambda^{1 / 2} q\left(t, Y+r_{1} \nu^{1 / 2}(t, Y) T\right) \mu(t, Y)^{-1 / 2} \nu(t, Y)^{-1}
$$

satisfies for $r_{1}$ small enough with respect to the semi-norms of $q$ and $c_{0}, C_{0}, C_{1}, C_{2}$ fixed positive constants, $|T| \leq 1$, from (2.1.18),

$$
\begin{gathered}
|f(0)|=\left|\Lambda^{1 / 2} q(t, Y) \mu(t, Y)^{-1 / 2} \nu(t, Y)^{-1}\right| \\
\leq \Lambda^{1 / 2} \mu^{-1 / 2} \nu^{-1} \max _{X \in[Y, Z]}\left|q^{\prime}(t, X)\right||Y-Z| \leq C_{0} r_{1}^{3}, \\
\left|f^{\prime}(T)\right| \geq r_{1} c_{0}, \quad\left|f^{\prime \prime}(T)\right| \leq C_{2} r_{1}^{2} .
\end{gathered}
$$

BULLETIN DE LA SOCIÉtÉ MATHÉMATIQUE DE FRANCE 
The standard analysis (see our appendix A.6) of the Beals-Fefferman metric shows that, on $B\left(Y, r_{1} \nu^{1 / 2}(t, Y)\right)$

$$
\begin{gathered}
q(t, X)=\Lambda^{-1 / 2} \mu^{1 / 2}(t, Y) \nu^{1 / 2}(t, Y) e(t, X) \beta(t, X) \\
1 \leq e \in S\left(1, \nu(t, Y)^{-1} \Gamma\right), \beta \in S\left(\nu(t, Y)^{1 / 2}, \nu(t, Y)^{-1} \Gamma\right), \\
\beta(t, X)=\nu(t, Y)^{1 / 2}\left(X_{1}+\alpha\left(t, X^{\prime}\right)\right), \alpha \in S\left(\nu(t, Y)^{1 / 2}, \nu(t, Y)^{-1} \Gamma\right) .
\end{gathered}
$$

LEMMA 2.1.9. - Let $q(t, X)$ be a smooth function satisfying (2.1.1-2) and let $t \in[-1,1]$ be given. The metric $g_{t}$ on $\mathbb{R}^{2 n}$ is defined as $\nu(t, X)^{-1} \Gamma$ where $\nu$ is defined in (2.1.16). There exists $r_{0}>0$, depending only on a finite number of semi-norms of $q$ in (2.1.1) such that, for any $\left.r \in] 0, r_{0}\right]$, there exist a sequence of points $\left(X_{k}\right)$ in $\mathbb{R}^{2 n}$, and sequences of functions $\left(\chi_{k}\right),\left(\psi_{k}\right)$ satisfying the properties in the lemma 1.4 .1 such that there exists a partition of $\mathbb{N}$,

$$
\mathbb{N}=E_{+} \cup E_{-} \cup E_{0} \cup E_{00}
$$

so that, according to the definition 2.1.8, $k \in E_{+}$means that $X_{k}$ is a nonnegative point, $\left(k \in E_{-}: X_{k}\right.$ nonpositive point; $k \in E_{0}: X_{k}$ gradient point, $k \in E_{00}: X_{k}$ negligible point).

Proof. - This lemma is an immediate consequence of the definition 2.1.8, of lemma 1.4.1 and of lemma 2.1.7, asserting that the metric $g_{t}$ is admissible.

2.2. Some lemmas on $\boldsymbol{C}^{\mathbf{3}}$ functions. - We prove in this section a key result on the second derivative $f_{X X}^{\prime \prime}$ of a real-valued smooth function $f(t, X)$ such that $\tau-i f(t, x, \xi)$ satisfies condition $(\psi)$. The following claim gives a good qualitative version of what is needed for our estimates; we shall not use this result, so the reader may skip the proof and proceed directly to the more technical Lemma 2.2.2.

Claim 2.2.1. - Let $f_{1}, f_{2}$ be two real-valued twice differentiable functions defined on an open set $\Omega$ of $\mathbb{R}^{N}$ and such that $f_{1}^{-1}\left(\mathbb{R}_{+}^{*}\right) \subset f_{2}^{-1}\left(\mathbb{R}_{+}\right)$(i.e., $f_{1}(x)>$ $\left.0 \Longrightarrow f_{2}(x) \geq 0\right)$. If for some $\omega \in \Omega$, the conditions $f_{1}(\omega)=f_{2}(\omega)=$ $0, \quad d f_{1}(\omega) \neq 0, d f_{2}(\omega)=0$ are satisfied, we have $f_{2}^{\prime \prime}(\omega) \geq 0$ (as a quadratic form).

Proof. - Using the obvious invariance by change of coordinates of the statement, we may assume $f_{1}(x) \equiv x_{1}$ and $\omega=0$. The assumption is then for $x=\left(x_{1}, x^{\prime}\right) \in \mathbb{R} \times \mathbb{R}^{N-1}$ in a neighborhood of the origin

$$
f_{2}(0)=0, d f_{2}(0)=0, \quad x_{1}>0 \Longrightarrow f_{2}\left(x_{1}, x^{\prime}\right) \geq 0 .
$$

Using the second-order Taylor-Young formula for $f_{2}$, we get $f_{2}(x)=$ $\frac{1}{2}\left\langle f_{2}^{\prime \prime}(0) x, x\right\rangle+\epsilon(x)|x|^{2}, \lim _{x \rightarrow 0} \epsilon(x)=0$, and thus for $T=\left(T_{1}, T^{\prime}\right),|T|=1$, $\rho \neq 0$ small enough, the implication $T_{1}>0 \Longrightarrow\left\langle f_{2}^{\prime \prime}(0) T, T\right\rangle+2 \epsilon(\rho T) \geq 0$. Consequently we have $\left\{S,\left\langle f_{2}^{\prime \prime}(0) S, S\right\rangle \geq 0\right\} \supset\left\{S, S_{1}>0\right\}$ and since the larger

TOME $134-2006-\mathrm{N}^{\mathrm{O}} 4$ 
set is closed and stable by the symmetry with respect to the origin, we get that it contains also $\left\{S, S_{1} \leq 0\right\}$, which is the result $f_{2}^{\prime \prime}(0) \geq 0$.

REMARK. - This claim has the following consequence: take three functions $f_{1}, f_{2}, f_{3}$, twice differentiable on $\Omega$, such that, for $1 \leq j \leq k \leq 3, f_{j}(x)>$ $0 \Rightarrow f_{k}(x) \geq 0$. Assume that, at some point $\omega$ we have $f_{1}(\omega)=f_{2}(\omega)=$ $f_{3}(\omega)=0, \quad d f_{1}(\omega) \neq 0, d f_{3}(\omega) \neq 0, d f_{2}(\omega)=0$. Then one has $f_{2}^{\prime \prime}(\omega)=0$. The claim 2.2.1 gives $f_{2}^{\prime \prime}(\omega) \geq 0$ and it can be applied to the couple $\left(-f_{3},-f_{2}\right)$ to get $-f_{2}^{\prime \prime}(\omega) \geq 0$.

Notation. - The open Euclidean ball of $\mathbb{R}^{N}$ with center 0 and radius $r$ will be denoted by $B_{r}$. For a k-multilinear symmetric form $A$ on $\mathbb{R}^{N}$, we shall note $\|A\|=\max _{|T|=1}\left|A T^{k}\right|$ which is easily seen to be equivalent to the norm $\max _{\left|T_{1}\right|=\cdots=\left|T_{k}\right|=1}\left|A\left(T_{1}, \ldots, T_{k}\right)\right|$ since the symmetrized $T_{1} \otimes \cdots \otimes T_{k}$ can be written a sum of $k^{\text {th }}$ powers.

LEMMA 2.2.2. - Let $R_{0}>0$ and $f_{1}, f_{2}$ be real-valued functions defined in $\bar{B}_{R_{0}}$. We assume that $f_{1}$ is $C^{2}, f_{2}$ is $C^{3}$ and for $x \in \bar{B}_{R_{0}}$,

$$
f_{1}(x)>0 \Longrightarrow f_{2}(x) \geq 0 \text {. }
$$

We define the non-negative numbers $\rho_{1}, \rho_{2}$, by

$$
\rho_{1}=\max \left(\left|f_{1}(0)\right|^{\frac{1}{2}},\left|f_{1}^{\prime}(0)\right|\right), \quad \rho_{2}=\max \left(\left|f_{2}(0)\right|^{\frac{1}{3}},\left|f_{2}^{\prime}(0)\right|^{\frac{1}{2}},\left|f_{2}^{\prime \prime}(0)\right|\right),
$$

and we assume that, with a positive $C_{0}$,

$$
0<\rho_{1}, \quad \rho_{2} \leq C_{0} \rho_{1} \leq R_{0} .
$$

We define the non-negative numbers $C_{1}, C_{2}, C_{3}$, by

$$
C_{1}=1+C_{0}\left\|f_{1}^{\prime \prime}\right\|_{L^{\infty}\left(\bar{B}_{R_{0}}\right)}, \quad C_{2}=4+\frac{1}{3}\left\|f_{2}^{\prime \prime \prime}\right\|_{L^{\infty}\left(\bar{B}_{R_{0}}\right)}, \quad C_{3}=C_{2}+4 \pi C_{1} .
$$

Assume that for some $\kappa_{2} \in[0,1]$, with $\kappa_{2} C_{1} \leq 1 / 4$,

$$
\begin{gathered}
\rho_{1}=\left|f_{1}^{\prime}(0)\right|>0, \\
\max \left(\left|f_{2}(0)\right|^{1 / 3},\left|f_{2}^{\prime}(0)\right|^{1 / 2}\right) \leq \kappa_{2}\left|f_{2}^{\prime \prime}(0)\right|, \\
B\left(0, \kappa_{2}^{2} \rho_{2}\right) \cap\left\{x \in \bar{B}_{R_{0}}, f_{1}(x) \geq 0\right\} \neq \varnothing .
\end{gathered}
$$

Then we have

$$
\left|f_{2}^{\prime \prime}(0)_{-}\right| \leq C_{3} \kappa_{2} \rho_{2},
$$

where $f_{2}^{\prime \prime}(0)_{-}$stands for the negative part of the quadratic form $f_{2}^{\prime \prime}(0)$. Note that, whenever (2.2.7) is violated, we get $B\left(0, \kappa_{2}^{2} \rho_{2}\right) \subset\left\{x \in \bar{B}_{R_{0}}, f_{1}(x)<0\right\}$ (note that $\kappa_{2}^{2} \rho_{2} \leq \rho_{2} \leq R_{0}$ ) and thus

$$
\operatorname{distance}\left(0,\left\{x \in \bar{B}_{R_{0}}, f_{1}(x) \geq 0\right\}\right) \geq \kappa_{2}^{2} \rho_{2} \text {. }
$$

BULlETIN DE LA SOCiÉtÉ MATHÉMATIQUE DE FRANCE 
Proof. - We may assume that for $x=\left(x_{1}, x^{\prime}\right) \in \mathbb{R} \times \mathbb{R}^{N-1}, \rho_{1}=\left|f_{1}^{\prime}(0)\right|=$ $\frac{\partial f_{1}}{\partial x_{1}}(0,0), \frac{\partial f_{1}}{\partial x^{\prime}}(0,0)=0$, so that

$$
f_{1}(x) \geq f_{1}(0)+\rho_{1} x_{1}-\frac{1}{2}\left\|f_{1}^{\prime \prime}\right\|_{\infty}|x|^{2} .
$$

Moreover, from (2.2.7), we know that there exists $z \in B\left(0, \kappa_{2}^{2} \rho_{2}\right)$ such that $f_{1}(z) \geq 0$. As a consequence, we have $0 \leq f_{1}(z) \leq f_{1}(0)+\rho_{1} z_{1}+\frac{1}{2}\left\|f_{1}^{\prime \prime}\right\|_{\infty} \kappa_{2}^{4} \rho_{2}^{2}$ and thus

$$
f_{1}(x) \geq \rho_{1} x_{1}-\rho_{1} \kappa_{2}^{2} \rho_{2}-\frac{1}{2}\left\|f_{1}^{\prime \prime}\right\|_{\infty}\left(|x|^{2}+\kappa_{2}^{4} \rho_{2}^{2}\right) .
$$

On the other hand, we have

$$
\begin{aligned}
f_{2}(x) \leq f_{2}(0)+f_{2}^{\prime}(0) x+\frac{1}{2} f_{2}^{\prime \prime}(0) & x^{2}+\frac{1}{6}\left\|f_{2}^{\prime \prime \prime}\right\|_{\infty}|x|^{3} \\
& \leq \kappa_{2}^{3} \rho_{2}^{3}+\kappa_{2}^{2} \rho_{2}^{2}|x|+\frac{1}{6}\left\|f_{2}^{\prime \prime \prime}\right\|_{\infty}|x|^{3}+\frac{1}{2} f_{2}^{\prime \prime}(0) x^{2}
\end{aligned}
$$

and the implications, for $|x| \leq R_{0}$,

$$
\begin{aligned}
\rho_{1} x_{1}>\rho_{1} \kappa_{2}^{2} \rho_{2}+\frac{1}{2}\left\|f_{1}^{\prime \prime}\right\|_{\infty}\left(|x|^{2}+\kappa_{2}^{4} \rho_{2}^{2}\right) \Longrightarrow f_{1}(x)>0 \Longrightarrow \\
f_{2}(x) \geq 0 \Longrightarrow-\frac{1}{2} f_{2}^{\prime \prime}(0) x^{2} \leq \kappa_{2}^{3} \rho_{2}^{3}+\kappa_{2}^{2} \rho_{2}^{2}|x|+\frac{1}{6}\left\|f_{2}^{\prime \prime \prime}\right\|_{\infty}|x|^{3} .
\end{aligned}
$$

Let us take $x=\kappa_{2} \rho_{2} y$ with $|y|=1$ (note that $|x|=\kappa_{2} \rho_{2} \leq R_{0}$ ); the property (2.2.12) gives, using $\rho_{2} / \rho_{1} \leq C_{0}$,

$$
y_{1}>\kappa_{2}\left(1+\left\|f_{1}^{\prime \prime}\right\|_{\infty} C_{0}\right) \Longrightarrow-f_{2}^{\prime \prime}(0) y^{2} \leq \kappa_{2} \rho_{2}\left(4+\frac{1}{3}\left\|f_{2}^{\prime \prime \prime}\right\|_{\infty}\right),
$$

so that $\left\{y \in \mathbb{S}^{N-1},-f_{2}^{\prime \prime}(0) y^{2} \leq \kappa_{2} \rho_{2}\left(4+\frac{1}{3}\left\|f_{2}^{\prime \prime \prime}\right\|_{\infty}\right)\right\} \supset\left\{y \in \mathbb{S}^{N-1}, y_{1}>\right.$ $\left.\kappa_{2}\left(1+\left\|f_{1}^{\prime \prime}\right\|_{\infty} C_{0}\right)\right\}$ and since the larger set is closed and stable by symmetry with respect to the origin, we get, with

$$
C_{1}=1+\left\|f_{1}^{\prime \prime}\right\|_{\infty} C_{0}, C_{2}=4+\frac{1}{3}\left\|f_{2}^{\prime \prime \prime}\right\|_{\infty},
$$

the implication

$$
y \in \mathbb{S}^{N-1},\left|y_{1}\right| \geq \kappa_{2} C_{1} \Longrightarrow-f_{2}^{\prime \prime}(0) y^{2} \leq \kappa_{2} \rho_{2} C_{2} .
$$

Let us now take $y \in \mathbb{S}^{N-1}$, such that $\left|y_{1}\right|<\kappa_{2} C_{1}(\leq 1 / 4)$. We may assume $y=y_{1} \overrightarrow{e_{1}} \oplus y_{2} \overrightarrow{e_{2}}$, with $\overrightarrow{e_{1}}, \overrightarrow{e_{2}}$, orthogonal unit vectors and $y_{2}=\left(1-y_{1}^{2}\right)^{1 / 2}$. We consider the following rotation in the $\left(\overrightarrow{e_{1}}, \overrightarrow{e_{2}}\right)$ plane with $\epsilon_{0}=\kappa_{2} C_{1} \leq 1 / 4$, $R=\left(\begin{array}{cc}\cos \left(2 \pi \epsilon_{0}\right) & \sin \left(2 \pi \epsilon_{0}\right) \\ -\sin \left(2 \pi \epsilon_{0}\right) & \cos \left(2 \pi \epsilon_{0}\right)\end{array}\right)$, so that $\left|(R y)_{1}\right|=\left|y_{1} \cos \left(2 \pi \epsilon_{0}\right)+y_{2} \sin \left(2 \pi \epsilon_{0}\right)\right|$, and since $\epsilon_{0} \leq 1 / 4$,

$$
\left|(R y)_{1}\right| \geq-\left|y_{1}\right|+\left(1-y_{1}^{2}\right)^{1 / 2} 4 \epsilon_{0} \geq \epsilon_{0}(\sqrt{15}-1)>\epsilon_{0}=\kappa_{2} C_{1} .
$$

TOME $134-2006-\mathrm{N}^{\mathrm{O}} 4$ 
Moreover the rotation $R$ satisfies $\|R-\mathrm{Id}\| \leq 2 \pi \epsilon_{0}=2 \pi \kappa_{2} C_{1}$. We have, using (2.2.13) and $\left|(R y)_{1}\right| \geq \kappa_{2} C_{1},|y|=1$,

$$
\begin{aligned}
&-f_{2}^{\prime \prime}(0) y^{2}=-f_{2}^{\prime \prime}(0)(R y)^{2}-\left\langle f_{2}^{\prime \prime}(0)(y-R y), y+R y\right\rangle \\
& \leq-f_{2}^{\prime \prime}(0)(R y)^{2}+\left|f_{2}^{\prime \prime}(0)\right||y-R y||y+R y| \\
& \leq \kappa_{2} \rho_{2} C_{2}+2 \rho_{2}|y-R y| \leq \kappa_{2} \rho_{2} C_{2}+2 \rho_{2} 2 \pi \kappa_{2} C_{1} .
\end{aligned}
$$

Eventually, for all $y \in \mathbb{S}^{N-1}$, we have

$$
-f_{2}^{\prime \prime}(0) y^{2} \leq \kappa_{2} \rho_{2}\left(C_{2}+4 \pi C_{1}\right)=C_{3} \kappa_{2} \rho_{2} .
$$

Considering now the quadratic form $Q=f_{2}^{\prime \prime}(0)$ and its canonical decomposition $Q=Q_{+}-Q_{-}$, we have, for all $y \in \mathbb{R}^{N},\left\langle Q_{-} y, y\right\rangle \leq \kappa_{2} \rho_{2} C_{3}|y|^{2}+\left\langle Q_{+} y, y\right\rangle$. Using now the canonical orthogonal projections $E_{ \pm}$on the positive (resp. negative) eigenspaces, we write $y=E_{+} y \oplus E_{-} y$ and we get that

$$
\begin{aligned}
\left\langle Q_{-} y, y\right\rangle= & \left\langle Q_{-} E_{-} y, E_{-} y\right\rangle \\
& \leq C_{3} \kappa_{2} \rho_{2}\left|E_{-} y\right|^{2}+\left\langle Q_{+} E_{-} y, E_{-} y\right\rangle=C_{3} \kappa_{2} \rho_{2}\left|E_{-} y\right|^{2} \leq C_{3} \kappa_{2} \rho_{2}|y|^{2},
\end{aligned}
$$

yielding (2.2.8). The proof of Lemma 2.2.2 is complete.

LEMMA 2.2.3. - Let $f_{1}, f_{2}, f_{3}$ be real-valued functions defined in $\bar{B}_{R_{0}}$. We assume that $f_{1}, f_{3}$ are $C^{2}, f_{2}$ is $C^{3}$ and for $x \in \bar{B}_{R_{0}}, 1 \leq j \leq k \leq 3$,

$$
f_{j}(x)>0 \Longrightarrow f_{k}(x) \geq 0 \text {. }
$$

We define the non-negative numbers $\rho_{1}, \rho_{2}, \rho_{3}$ by

$$
\begin{aligned}
& \rho_{1}=\max \left(\left|f_{1}(0)\right|^{\frac{1}{2}},\left|f_{1}^{\prime}(0)\right|\right) \quad \rho_{2}=\max \left(\left|f_{2}(0)\right|^{\frac{1}{3}},\left|f_{2}^{\prime}(0)\right|^{\frac{1}{2}},\left|f_{2}^{\prime \prime}(0)\right|\right), \\
& \rho_{3}=\max \left(\left|f_{3}(0)\right|^{\frac{1}{2}},\left|f_{3}^{\prime}(0)\right|\right)
\end{aligned}
$$

and we assume that, with a positive $C_{0}$,

$$
0<\rho_{1}, \rho_{3} \text { and } \rho_{2} \leq C_{0} \min \left(\rho_{1}, \rho_{3}\right) \leq C_{0} \max \left(\rho_{1}, \rho_{3}\right) \leq R_{0} .
$$

We define the non-negative numbers $C_{1}, C_{2}, C_{3}$, by

$$
\begin{aligned}
& C_{1}=1+C_{0} \max \left(\left\|f_{1}^{\prime \prime}\right\|_{L^{\infty}\left(\bar{B}_{R_{0}}\right)},\left\|f_{3}^{\prime \prime}\right\|_{L^{\infty}\left(\bar{B}_{R_{0}}\right)}\right), \\
& C_{2}=4+\frac{1}{3}\left\|f_{2}^{\prime \prime \prime}\right\|_{L^{\infty}\left(\bar{B}_{R_{0}}\right)}, \quad C_{3}=C_{2}+4 \pi C_{1} .
\end{aligned}
$$

Assume that for some $\kappa_{1}, \kappa_{3} \in[0,1]$, and $0<\kappa_{2} C_{3} \leq 1 / 2$,

$$
\begin{gathered}
\left|f_{1}(0)\right|^{1 / 2} \leq \kappa_{1}\left|f_{1}^{\prime}(0)\right|, \quad\left|f_{3}(0)\right|^{1 / 2} \leq \kappa_{3}\left|f_{3}^{\prime}(0)\right|, \\
B\left(0, \kappa_{2}^{2} \rho_{2}\right) \cap\left\{x \in \bar{B}_{R_{0}}, f_{1}(x) \geq 0\right\} \neq \varnothing \\
B\left(0, \kappa_{2}^{2} \rho_{2}\right) \cap\left\{x \in \bar{B}_{R_{0}}, f_{3}(x) \leq 0\right\} \neq \varnothing .
\end{gathered}
$$

Then we have

$$
\max \left(\left|f_{2}(0)\right|^{1 / 3},\left|f_{2}^{\prime}(0)\right|^{1 / 2}\right) \leq \rho_{2} \leq \kappa_{2}^{-1} \max \left(\left|f_{2}(0)\right|^{1 / 3},\left|f_{2}^{\prime}(0)\right|^{1 / 2}\right) .
$$

BULLETIN DE LA SOCIÉtÉ MATHÉMATIQUE DE FRANCE 
Note that, whenever (2.2.20) or (2.2.21) is violated, we get

$$
B\left(0, \kappa_{2}^{2} \rho_{2}\right) \subset\left\{x \in \bar{B}_{R_{0}}, f_{1}(x)<0\right\} \text { or } B\left(0, \kappa_{2}^{2} \rho_{2}\right) \subset\left\{x \in \bar{B}_{R_{0}}, f_{3}(x)>0\right\}
$$

and thus

$(2.2 .23)$

$\operatorname{dist}\left(0,\left\{x \in \bar{B}_{R_{0}}, f_{1}(x) \geq 0\right\}\right) \geq \kappa_{2}^{2} \rho_{2}$ or $\operatorname{dist}\left(0,\left\{x \in \bar{B}_{R_{0}}, f_{3}(x) \leq 0\right\}\right) \geq \kappa_{2}^{2} \rho_{2}$.

Proof. - This follows almost immediately from the previous lemma and it is analogous to the remark following the claim 2.2.1: assuming that we have

$$
\max \left(\left|f_{2}(0)\right|^{1 / 3},\left|f_{2}^{\prime}(0)\right|^{1 / 2}\right) \leq \kappa_{2}\left|f_{2}^{\prime \prime}(0)\right|
$$

will yield $\left|f_{2}^{\prime \prime}(0)\right| \leq C_{3} \kappa_{2} \rho_{2}$ by applying lemma 2.2 .2 (note that $\kappa_{2} C_{1} \leq \kappa_{2} \frac{C_{3}}{4 \pi} \leq$ $\left.\frac{1}{8 \pi}<1 / 4\right)$ to the couples $\left(f_{1}, f_{2}\right)$ and $\left(-f_{3},-f_{2}\right)$; consequently, if $(2.2 .24)$ is satisfied, we get

$$
\max \left(\left|f_{2}(0)\right|^{1 / 3},\left|f_{2}^{\prime}(0)\right|^{1 / 2}\right) \leq \rho_{2} \leq \max \left(\left|f_{2}(0)\right|^{1 / 3},\left|f_{2}^{\prime}(0)\right|^{1 / 2}, C_{3} \kappa_{2} \rho_{2}\right)
$$

and since $C_{3} \kappa_{2}<1$, it yields

$$
\max \left(\left|f_{2}(0)\right|^{1 / 3},\left|f_{2}^{\prime}(0)\right|^{1 / 2}\right)=\rho_{2},
$$

which implies (2.2.22). Let us now suppose that (2.2.24) does not hold, and that we have $\kappa_{2}\left|f_{2}^{\prime \prime}(0)\right|<\max \left(\left|f_{2}(0)\right|^{1 / 3},\left|f_{2}^{\prime}(0)\right|^{1 / 2}\right)$. This implies (2.2.22):

$$
\max \left(\left|f_{2}(0)\right|^{1 / 3},\left|f_{2}^{\prime}(0)\right|^{1 / 2}\right) \leq \rho_{2} \leq \kappa_{2}^{-1} \max \left(\left|f_{2}(0)\right|^{1 / 3},\left|f_{2}^{\prime}(0)\right|^{1 / 2}\right) .
$$

The proof of the lemma is complete.

REMARK. - We shall apply this lemma to a "fixed" $\kappa_{2}$, depending only on the constant $C_{3}$ such as $\kappa_{2}=1 /\left(2 C_{3}\right)$.

2.3. Inequalities for symbols. - In this section, we apply the results of the previous section to obtain various inequalities on symbols linked to our symbol $q$ introduced in (2.1.1). Our main result is the following theorem.

THEOREM 2.3.1. - Let $q$ be a symbol satisfying (2.1.1-2) and $\delta_{0}, \mu, \nu$ as defined above in (2.1.7), (2.1.13) and (2.1.16). For the real numbers $t^{\prime}, t, t^{\prime \prime}$, and $X \in \mathbb{R}^{2 n}$, we define

$$
\begin{aligned}
N\left(t^{\prime}, t^{\prime \prime}, X\right) & =\frac{\left\langle\delta_{0}\left(t^{\prime}, X\right)\right\rangle}{\nu\left(t^{\prime}, X\right)^{1 / 2}}+\frac{\left\langle\delta_{0}\left(t^{\prime \prime}, X\right)\right\rangle}{\nu\left(t^{\prime \prime}, X\right)^{1 / 2}} \\
R(t, X) & =\Lambda^{-1 / 2} \mu(t, X)^{1 / 2} \nu(t, X)^{-1 / 2}\left\langle\delta_{0}(t, X)\right\rangle .
\end{aligned}
$$

Then there exists a constant $C_{0} \geq 1$, depending only on a finite number of semi-norms of $q$ in (2.1.1), such that, for $t^{\prime} \leq t \leq t^{\prime \prime}$, we have

$$
C_{0}^{-1} R(t, X) \leq N\left(t^{\prime}, t^{\prime \prime}, X\right)+\frac{\delta_{0}\left(t^{\prime \prime}, X\right)-\delta_{0}(t, X)}{\nu\left(t^{\prime \prime}, X\right)^{1 / 2}}+\frac{\delta_{0}(t, X)-\delta_{0}\left(t^{\prime}, X\right)}{\nu\left(t^{\prime}, X\right)^{1 / 2}} .
$$

TOME $134-2006-\mathrm{N}^{\mathrm{O}} 4$ 
Proof. - We are given $X \in \mathbb{R}^{2 n}$ and $t^{\prime} \leq t \leq t^{\prime \prime}$ real numbers.

$\triangleright$ First reductions. First of all, we may assume that, for some positive (small) $\kappa$ to be chosen later, we have

$$
\left\langle\delta_{0}\left(t^{\prime}, X\right)\right\rangle \leq \kappa \nu\left(t^{\prime}, X\right)^{1 / 2} \quad \text { and } \quad\left\langle\delta_{0}\left(t^{\prime \prime}, X\right)\right\rangle \leq \kappa \nu\left(t^{\prime \prime}, X\right)^{1 / 2} .
$$

In fact, otherwise, we have $N\left(t^{\prime}, t^{\prime \prime}, X\right)>\kappa$ and since from (2.1.14), we have $\mu(t, X) \leq C \Lambda$ where $C$ depends only on a finite number of semi-norms of $q$, we get from (2.3.2), (2.1.16)

$$
R(t, X) \leq C^{1 / 2} \nu(t, X)^{-1 / 2}\left\langle\delta_{0}(t, X)\right\rangle \leq C^{1 / 2} \leq C^{1 / 2} \kappa^{-1} N\left(t^{\prime}, t^{\prime \prime}, X\right),
$$

so that we shall only need

$$
C_{0} \geq C^{1 / 2} \kappa^{-1}
$$

to obtain (2.3.3). Also, we may assume that, with the same positive (small) $\kappa$,

$$
\nu(t, X) \leq \kappa^{2} \nu\left(t^{\prime}, X\right) \text { and } \nu(t, X) \leq \kappa^{2} \nu\left(t^{\prime \prime}, X\right) .
$$

Otherwise, we would have for instance $\nu(t, X)>\kappa^{2} \nu\left(t^{\prime}, X\right)$ and since $t \geq t^{\prime}$,

$$
\begin{aligned}
& R(t, X) \leq \Lambda^{-1 / 2} \mu(t, X)^{1 / 2} \kappa^{-1} \frac{\left\langle\delta_{0}(t, X)\right\rangle}{\nu\left(t^{\prime}, X\right)^{1 / 2}} \\
& \leq C^{1 / 2} \kappa^{-1}\left(\frac{\left\langle\delta_{0}\left(t^{\prime}, X\right)\right\rangle+|\overbrace{\delta_{0}(t, X)-\delta_{0}\left(t^{\prime}, X\right)}^{\geq 0}|}{\nu\left(t^{\prime}, X\right)^{1 / 2}}\right) \\
& \leq C^{1 / 2} \kappa^{-1} N\left(t^{\prime}, t^{\prime \prime}, X\right)+C^{1 / 2} \kappa^{-1} \frac{\delta_{0}(t, X)-\delta_{0}\left(t^{\prime}, X\right)}{\nu\left(t^{\prime}, X\right)^{1 / 2}},
\end{aligned}
$$

which implies (2.3.3) provided that (2.3.5) holds. Finally, we may also assume that

$$
\nu(t, X) \leq \kappa^{2} \mu(t, X),
$$

otherwise we would have, using that $\delta_{0}\left(t^{\prime}, X\right) \leq \delta_{0}(t, X) \leq \delta_{0}\left(t^{\prime \prime}, X\right)$ and the convexity of $s \mapsto \sqrt{1+s^{2}}=\langle s\rangle$,

$$
R(t, X) \leq \kappa^{-1} \frac{\left\langle\delta_{0}(t, X)\right\rangle}{\Lambda^{1 / 2}} \leq \kappa^{-1} \frac{\left\langle\delta_{0}\left(t^{\prime}, X\right)\right\rangle}{\Lambda^{1 / 2}}+\kappa^{-1} \frac{\left\langle\delta_{0}\left(t^{\prime \prime}, X\right)\right\rangle}{\Lambda^{1 / 2}}
$$

and this implies, using $\nu\left(t^{\prime}, X\right), \nu\left(t^{\prime \prime}, X\right) \leq C \Lambda$ (see (2.1.17)),

$$
R(t, X) \leq C^{1 / 2} \kappa^{-1} \frac{\left\langle\delta_{0}\left(t^{\prime}, X\right)\right\rangle}{\nu\left(t^{\prime}, X\right)^{1 / 2}}+C^{1 / 2} \kappa^{-1} \frac{\left\langle\delta_{0}\left(t^{\prime \prime}, X\right)\right\rangle}{\nu\left(t^{\prime \prime}, X\right)^{1 / 2}}
$$

which gives (2.3.3) provided that (2.3.5) holds. On the other hand, we may assume that

$$
\max \left(\left\langle\delta_{0}(t, X)\right\rangle, \kappa^{1 / 2}\left|\Lambda^{1 / 2} q^{\prime}(t, X)\right|^{1 / 2}\right) \leq 2 \kappa \mu(t, X)^{1 / 2}
$$

BULLETIN DE LA SOCIÉTÉ MATHÉMATIQUE DE FRANCE 
Otherwise, we would have either

$$
\mu(t, X)^{1 / 2} \leq \frac{1}{2} \kappa^{-1}\left\langle\delta_{0}(t, X)\right\rangle \leq \frac{1}{2} \kappa^{-1} \nu(t, X)^{1 / 2} \underbrace{\leq}_{\text {from }(2.3 .7)} \frac{1}{2} \mu(t, X)^{1 / 2}
$$

which is impossible, or we would have

$$
\begin{gathered}
\mu(t, X)^{1 / 2} \leq \frac{1}{2} \kappa^{-1 / 2}\left|\Lambda^{1 / 2} q^{\prime}(t, X)\right|^{1 / 2} \overbrace{\leq}^{\text {from }} \frac{1}{2} \kappa^{-1 / 2} \nu(t, X)^{1 / 4} \mu(t, X)^{1 / 4} \\
\underbrace{\leq}_{\text {from (2.3.7) }} \frac{1}{2} \mu(t, X)^{1 / 2}, \quad \text { (which is also impossible). }
\end{gathered}
$$

The estimate (2.3.8) implies that, for $\kappa<1 / 16$,

$$
\begin{aligned}
& \Lambda\left|q^{\prime \prime}(t, X)\right|^{2} \underbrace{\leq}_{(2.1 .13)} \mu(t, X) \underbrace{\leq}_{(2.1 .13)}\left\langle\delta_{0}(t, X)\right\rangle^{2}+\left.\Lambda^{1 / 2} q^{\prime}(t, X)|+\Lambda| q^{\prime \prime}(t, X)\right|^{2} \\
& \underbrace{\leq}_{(2.3 .8)}\left(4 \kappa^{2}+4 \kappa\right) \mu(t, X)+\Lambda\left|q^{\prime \prime}(t, X)\right|^{2},
\end{aligned}
$$

$$
\Lambda\left|q^{\prime \prime}(t, X)\right|^{2} \leq \mu(t, X) \leq \frac{1}{1-8 \kappa} \Lambda\left|q^{\prime \prime}(t, X)\right|^{2} \leq 2 \Lambda\left|q^{\prime \prime}(t, X)\right|^{2}
$$

This implies that

$$
\begin{aligned}
R(t, X) \leq \Lambda^{-1 / 2} 2^{1 / 2} \Lambda^{1 / 2}\left|q^{\prime \prime}(t, X)\right| \frac{\left\langle\delta_{0}(t, X)\right\rangle}{\left(\left\langle\delta_{0}(t, X)\right\rangle^{2}+\Lambda\left|q^{\prime}(t, X)\right|^{2} \mu(t, X)^{-1}\right)^{1 / 2}} \\
\leq 2^{1 / 2}\left|q^{\prime \prime}(t, X)\right| .
\end{aligned}
$$

$\triangleright$ Rescaling the symbols. We sum-up our situation, changing the notations so that $X=0, t^{\prime}=t_{1}, t=t_{2}, t^{\prime \prime}=t_{3}, \nu_{1}=\nu\left(t^{\prime}, 0\right), \nu_{2}=\nu(t, 0), \nu_{3}=\nu\left(t^{\prime \prime}, 0\right), \delta_{j}=$ $\delta_{0}\left(t_{j}, 0\right), \mu_{j}=\mu\left(t_{j}, 0\right)$. The following conditions are satisfied:

$$
\left\{\begin{array}{r}
\left\langle\delta_{1}\right\rangle \leq \kappa \nu_{1}^{1 / 2}, \quad\left\langle\delta_{3}\right\rangle \leq \kappa \nu_{3}^{1 / 2}, \\
\nu_{2} \leq \kappa^{2} \nu_{1}, \quad \nu_{2} \leq \kappa^{2} \nu_{3}, \quad \nu_{2} \leq \kappa^{2} \mu_{2} \\
\left\langle\delta_{2}\right\rangle \\
\left.\Lambda \mid t_{2}, 0\right) \leq 2\left|q^{\prime \prime}\left(t_{2}, 0\right)\right| \frac{\left.q^{\prime \prime}\left(t_{2}, 0\right)\right|^{2} \leq \mu_{2} \leq 2 \Lambda\left|q^{\prime \prime}\left(t_{2}, 0\right)\right|^{2},}{\left\langle\delta_{2}\right\rangle+\left|q^{\prime}\left(t_{2}, 0\right)\right| /\left|q^{\prime \prime}\left(t_{2}, 0\right)\right|} \leq 2\left|q^{\prime \prime}\left(t_{2}, 0\right)\right|, \\
\kappa<1 / 16, \quad C_{0} \geq \kappa^{-1} C^{1 / 2},
\end{array}\right.
$$

where $\kappa>0$ is to be chosen later and $C$ depends only on a finite number of semi-norms of $q$. We define now the smooth functions $f_{1}, f_{2}$ defined on $\mathbb{R}^{2 n}$ by

$$
f_{1}(Y)=q\left(t_{1}, Y\right) \Lambda^{1 / 2} \mu_{1}^{-1 / 2}, \quad f_{2}(Y)=\nu_{1}^{1 / 2} q\left(t_{2}, Y\right)
$$

TOME $134-2006-\mathrm{N}^{\mathrm{O}} 4$ 
and we note (see (2.1.1)-(2.1.15)) that $\left\|f_{1}^{\prime \prime}\right\|_{L^{\infty}}$ and $\left\|f_{2}^{\prime \prime \prime}\right\|_{L^{\infty}}$ are bounded above by semi-norms of $q$; moreover the assumption (2.2.1) holds for that couple of functions, from (2.1.2).

LEMma 2.3.2. - We define

$$
\mu_{12}^{1 / 2}=\max \left(\left\langle\delta_{2}\right\rangle,\left|\nu_{1}^{1 / 2} q^{\prime}\left(t_{2}, 0\right)\right|^{1 / 2},\left|\nu_{1}^{1 / 2} q^{\prime \prime}\left(t_{2}, 0\right)\right|\right) .
$$

If $\max \left(\left\langle\delta_{2}\right\rangle, \kappa^{1 / 2}\left|\nu_{1}^{1 / 2} q^{\prime}\left(t_{2}, 0\right)\right|^{1 / 2}\right)>2 \kappa \mu_{12}^{1 / 2}$, then (2.3.3) is satisfied provided $C_{0} \geq 3 / \kappa$.

Proof. - We have either $\left|\nu_{1}^{1 / 2} q^{\prime \prime}\left(t_{2}, 0\right)\right| \leq \mu_{12}^{1 / 2} \leq \frac{1}{2} \kappa^{-1}\left\langle\delta_{2}\right\rangle$ implying

$$
\left|q^{\prime \prime}\left(t_{2}, 0\right)\right| \leq \frac{1}{2 \kappa} \frac{\left\langle\delta_{2}\right\rangle}{\nu_{1}^{1 / 2}} \leq \frac{1}{2 \kappa} \frac{\left\langle\delta_{1}\right\rangle}{\nu_{1}^{1 / 2}}+\frac{1}{2 \kappa} \frac{\delta_{2}-\delta_{1}}{\nu_{1}^{1 / 2}}
$$

which gives $(2.3 .3)$ ( using $R\left(t_{2}, 0\right) \leq 2\left|q^{\prime \prime}\left(t_{2}, 0\right)\right|$ in $(2.3 .11)$ ), provided $C_{0} \geq 1 / \kappa$, or we have

$$
\left|\nu_{1}^{1 / 2} q^{\prime \prime}\left(t_{2}, 0\right)\right| \leq \mu_{12}^{1 / 2}<\frac{1}{2} \kappa^{-1 / 2}\left|\nu_{1}^{1 / 2} q^{\prime}\left(t_{2}, 0\right)\right|^{1 / 2},
$$

implying $\frac{\left|q^{\prime \prime}\left(t_{2}, 0\right)\right|^{2}}{\left|q^{\prime}\left(t_{2}, 0\right)\right|} \leq \frac{1}{4 \kappa \nu_{1}^{1 / 2}}$ so that (using $R\left(t_{2}, 0\right) \leq 2\left|q^{\prime \prime}\left(t_{2}, 0\right)\right|^{2}\left\langle\delta_{2}\right\rangle /\left|q^{\prime}\left(t_{2}, 0\right)\right|$ in $(2.3 .11)$ ), we get $R\left(t_{2}, 0\right) \leq \frac{1}{2 \kappa} \frac{\left\langle\delta_{2}\right\rangle}{\nu_{1}^{1 / 2}}$, which gives similarly (2.3.3), provided $C_{0} \geq 1 /(2 \kappa)$.

A consequence of this lemma is that we may assume

$$
\begin{aligned}
& \max \left(\left\langle\delta_{2}\right\rangle, \kappa^{1 / 2}\left|\nu_{1}^{1 / 2} q^{\prime}\left(t_{2}, 0\right)\right|^{1 / 2}\right) \\
& \quad \leq 2 \kappa \mu_{12}^{1 / 2}=2 \kappa \max \left(\left\langle\delta_{2}\right\rangle,\left|\nu_{1}^{1 / 2} q^{\prime}\left(t_{2}, 0\right)\right|^{1 / 2},\left|\nu_{1}^{1 / 2} q^{\prime \prime}\left(t_{2}, 0\right)\right|\right),
\end{aligned}
$$

and since $\kappa<1 / 4$, we get

$(2.3 .14)$

$$
\mu_{12}^{1 / 2}=\left|\nu_{1}^{1 / 2} q^{\prime \prime}\left(t_{2}, 0\right)\right|, \quad \max \left(\left\langle\delta_{2}\right\rangle, \kappa^{1 / 2}\left|\nu_{1}^{1 / 2} q^{\prime}\left(t_{2}, 0\right)\right|^{1 / 2}\right) \leq 2 \kappa\left|\nu_{1}^{1 / 2} q^{\prime \prime}\left(t_{2}, 0\right)\right| .
$$

LEMMA 2.3.3. - The functions $f_{1}, f_{2}$ defined in (2.3.12) satisfy the assumptions (2.2.1-2-3-4-5-6) in the lemma 2.2.2.

Proof. - We have already checked (2.2.1). We know from Lemma 2.1.7 that, with a constant $C$ depending only on a finite number of semi-norms of $q$ (see $(2.1 .18))$,

$$
\left|f_{1}(0)=q\left(t_{1}, 0\right) \Lambda^{1 / 2} \mu_{1}^{-1 / 2}\right|^{1 / 2} \leq C \nu_{1}^{1 / 2},
$$

but we may assume here that $C \leq 1 / 2$ : if we had $\left|f_{1}(0)\right|>\nu_{1}^{1 / 2} / 2$, the function $f_{1}$ would be positive (resp.negative) on $B\left(0, r_{0} \nu_{1}^{1 / 2}\right.$ ), with some fixed $r_{0}>0$ and consequently we would have $\left|\delta_{1}\right| \geq r_{0} \nu_{1}^{1 / 2}$. But we know that $\left\langle\delta_{1}\right\rangle \leq \kappa \nu_{1}^{1 / 2}$, so we can choose a priori $\kappa$ small enough so that $\left|\delta_{1}\right| \geq r_{0} \nu_{1}^{1 / 2}$ does not occur. 
From (2.3.11), we have $\left\langle\delta_{1}\right\rangle \leq \kappa \nu_{1}^{1 / 2}$, the latter implying $f_{1}^{\prime}(0) \neq 0$ from $(2.1 .16)$ since $\kappa^{2}<3 / 4$ and more precisely

$$
\rho_{1}=\left|f_{1}^{\prime}(0)\right| \geq\left(1-\kappa^{2}\right)^{1 / 2} \nu_{1}^{1 / 2} \geq \nu_{1}^{1 / 2} / 2 .
$$

Moreover we have, from (2.1.18) and $\nu_{2} \leq \kappa^{2} \nu_{1}$ in (2.3.11),

$$
\max \left(\left|\nu_{1}^{1 / 2} q^{\prime}\left(t_{2}, 0\right)\right|^{1 / 2},\left|\nu_{1}^{1 / 2} q^{\prime \prime}\left(t_{2}, 0\right)\right|\right) \leq \mu_{12}^{1 / 2} \leq C_{1} \nu_{1}^{1 / 2},
$$

with a constant $C_{1}$ depending only on a finite number of semi-norms of $q$ and thus

$$
\max \left(\left|f_{2}^{\prime}(0)\right|^{1 / 2},\left|f_{2}^{\prime \prime}(0)\right|\right) \leq 2 C_{1} \rho_{1} .
$$

Moreover, we have from Lemma 2.1.7, $\Lambda^{1 / 2}\left|q\left(t_{2}, 0\right)\right| \mu_{2}^{-1 / 2} \leq C_{2} \nu_{2}$, so that with constants $C_{2}, C_{3}$ depending only on a finite number of semi-norms of $q$, using (2.3.8), we get

$$
\left|f_{2}(0)\right| \leq \nu_{1}^{1 / 2} C_{2} \nu_{2} \Lambda^{-1 / 2} \mu_{2}^{1 / 2} \leq \nu_{1}^{1 / 2} C_{3} \nu_{2} \leq C_{3} \kappa^{2} \nu_{1}^{3 / 2} .
$$

That property and (2.3.16-15) give (2.2.3) with $R_{0}=C \rho_{1}$, where $C$ depends only on a finite number of semi-norms of $q$. We have already seen that the constants occurring in (2.2.4) are bounded above by semi-norms of $q$ and that (2.2.5) holds. Let us now check (2.2.6). We already know that, from (2.3.14),

$$
\left|f_{2}^{\prime}(0)\right|^{1 / 2}=\left|\nu_{1}^{1 / 2} q^{\prime}\left(t_{2}, 0\right)\right|^{1 / 2} \leq 2 \kappa^{1 / 2}\left|\nu_{1}^{1 / 2} q^{\prime \prime}\left(t_{2}, 0\right)\right|=2 \kappa^{1 / 2}\left|f_{2}^{\prime \prime}(0)\right| .
$$

If we have $\left|\nu_{1}^{1 / 2} q\left(t_{2}, 0\right)\right| \geq \kappa^{1 / 2} \mu_{12}^{3 / 2}$ then for $|h| \leq \kappa^{1 / 3} \mu_{12}^{1 / 2}$, we get, using $\nu_{1} \lesssim \Lambda$ and Taylor's formula along with (2.3.13-14),

$$
\begin{aligned}
\left|\nu_{1}^{1 / 2} q\left(t_{2}, h\right)\right| \geq \kappa^{1 / 2} \mu_{12}^{3 / 2} & -4 \kappa^{4 / 3} \mu_{12}^{3 / 2}-\frac{1}{2} \kappa^{2 / 3} \mu_{12}^{3 / 2}-C \nu_{1}^{1 / 2} \Lambda^{-1 / 2} \kappa \mu_{12}^{3 / 2} \\
& =\mu_{12}^{3 / 2}\left(\kappa^{1 / 2}-4 \kappa^{4 / 3}-\frac{\kappa^{2 / 3}}{2}-C^{\prime} \kappa\right) \geq \mu_{12}^{3 / 2} \kappa^{1 / 2} / 2>0,
\end{aligned}
$$

provided $\kappa$ is small enough with respect to a constant depending only on a finite number of semi-norms of $q$; that inequality implies that the ball $B\left(0, \kappa^{1 / 3} \mu_{12}^{1 / 2}\right)$ is included in $\mathbb{X}_{+}\left(t_{2}\right)$ or in $\mathbb{X}_{-}\left(t_{2}\right)$ implying that $\left|\delta_{0}\left(t_{2}, 0\right)=\delta_{2}\right| \geq \kappa^{1 / 3} \mu_{12}^{1 / 2}$ which is incompatible with $(2.3 .14)$, provided $\kappa<2^{-3 / 2}$, since (2.3.14) implies $\left|\delta_{2}\right| \leq 2 \kappa \mu_{12}^{1 / 2}$. Eventually, we get

$$
\left|f_{2}(0)\right|^{1 / 3}=\left|\nu_{1}^{1 / 2} q\left(t_{2}, 0\right)\right|^{1 / 3} \leq \kappa^{1 / 6} \mu_{12}^{1 / 2}=\kappa^{1 / 6}\left|f_{2}^{\prime \prime}(0)\right|
$$

and with (2.3.18) we obtain (2.2.6) with

$$
\kappa_{2}=\kappa^{1 / 6} \text {. }
$$

The proof of Lemma 2.3.3 is complete.

TOME $134-2006-\mathrm{N}^{\mathrm{O}} 4$ 
$\triangleright$ End of the proof of Theorem 2.3.1. To apply Lemma 2.2.2, we have to suppose (2.2.7). In that case we get $\nu_{1}^{1 / 2}\left|q^{\prime \prime}\left(t_{2}, 0\right)_{-}\right|=\left|f_{2}^{\prime \prime}(0)_{-}\right| \leq C \kappa_{2} \rho_{2}=$ $C \kappa^{1 / 6} \nu_{1}^{1 / 2}\left|q^{\prime \prime}\left(t_{2}, 0\right)\right|$ i.e.,

$$
\left|q^{\prime \prime}\left(t_{2}, 0\right)_{-}\right| \leq C \kappa^{1 / 6}\left|q^{\prime \prime}\left(t_{2}, 0\right)\right| .
$$

If (2.2.7) is not satisfied, we obtain, according to (2.2.9), (2.3.19) and $\mu_{12}=$ $\nu_{1}^{1 / 2}\left|q^{\prime \prime}\left(t_{2}, 0\right)\right|$,

$$
\delta_{0}\left(t_{1}, 0\right)=\delta_{1} \leq-\kappa^{1 / 3} \nu_{1}^{1 / 2}\left|q^{\prime \prime}\left(t_{2}, 0\right)\right|,
$$

which gives $\frac{1}{3} R\left(t_{2}, 0\right) \leq\left|q^{\prime \prime}\left(t_{2}, 0\right)\right| \leq \kappa^{-1 / 3} \frac{\left|\delta_{1}\right|}{\nu_{1}^{1 / 2}}$ and (2.3.3) provided $C_{0} \geq$ $3 \kappa^{-1 / 3}$. If we introduce now the smooth functions $F_{1}, F_{2}$ defined on $\mathbb{R}^{2 n}$ by

$$
F_{1}(Y)=-q\left(t_{3}, Y\right) \Lambda^{1 / 2} \mu_{3}^{-1 / 2}, \quad F_{2}(Y)=-\nu_{3}^{1 / 2} q\left(t_{2}, Y\right),
$$

starting over our discussion, we see that (2.3.3) is satisfied, provided

$$
\kappa \leq \kappa_{0} \quad \text { and } \quad C_{0} \geq \gamma_{0} \kappa^{-1},
$$

where $\kappa_{0}, \gamma_{0}$ are positive constants depending only on the semi-norms of $q$, except in the case where we have (2.3.20) and

$$
\left|q^{\prime \prime}\left(t_{2}, 0\right)_{+}\right| \leq C \kappa^{1 / 6}\left|q^{\prime \prime}\left(t_{2}, 0\right)\right| \text {. }
$$

Naturally, since $\left|q^{\prime \prime}\left(t_{2}, 0\right)\right|=\left|q^{\prime \prime}\left(t_{2}, 0\right)_{+}\right|+\left|q^{\prime \prime}\left(t_{2}, 0\right)_{-}\right|$, the estimates (2.3.20-23) cannot be both true for a $\kappa$ small enough with respect to a constant depending on a finite number of semi-norms of $q$ and a non-vanishing $q^{\prime \prime}\left(t_{2}, 0\right)$ (that vanishing is prevented by the penultimate line in (2.3.11)). The proof of Theorem 2.3.1 is complete.

REMARK 2.3.4. - The readers may find our proof quite tedious, but referring them to the simpler remark following claim 2.2.1, we hope that they can find there some motivation to read the details of our argument, which is the rather natural quantitative statement following from that remark. On the other hand, Theorem 2.3.1 is analogous to one of the key argument provided by N. Dencker in [7] in which he proves, using our notations in the theorem,

$$
R(t, X) \lesssim N\left(t^{\prime}, t^{\prime \prime}, X\right)+\delta_{0}\left(t^{\prime \prime}, X\right)-\delta_{0}\left(t^{\prime}, X\right)
$$

which is weaker than our (2.3.3). In particular, $R$ (and $N$ ) looks like a symbol of order 0 (weight 1 ) whereas the right-hand-side of (2.3.24) contains the difference $\delta_{0}\left(t^{\prime \prime}, X\right)-\delta_{0}\left(t^{\prime}, X\right)$, which looks like a symbol of order $1 / 2$. Our theorem gives a stronger and in some sense more homogeneous version of N. Dencker's result, which will lead to improvements in the remainder's estimates. Also, we note the (inhomogeneous) estimate

$$
\Lambda^{-1 / 2} \mu(t, X)^{1 / 2} \nu(t, X)^{-1 / 2} \lesssim N\left(t^{\prime}, t^{\prime \prime}, X\right),
$$


which is in fact a consequence of our proof, but is not enough to handle the remainder's estimate below in our proof, and which will not be used: in fact (2.3.3) implies

$$
\begin{aligned}
& \Lambda^{-1 / 2} \mu^{1 / 2} \nu^{-1 / 2}=R\left\langle\delta_{0}\right\rangle^{-1} \\
& \lesssim \frac{N\left(t^{\prime}, t^{\prime \prime}, X\right)}{\left\langle\delta_{0}(t, X)\right\rangle}+\frac{\delta_{0}\left(t^{\prime \prime}, X\right)-\delta_{0}(t, X)}{\nu\left(t^{\prime \prime}, X\right)^{1 / 2}\left\langle\delta_{0}(t, X)\right\rangle}+\frac{\delta_{0}(t, X)-\delta_{0}\left(t^{\prime}, X\right)}{\nu\left(t^{\prime}, X\right)^{1 / 2}\left\langle\delta_{0}(t, X)\right\rangle} \\
& \quad \lesssim \frac{N\left(t^{\prime}, t^{\prime \prime}, X\right)}{\left\langle\delta_{0}(t, X)\right\rangle}+\frac{1}{\nu\left(t^{\prime \prime}, X\right)^{1 / 2}}+\frac{1}{\nu\left(t^{\prime}, X\right)^{1 / 2}} \lesssim N\left(t^{\prime}, t^{\prime \prime}, X\right) .
\end{aligned}
$$

2.4. Quasi-convexity. - A differentiable function $\psi$ of one variable is said to be quasi-convex on $\mathbb{R}$ if $\dot{\psi}(t)$ does not change sign from + to - for increasing $t$ (see [15]). In particular, a differentiable convex function is such that $\dot{\psi}(t)$ is increasing and is thus quasi-convex.

Definition 2.4.1. - Let $\sigma_{1}: \mathbb{R} \rightarrow \mathbb{R}$ be an increasing function, $C_{1}>0$ and let $\rho_{1}: \mathbb{R} \rightarrow \mathbb{R}_{+}$. We shall say that $\rho_{1}$ is quasi-convex with respect to $\left(C_{1}, \sigma_{1}\right)$ if for $t_{1}, t_{2}, t_{3} \in \mathbb{R}$,

$$
t_{1} \leq t_{2} \leq t_{3} \Longrightarrow \rho_{1}\left(t_{2}\right) \leq C_{1} \max \left(\rho_{1}\left(t_{1}\right), \rho_{1}\left(t_{3}\right)\right)+\sigma_{1}\left(t_{3}\right)-\sigma_{1}\left(t_{1}\right) .
$$

When $\sigma_{1}$ is a constant function and $C_{1}=1$, this is the definition of quasiconvexity.

LEMMA 2.4.2. - Let $\sigma_{1}: \mathbb{R} \rightarrow \mathbb{R}$ be an increasing function and let $\omega: \mathbb{R} \rightarrow$ $\mathbb{R}_{+}$. We define

$$
\rho_{1}(t)=\inf _{t^{\prime} \leq t \leq t^{\prime \prime}}\left(\omega\left(t^{\prime}\right)+\omega\left(t^{\prime \prime}\right)+\sigma_{1}\left(t^{\prime \prime}\right)-\sigma_{1}\left(t^{\prime}\right)\right) .
$$

Then the function $\rho_{1}$ is quasi-convex with respect to $\left(2, \sigma_{1}\right)$.

Proof. - We consider $t_{1} \leq t_{2} \leq t_{3}$ three real numbers. We have

$$
\begin{aligned}
& \rho_{1}\left(t_{2}\right)=\inf _{t^{\prime} \leq t_{2} \leq t^{\prime \prime}}\left(\omega\left(t^{\prime}\right)+\omega\left(t^{\prime \prime}\right)+\sigma_{1}\left(t^{\prime \prime}\right)-\sigma_{1}\left(t^{\prime}\right)\right) \\
& \leq \inf _{t^{\prime} \leq t_{1}, t_{3} \leq t^{\prime \prime}}\left(\omega\left(t^{\prime}\right)+\omega\left(t^{\prime \prime}\right)+\sigma_{1}\left(t^{\prime \prime}\right)-\sigma_{1}\left(t_{3}\right)+\sigma_{1}\left(t_{1}\right)-\sigma_{1}\left(t^{\prime}\right)\right) \\
& +\sigma_{1}\left(t_{3}\right)-\sigma_{1}\left(t_{1}\right) \\
& \begin{array}{r}
\leq \inf _{\substack{t^{\prime} \leq t_{1} \leq t_{1}^{\prime \prime} \\
t_{3}^{\prime} \leq t_{3} \leq t^{\prime \prime}}}\left(\omega\left(t^{\prime}\right)+\omega\left(t_{1}^{\prime \prime}\right)+\omega\left(t_{3}^{\prime}\right)+\omega\left(t^{\prime \prime}\right)+\sigma_{1}\left(t^{\prime \prime}\right)-\sigma_{1}\left(t_{3}^{\prime}\right)+\sigma_{1}\left(t_{1}^{\prime \prime}\right)-\sigma_{1}\left(t^{\prime}\right)\right) \\
+\sigma_{1}\left(t_{3}\right)-\sigma_{1}\left(t_{1}\right)
\end{array} \\
& =\rho_{1}\left(t_{1}\right)+\rho_{1}\left(t_{3}\right)+\sigma_{1}\left(t_{3}\right)-\sigma_{1}\left(t_{1}\right) \leq 2 \max \left(\rho_{1}\left(t_{1}\right), \rho_{1}\left(t_{3}\right)\right)+\sigma_{1}\left(t_{3}\right)-\sigma_{1}\left(t_{1}\right) \text {. }
\end{aligned}
$$

The following lemma is due to L. Hörmander [17].

TOME $134-2006-\mathrm{N}^{\mathrm{O}} 4$ 
LEMMA 2.4.3. - Let $\sigma_{1}: \mathbb{R} \rightarrow \mathbb{R}$ be an increasing function and let $\omega: \mathbb{R} \rightarrow$ $\mathbb{R}_{+}$. Let $T>0$ be given. We consider the function $\rho_{1}$ as defined in Lemma 2.4.2 and we define

$$
\Theta_{T}(t)=\sup _{-T \leq s \leq t}\left\{\sigma_{1}(s)-\sigma_{1}(t)+\frac{1}{2 T} \int_{s}^{t} \rho_{1}(r) d r-\rho_{1}(s)\right\} .
$$

Then we have

$$
2 T \partial_{t}\left(\Theta_{T}+\sigma_{1}\right) \geq \rho_{1}, \quad \text { and for }|t| \leq T, \quad\left|\Theta_{T}(t)\right| \leq \rho_{1}(t)
$$

Proof. - We have $\Theta_{T}(t) \geq-\rho_{1}(t)$, and

$$
\Theta_{T}(t)+\sigma_{1}(t)=\underbrace{\sup _{-T \leq s \leq t}\left\{\sigma_{1}(s)+\frac{1}{2 T} \int_{s}^{0} \rho_{1}(r) d r-\rho_{1}(s)\right\}}_{\text {increasing with } t}+\frac{1}{2 T} \int_{0}^{t} \rho_{1}(r) d r
$$

so that $\partial_{t}\left(\Theta_{T}+\sigma_{1}\right) \geq \frac{1}{2 T} \rho_{1}$. Moreover, from the proof of Lemma 2.4.2, we obtain for $s \leq r \leq t$ that $\rho_{1}(r) \leq \rho_{1}(s)+\rho_{1}(t)+\sigma_{1}(t)-\sigma_{1}(s)$ and thus

$$
\frac{1}{2 T} \int_{s}^{t} \rho_{1}(r) d r \leq \frac{1}{t-s} \int_{s}^{t} \rho_{1}(r) d r \leq \rho_{1}(s)+\rho_{1}(t)+\sigma_{1}(t)-\sigma_{1}(s)
$$

which gives $\Theta_{T}(t) \leq \rho_{1}(t)$, ending the proof of the lemma.

Definition 2.4.4. - For $T>0, X \in \mathbb{R}^{2 n},|t| \leq T$, we define

$$
\begin{aligned}
\omega(t, X) & =\frac{\left\langle\delta_{0}(t, X)\right\rangle}{\nu(t, X)^{1 / 2}}, \\
\sigma_{1}(t, X) & =\delta_{0}(t, X) \\
\eta(t, X) & =\int_{-T}^{t} \delta_{0}(s, X) \Lambda^{-1 / 2} d s+2 T,
\end{aligned}
$$

where $\delta_{0}, \nu$ are defined in (2.1.7),(2.1.16). For $T>0,(t, X) \in \mathbb{R} \times \mathbb{R}^{2 n}$, we define $\Theta(t, X)$ by the formula $(2.4 .3)$

$$
\Theta(t, X)=\sup _{-T \leq s \leq t}\left\{\sigma_{1}(s, X)-\sigma_{1}(t, X)+\frac{1}{2 T} \int_{s}^{t} \rho_{1}(r, X) d r-\rho_{1}(s, X)\right\},
$$

where $\rho_{1}$ is defined by (2.4.2). We define also

$$
m(t, X)=\delta_{0}(t, X)+\Theta(t, X)+T^{-1} \delta_{0}(t, X) \eta(t, X) .
$$

BULLETIN DE LA SOCIÉTÉ MATHÉMATIQUe DE FRANCE 
ThEOREM 2.4.5. - With the notations above for $\Theta, \rho_{1}, m$, with $R$ and $C_{0}$ defined in Theorem 2.3.1, we have for $T>0,|t| \leq T, X \in \mathbb{R}^{2 n}, \Lambda \geq 1$,

$$
\begin{gathered}
|\Theta(t, X)| \leq \rho_{1}(t, X) \leq 2 \frac{\left\langle\delta_{0}(t, X)\right\rangle}{\nu(t, X)^{1 / 2}}, \quad\left|\sigma_{1}(t, X)\right|=\left|\delta_{0}(t, X)\right| \\
C_{0}^{-1} R(t, X) \leq \rho_{1}(t, X) \leq 2 T \frac{\partial}{\partial t}\left(\Theta(t, X)+\sigma_{1}(t, X)\right) \\
0 \leq \eta(t, X) \leq 4 T, \quad \frac{d}{d t}\left(\delta_{0} \eta\right) \geq \delta_{0}^{2} \Lambda^{-1 / 2}, \quad\left|\eta_{X}^{\prime}(t, X)\right| \leq 4 T \Lambda^{-1 / 2} \\
T \frac{d}{d t} m \geq \frac{1}{2} \rho_{1}+\delta_{0}^{2} \Lambda^{-1 / 2} \geq \frac{1}{2 C_{0}} R+\delta_{0}^{2} \Lambda^{-1 / 2} \geq \frac{1}{2^{3 / 2} C_{0}}\left\langle\delta_{0}\right\rangle^{2} \Lambda^{-1 / 2}
\end{gathered}
$$

Proof. - It follows immediately from the previous results: the first estimate in (2.4.8) is (2.4.4), whereas the second is due to $\rho_{1} \leq 2 \omega$ which follows from (2.4.2). The equality in (2.4.8) follows from Definition 2.4.4. The first inequality in (2.4.9) is a consequence of (2.4.2) and (2.3.3) and the second is (2.4.4). The first two inequalities in (2.4.10) are a consequence of $\left|\delta_{0}(t, X)\right| \leq$ $\Lambda^{1 / 2}$ which follows from definition 2.1.4. The third inequality reads

$$
\frac{d}{d t}\left(\delta_{0} \eta\right)=\dot{\delta}_{0} \eta+\delta_{0} \dot{\eta} \geq \delta_{0} \dot{\eta}=\delta_{0}^{2} \Lambda^{-1 / 2}
$$

and the fourth inequality in (2.4.10) follows from (2.1.8). Let us check finally (2.4.11): since $m=\delta_{0}+\Theta+T^{-1} \delta_{0} \eta$, (2.4.4) and the already proven (2.4.10) imply $T \frac{d}{d t} m \geq \frac{1}{2} \rho_{1}+\delta_{0}^{2} \Lambda^{-1 / 2}$ and $(2.4 .9)$ (proven) gives

$$
\begin{gathered}
\frac{1}{2} \rho_{1}+\delta_{0}^{2} \Lambda^{-1 / 2} \geq \frac{1}{2 C_{0}} R+\delta_{0}^{2} \Lambda^{-1 / 2}=\frac{1}{2 C_{0}} \Lambda^{-1 / 2} \mu^{1 / 2} \nu^{-1 / 2}\left\langle\delta_{0}\right\rangle+\delta_{0}^{2} \Lambda^{-1 / 2} \\
\underbrace{\geq}_{\text {from }(2.1 .17)} \frac{1}{2 C_{0}} \Lambda^{-1 / 2}\left(2^{-1 / 2}\left\langle\delta_{0}\right\rangle+\delta_{0}^{2}\right) \geq \frac{1}{2^{3 / 2} C_{0}} \Lambda^{-1 / 2}\left\langle\delta_{0}\right\rangle^{2},
\end{gathered}
$$

completing the proof of Theorem 2.4.5.

\section{Energy estimates}

\subsection{Preliminaries}

Definition 3.1.1. - Let $T>0$ be given. With $m$ defined in (2.4.7), we define for $|t| \leq T$,

$$
M(t)=m(t, X)^{\mathrm{Wick}},
$$

where the Wick quantization is given by the definition A.1.1.

TOME $134-2006-\mathrm{N}^{\mathrm{O}} 4$ 
LEMMA 3.1.2. - With $T>0$ and $M$ given above, we have with $\rho_{1}$ given in (2.4.2), $R$ defined by (2.3.2), for $|t| \leq T, \Lambda \geq 1$,

$$
\begin{gathered}
\frac{d}{d t} M(t) \geq \frac{1}{2 T} \rho_{1}(t, X)^{\mathrm{Wick}}+\frac{1}{T}\left(\delta_{0}^{2}\right)^{\mathrm{Wick}} \Lambda^{-1 / 2} \\
\geq \frac{1}{2 C_{0} T} R^{\mathrm{Wick}}+T^{-1}\left(\delta_{0}^{2}\right)^{\mathrm{Wick}} \Lambda^{-1 / 2} \geq \frac{1}{2^{3 / 2} C_{0} T}\left(\left\langle\delta_{0}\right\rangle^{2}\right)^{\mathrm{Wick}} \Lambda^{-1 / 2} \\
|\Theta(t, X)| \leq \rho_{1}(t, X) \leq 2 \frac{\left\langle\delta_{0}(t, X)\right\rangle}{\nu(t, X)^{1 / 2}} \\
T^{-1}\left|\delta_{0}(t, X) \eta(t, X)\right| \leq 4\left|\delta_{0}(t, X)\right| \\
T^{-1}\left|\delta_{0 X}^{\prime}(t, X) \eta(t, X)\right|+T^{-1}\left|\delta_{0 X}(t, X) \eta_{X}^{\prime}(t, X)\right| \leq 12
\end{gathered}
$$

Proof. - The derivative in (3.1.2) is taken in the distribution sense, i.e., the first inequality in (3.1.2) means that (A.1.5) is satisfied with

$$
a(t, X)=m(t, X)-\frac{1}{2 T} \int_{-T}^{t} \rho_{1}(s, X) d s-\frac{1}{T} \Lambda^{-1 / 2} \int_{-T}^{t} \delta_{0}(s, X)^{2} d s .
$$

It follows in fact from (2.4.11). The other inequalities in (3.1.2) follow directly from (2.4.11) and the fact that the Wick quantization is positive (see (A.1.3)). The inequality (3.1.3) is (2.4.8) and (3.1.4) follows from (2.4.10) whereas (3.1.5) is a consequence of (2.1.8), (2.4.10) and Definition 2.1.4.

LEMma 3.1.3. - Using the definitions above and the notation (A.1.4), we have

$$
\begin{aligned}
\Theta(t, \cdot) & * \exp -2 \pi \Gamma \in S\left(\left\langle\delta_{0}(t, \cdot)\right\rangle \nu(t, \cdot)^{-1 / 2}, \Gamma\right), \\
\delta_{0}(t, \cdot) & * \exp -2 \pi \Gamma \in S\left(\left\langle\delta_{0}(t, \cdot)\right\rangle, \Gamma\right), \\
\delta_{0 X}^{\prime}(t, \cdot) & * \exp -2 \pi \Gamma \in S(1, \Gamma), \\
T^{-1} \eta(t, \cdot) & * \exp -2 \pi \Gamma \in S(1, \Gamma), \\
T^{-1} \eta(t, \cdot)_{X}^{\prime} & * \exp -2 \pi \Gamma \in S\left(\Lambda^{-1 / 2}, \Gamma\right),
\end{aligned}
$$

with semi-norms independent of $T \leq 1$ and of $t$ for $|t| \leq T$. According to the definition 1.3.1, the function $X \mapsto\left\langle\delta_{0}(t, X)\right\rangle$ is a $\Gamma$-weight.

Proof. - The last statement follows from (2.1.8). The inequalities ensuring (3.1.6 - 10) are then immediate consequences of the lemmas 3.1.2 and A.1.3.

3.2. Stationary estimates for the model cases. - Let $T>0$ be given and $Q(t)=q(t)^{w}$ given by (2.1.1-2). We define $M(t)$ according to (3.1.1). We consider

$$
\operatorname{Re}(Q(t) M(t))=\frac{1}{2} Q(t) M(t)+\frac{1}{2} M(t) Q(t)=P(t) .
$$

BULLETIN DE LA SOCIÉtÉ MATHÉMATIQUE DE FRANCE 
We have, omitting now the variable $t$ fixed throughout all this section 3.2 ,

$$
P=\operatorname{Re}\left[q^{w}\left(\delta_{0}\left(1+T^{-1} \eta\right)\right)^{\mathrm{Wick}}+q^{w} \Theta^{\mathrm{Wick}}\right] .
$$

[1]. Let us assume first that $q=\Lambda^{-1 / 2} \mu^{1 / 2} \nu^{1 / 2} \beta e_{0}$ with $\beta \in S\left(\nu^{1 / 2}, \nu^{-1} \Gamma\right), 1 \leq$ $e_{0} \in S\left(1, \nu^{-1} \Gamma\right)$ and $\delta_{0}=\beta$. Moreover, we assume $0 \leq T^{-1} \eta \leq 4, T^{-1}\left|\eta^{\prime}\right| \leq$ $4 \Lambda^{-1 / 2},|\Theta| \leq C\left\langle\delta_{0}\right\rangle \nu^{-1 / 2}$. Here $\Lambda, \mu, \nu$ are assumed to be positive constants such that $\Lambda \geq \mu \geq \nu \geq 1$

Then using the lemma A.1.5 with

$$
a_{1}=\beta e_{0}, \quad m_{1}=\langle\beta\rangle, \quad a_{2}=\left(1+T^{-1} \eta\right) e_{0}^{-1}, m_{2}=\nu^{-1 / 2},
$$

we get, with obvious notations,

$$
\left(\delta_{0} e_{0}\right)^{\mathrm{Wick}}\left(e_{0}^{-1}\left(1+T^{-1} \eta\right)\right)^{\mathrm{Wick}}=\left(\delta_{0}\left(1+T^{-1} \eta\right)\right)^{\mathrm{Wick}}+S\left(\left\langle\delta_{0}\right\rangle \nu^{-1 / 2}, \Gamma\right)^{w}
$$

and as a consequence from the proposition A.1.2(2), we obtain, with

$$
\beta_{0}=\beta e_{0}, \quad \eta_{0}=e_{0}^{-1}\left(1+T^{-1} \eta\right),
$$

the identity

$$
\left(\beta_{0}{ }^{w}+S\left(\nu^{-1 / 2}, \nu^{-1} \Gamma\right)^{w}\right) \eta_{0}^{\mathrm{Wick}}=\left(\delta_{0}\left(1+T^{-1} \eta\right)\right)^{\mathrm{Wick}}+S\left(\left\langle\delta_{0}\right\rangle \nu^{-1 / 2}, \Gamma\right)^{w}
$$

entailing

$$
\left(\delta_{0}\left(1+T^{-1} \eta\right)\right)^{\mathrm{Wick}}=\beta_{0}{ }^{w} \eta_{0}^{\mathrm{Wick}}+S\left(\left\langle\delta_{0}\right\rangle \nu^{-1 / 2}, \Gamma\right)^{w} .
$$

As a result, we have

$$
\begin{aligned}
& Q M=\Lambda^{-1 / 2} \mu^{1 / 2} \nu^{1 / 2} \beta_{0}^{w} \beta_{0}^{w} \eta_{0}^{\mathrm{Wick}}+\beta_{0}^{w} S(\overbrace{\Lambda^{-1 / 2} \mu^{1 / 2} \nu^{1 / 2}\left\langle\delta_{0}\right\rangle \nu^{-1 / 2}}^{=\Lambda^{-1 / 2} \mu^{1 / 2}\left\langle\delta_{0}\right\rangle}, \Gamma)^{w} \\
& +\beta_{0}^{w} S(\underbrace{\Lambda^{-1 / 2} \mu^{1 / 2} \nu^{1 / 2}\left\langle\delta_{0}\right\rangle \nu^{-1 / 2}}_{=\Lambda^{-1 / 2} \mu^{1 / 2}\left\langle\delta_{0}\right\rangle}, \Gamma)^{w} .
\end{aligned}
$$

This implies that, with $\gamma_{0}=1 / \sup e_{0}>0,\left(\right.$ so that $\left.1 \leq e_{0} \leq \gamma_{0}^{-1}\right)$

$$
\begin{aligned}
& 2 \operatorname{Re} Q M=2 \Lambda^{-1 / 2} \mu^{1 / 2} \nu^{1 / 2} \beta_{0}^{w} \eta_{0}^{\mathrm{Wick}} \beta_{0}^{w}+2 \operatorname{Re} \beta_{0}^{w} \Lambda^{-1 / 2} \mu^{1 / 2} \nu^{1 / 2} \overbrace{\left[\beta_{0}^{w}, \eta_{0}^{\mathrm{Wick}}\right]}^{\in S\left(\nu^{-1 / 2}, \Gamma\right)^{w}} . \\
& +\operatorname{Re} \beta_{0}^{w} S\left(\Lambda^{-1 / 2} \mu^{1 / 2}\left\langle\delta_{0}\right\rangle, \Gamma\right)^{w} \\
& =2 \Lambda^{-1 / 2} \mu^{1 / 2} \nu^{1 / 2} \beta_{0}^{w} \eta_{0}^{\mathrm{Wick}} \beta_{0}^{w}+\operatorname{Re} \beta_{0}^{w} S\left(\Lambda^{-1 / 2} \mu^{1 / 2}\left\langle\delta_{0}\right\rangle, \Gamma\right)^{w} \\
& 2 \Lambda^{-1 / 2} \mu^{1 / 2} \nu^{1 / 2} \beta_{0}^{w} \gamma_{0} \beta_{0}^{w}+\beta_{0}^{w} b_{0}^{w}+\bar{b}_{0}^{w} \beta_{0}^{w}, \\
& \begin{aligned}
\text { since } \eta_{0} \geq e_{0}^{-1} \\
\text { from } \eta \geq 0 \text { in }(2.4 .10)
\end{aligned}
\end{aligned}
$$


with $b_{0} \in S\left(\Lambda^{-1 / 2} \mu^{1 / 2}\left\langle\delta_{0}\right\rangle, \Gamma\right)$. With the notation $\lambda=\Lambda^{-1 / 2} \mu^{1 / 2} \nu^{1 / 2} \gamma_{0}$, we use the identity,

$\Lambda^{1 / 2} \mu^{1 / 2} \nu^{1 / 2} \beta_{0}^{w} \gamma_{0} \beta_{0}^{w}+\beta_{0}^{w} b_{0}^{w}+\bar{b}_{0}^{w} \beta_{0}^{w}=$

$$
\left(\lambda^{1 / 2} \beta_{0}^{w}+\lambda^{-1 / 2} \bar{b}_{0}^{w}\right)\left(\lambda^{1 / 2} \beta_{0}^{w}+\lambda^{-1 / 2} b_{0}^{w}\right)-\lambda^{-1} \bar{b}_{0}^{w} b_{0}^{w},
$$

so that from (3.2.4), we obtain the inequality

$$
2 \operatorname{Re} Q M+b_{1}^{w} \geq \Lambda^{-1 / 2} \mu^{1 / 2} \nu^{1 / 2} \gamma_{0} \beta_{0}^{w} \beta_{0}^{w} .
$$

with $b_{1}$ real valued in $S(\underbrace{\Lambda^{1 / 2} \mu^{-1 / 2} \nu^{-1 / 2} \Lambda^{-1} \mu\left\langle\delta_{0}\right\rangle^{2}}_{\Lambda^{-1 / 2} \mu^{1 / 2} \nu^{-1 / 2}\left\langle\delta_{0}\right\rangle^{2}}, \Gamma)$.

Using now (A.1.11), we get, with a "fixed" constant $C$, that

$b_{1}^{w} \leq C \Lambda^{-1 / 2} \mu^{1 / 2} \nu^{-1 / 2}\left(1+\beta^{2}\right)^{\text {Wick }}$

$$
\begin{aligned}
& =C \Lambda^{-1 / 2} \mu^{1 / 2} \nu^{-1 / 2} \operatorname{Id}+C \Lambda^{-1 / 2} \mu^{1 / 2} \nu^{-1 / 2}\left(\beta_{0}^{2} e_{0}^{-2}\right)^{\text {Wick }} \\
& \quad \leq C \Lambda^{-1 / 2} \mu^{1 / 2} \nu^{-1 / 2} \operatorname{Id}+C \Lambda^{-1 / 2} \mu^{1 / 2} \nu^{-1 / 2}\left(\beta_{0}^{2}\right)^{\text {Wick }},
\end{aligned}
$$

and since, from the proposition A.1.2(2), we have

$$
\left(\beta_{0}^{2}\right)^{\mathrm{Wick}}=\left(\beta_{0}^{2}\right)^{w}+S\left(1, \nu^{-1} \Gamma\right)^{w}=\beta_{0}^{w} \beta_{0}^{w}+S\left(1, \nu^{-1} \Gamma\right)^{w},
$$

the inequality (3.2.4) implies

$$
\begin{aligned}
& 2 \operatorname{Re} Q M+C \Lambda^{-1 / 2} \mu^{1 / 2} \nu^{-1 / 2} \operatorname{Id}+C \Lambda^{-1 / 2} \mu^{1 / 2} \nu^{-1 / 2} \beta_{0}{ }^{w} \beta_{0}{ }^{w}+ \\
& \quad+S\left(\Lambda^{-1 / 2} \mu^{1 / 2} \nu^{-1 / 2}, \nu^{-1} \Gamma\right)^{w} \geq 2 \operatorname{Re} Q M+b_{1}^{w} \geq \Lambda^{-1 / 2} \mu^{1 / 2} \nu^{1 / 2} \gamma_{0} \beta_{0}^{w} \beta_{0}^{w},
\end{aligned}
$$

so that

$$
\begin{aligned}
& \operatorname{Re} Q M+S\left(\Lambda^{-1 / 2} \mu^{1 / 2} \nu^{-1 / 2}, \Gamma\right)^{w} \\
& \quad \geq \beta_{0}^{w} \beta_{0}^{w}\left(\Lambda^{-1 / 2} \mu^{1 / 2} \nu^{1 / 2} \gamma_{0}-C^{\prime} \Lambda^{-1 / 2} \mu^{1 / 2} \nu^{-1 / 2}\right) .
\end{aligned}
$$

The rhs of (3.2.6) is nonnegative provided $\nu \geq C^{\prime} \gamma_{0}^{-1}$ and since $C^{\prime} \gamma_{0}^{-1}$ is a fixed constant, we may first suppose that this condition is satisfied; if it is not the case, we would have that $\nu$ is bounded above by a fixed constant and since $\nu \geq 1$, that would imply $q \in S\left(\Lambda^{-1 / 2} \mu^{1 / 2}, \Gamma\right)$ and $P \in S\left(\Lambda^{-1 / 2} \mu^{1 / 2}, \Gamma\right)^{w}$. In both cases, we get

$$
\operatorname{Re} Q M+S\left(\Lambda^{-1 / 2} \mu^{1 / 2} \nu^{-1 / 2}, \Gamma\right)^{w} \geq 0 .
$$

[2]. Let us assume now that $q \geq 0, q \in S\left(\Lambda^{-1 / 2} \mu^{1 / 2} \nu, \nu^{-1} \Gamma\right), \gamma_{0} \nu^{1 / 2} \leq \delta_{0} \leq$ $\gamma_{0}^{-1} \nu^{1 / 2}$ with a positive fixed constant $\gamma_{0}$. Moreover, we assume $0 \leq T^{-1} \eta \leq$ $4, T^{-1}\left|\eta^{\prime}\right| \leq 4 \Lambda^{-1 / 2},|\Theta(X)| \leq C, \Theta$ real-valued. Here $\Lambda, \mu, \nu$ are assumed to be positive constants such that $\Lambda \geq \mu \geq \nu \geq 1$.

We start over our discussion from the identity (3.2.2):

$$
P=\operatorname{Re}\left[q^{w}\left(\delta_{0}\left(1+T^{-1} \eta\right)+\Theta\right)^{\text {Wick }}\right] .
$$

BULlETIN DE LA SOCIÉtÉ MATHÉMATIQUE DE FRANCE 
We define

$$
a_{0}=\delta_{0}\left(1+T^{-1} \eta\right)
$$

and we note that $\gamma_{0} \nu^{1 / 2} \leq a_{0} \leq 5 \gamma_{0}^{-1} \nu^{1 / 2}$.

REMARK 3.2.1. - We may assume that $\nu^{1 / 2} \geq 2 C / \gamma_{0}$ which implies $C \leq$ $\frac{1}{2} \gamma_{0} \nu^{1 / 2}$ so that

$$
\frac{1}{2} \gamma_{0} \nu^{1 / 2} \leq a_{0}+\Theta \leq\left(5 \gamma_{0}^{-1}+\gamma_{0} / 2\right) \nu^{1 / 2}
$$

In fact if $\nu^{1 / 2}<2 C / \gamma_{0}$ we have $\left(\delta_{0}\left(1+T^{-1} \eta\right)+\Theta\right)^{\text {Wick }} \in S(1, \Gamma)^{w}$, $\Lambda^{1 / 2} \mu^{-1 / 2} q \in S(1, \Gamma)$ and $P \in S\left(\Lambda^{-1 / 2} \mu^{1 / 2}, \Gamma\right)^{w}$ so that (3.2.7) holds also in that case.

We have the identity

$$
q^{w}\left(\delta_{0}\left(1+T^{-1} \eta\right)\right)^{\mathrm{Wick}}=q^{w} a_{0}^{\mathrm{Wick}} \text { with }\left\{\begin{array}{l}
\gamma_{0} \nu^{1 / 2} \leq a_{0} \leq 5 \gamma_{0}^{-1} \nu^{1 / 2} \\
\left|a_{0}^{\prime}\right| \leq 14
\end{array}\right.
$$

The Weyl symbol of $\left(a_{0}+\Theta\right)^{\text {Wick}}$, which is

$$
a=\left(a_{0}+\Theta\right) * 2^{n} \exp -2 \pi \Gamma,
$$

belongs to $S_{1}\left(\nu^{1 / 2}, \nu^{-1} \Gamma\right)$ (see definition A.5.1): this follows from the lemma A.5.3 and (3.2.11) for $a_{0} * \exp -2 \pi \Gamma$ and is obvious for $\Theta * 2^{n} \exp -2 \pi \Gamma$ which belongs to $S(1, \Gamma)$. Moreover the estimates (3.2.10) imply that the symbol $a$ satisfies

$$
\frac{1}{2} \gamma_{0} \nu^{1 / 2} \leq a(X)=\int\left(a_{0}+\Theta\right)(X+Y) 2^{n} e^{-2 \pi|Y|^{2}} d Y \leq\left(5 \gamma_{0}^{-1}+C \gamma_{0} / 2\right) \nu^{1 / 2}
$$

As a result, the symbol $b=a^{1 / 2}$ belongs to $S_{1}\left(\nu^{1 / 4}, \nu^{-1} \Gamma\right)$ and $1 / b \in$ $S_{1}\left(\nu^{-1 / 4}, \nu^{-1} \Gamma\right)$ : we have

$$
2^{-1 / 2} \gamma_{0}^{1 / 2} \nu^{1 / 4} \leq|b| \leq\left(5 \gamma_{0}^{-1}+C \gamma_{0} / 2\right)^{1 / 2} \nu^{1 / 4}
$$

and moreover $a^{\prime}=a_{0}^{\prime} * 2^{n} \exp -2 \pi \Gamma+\Theta * 2^{n}(\exp -2 \pi \Gamma)^{\prime}$, so that, using

$$
\left|a^{\prime}\right| \leq 14+C\left\|2^{n}(\exp -2 \pi \Gamma)^{\prime}\right\|_{L^{1}\left(\mathbb{R}^{2 n}\right)}=C_{1},
$$

we get $2\left|b^{\prime}\right|=\left|a^{\prime}(X)\right| a(X)^{-1 / 2} \leq 2^{1 / 2} \gamma_{0}^{-1 / 2} \nu^{-1 / 4} C_{1}$, and the derivatives of $a^{1 / 2}$ of order $k \geq 2$ are a sum of terms of type

$$
a^{\frac{1}{2}-m} a^{\left(k_{1}\right)} \ldots a^{\left(k_{m}\right)}, \quad \text { with } k_{1}+\cdots+k_{m}=k, \text { all } k_{j} \geq 1,
$$

which can be estimated by $C \nu^{\frac{1}{4}-\frac{m}{2}} \leq C \nu^{-\frac{1}{4}}$ since $m \geq 1$. Similarly we obtain that $b^{-1} \in S_{1}\left(\nu^{-1 / 4}, \nu^{-1} \Gamma\right)$. From the lemma A.5.2, we have $b^{w} b^{w}=a^{w}+S\left(\nu^{-1 / 2}, \Gamma\right)^{w}=\left(a_{0}+\Theta\right)^{\mathrm{Wick}}+S\left(\nu^{-1 / 2}, \Gamma\right)^{w}$, which means

TOME $134-2006-\mathrm{N}^{\mathrm{O}} 4$ 
$\left(a_{0}+\Theta\right)^{\mathrm{Wick}}=b^{w} b^{w}+r_{0}^{w}, \quad r_{0} \in S\left(\nu^{-1 / 2}, \Gamma\right)$, real-valued. Using that $1 / b$ belongs to $S_{1}\left(\nu^{-1 / 4}, \nu^{-1} \Gamma\right)$, we write, using again the lemma A.5.2,

$$
\left(b+\frac{1}{2} b^{-1} r_{0}\right)^{w}\left(b+\frac{1}{2} b^{-1} r_{0}\right)^{w}=b^{w} b^{w}+r_{0}^{w}+S\left(\nu^{-1 / 4} \nu^{-1 / 2} \nu^{-1 / 4}, \Gamma\right)^{w},
$$

which gives,

$$
\left(a_{0}+\Theta\right)^{\mathrm{Wick}}=\left(b+\frac{1}{2} b^{-1} r_{0}\right)^{w}\left(b+\frac{1}{2} b^{-1} r_{0}\right)^{w}+S\left(\nu^{-1}, \Gamma\right)^{w} .
$$

Note that $b_{0}=b+\frac{1}{2} b^{-1} r_{0}$ belongs to $S_{1}\left(\nu^{1 / 4}, \nu^{-1} \Gamma\right)$ since it is true for $b$ and $b^{-1} r_{0} \in S\left(\nu^{-3 / 4}, \Gamma\right)$ : we get then

$$
2 \operatorname{Re}\left(q^{w}\left(a_{0}+\Theta\right)^{\mathrm{Wick}}\right)=2 b_{0}^{w} q^{w} b_{0}^{w}+\overbrace{[\underbrace{S\left(\Lambda^{-1 / 2} \mu^{1 / 2} \nu^{1 / 4} \nu^{-1 / 4}, \Gamma\right)}_{S\left(\Lambda^{-1 / 2} \mu^{1 / 2} \nu^{1 / 2} \nu^{-1 / 4}, \Gamma\right)}, b_{0}^{w}]}^{\left[q^{w}, b_{0}^{w}\right]}+\operatorname{Re}\left(q^{w} S\left(\nu^{-1}, \Gamma\right)^{w}\right)
$$

so that

$$
P=b_{0}^{w} q^{w} b_{0}^{w}+S\left(\Lambda^{-1 / 2} \mu^{1 / 2}, \Gamma\right)^{w} .
$$

Using now the Fefferman-Phong inequality ([8], Theorem 18.6.8 in [14]) for the nonnegative symbol $q$, we get $b_{0}^{w} q^{w} b_{0}^{w}=b_{0}^{w}\left(q^{w}+C \Lambda^{-1 / 2} \mu^{1 / 2} \nu^{-1}\right) b_{0}^{w}+$ $S\left(\Lambda^{-1 / 2} \mu^{1 / 2} \nu^{-1 / 2}, \Gamma\right)^{w} \geq S\left(\Lambda^{-1 / 2} \mu^{1 / 2} \nu^{-1 / 2}, \Gamma\right)^{w}$, so that, from (3.2.15) we get eventually

$$
\operatorname{Re}(Q M)+S\left(\Lambda^{-1 / 2} \mu^{1 / 2}, \Gamma\right)^{w} \geq 0
$$

3.3. Stationary estimates. - Let $T>0$ be given and $Q(t)=q(t)^{w}$ given by (2.1.1-2). We define $M(t)$ according to (3.1.1). We consider

$$
\operatorname{Re}(Q(t) M(t))=\frac{1}{2} Q(t) M(t)+\frac{1}{2} M(t) Q(t)=P(t) .
$$

We have, omitting now the variable $t$ fixed throughout all this section 3.3,

$$
P=\operatorname{Re}\left[q^{w}\left(\delta_{0}\left(1+T^{-1} \eta\right)\right)^{\mathrm{Wick}}+q^{w} \Theta^{\mathrm{Wick}}\right] .
$$

LEMma 3.3.1. - Let $p$ be the Weyl symbol of $P$ defined in (3.3.2) and $\widetilde{\Theta}=$ $\Theta * 2^{n} \exp -2 \pi \Gamma$, where $\Theta$ is defined in (2.4.6) (and satisfies (2.4.8)). Then we have

$$
\begin{aligned}
& p(t, X) \equiv p_{0}(t, X)=q(t, X)\left(\delta_{0}\left(1+T^{-1} \eta\right) * 2^{n} \exp -2 \pi \Gamma\right)+q(t, X) \widetilde{\Theta}(t, X), \\
& \text { modulo } S\left(\Lambda^{-1 / 2} \mu^{1 / 2} \nu^{-1 / 2}\left\langle\delta_{0}\right\rangle, \Gamma\right) .
\end{aligned}
$$


Proof. - Using the results of section 2.1, we know that the symbol $X \mapsto$ $q(t, X)$ belongs to the class

$$
S\left(\Lambda^{-1 / 2} \mu(t, X)^{1 / 2} \nu(t, X), \nu(t, X)^{-1} \Gamma\right)
$$

as shown in lemma 2.1.7. In fact from (2.1.18) we know that $q \in$ $S\left(\Lambda^{-1 / 2} \mu^{1 / 2} \nu, \nu^{-1} \Gamma\right)$, and from (3.1.3) and Lemma A.1.3, we obtain, using Theorem 18.5.5 in [14],

$$
q \sharp \widetilde{\Theta}=q \widetilde{\Theta}+\frac{1}{4 i \pi}\{q, \widetilde{\Theta}\}+S\left(\Lambda^{-1 / 2} \mu^{1 / 2} \nu^{-1 / 2}\left\langle\delta_{0}\right\rangle, \Gamma\right) .
$$

This implies that $\operatorname{Re}(q \sharp \widetilde{\Theta}) \in q \widetilde{\Theta}+S\left(\Lambda^{-1 / 2} \mu^{1 / 2} \nu^{-1 / 2}\left\langle\delta_{0}\right\rangle, \Gamma\right)$. On the other hand, we know that

$$
\begin{gathered}
\operatorname{Re}(q \sharp \overbrace{\left[\delta_{0}\left(1+T^{-1} \eta\right) * \exp -2 \pi \Gamma\right]}^{\omega}) \\
q \omega+\sum_{|\alpha|=|\beta|=2} c_{\alpha \beta} q^{(\alpha)} \omega^{(\beta)}+S\left(\Lambda^{-1 / 2} \mu^{1 / 2} \nu^{-1}, \Gamma\right)
\end{gathered}
$$

so that it is enough to concentrate our attention on the "products" $q^{\prime \prime} \omega^{\prime \prime}$. We have

$$
\left(\delta_{0}\left(1+T^{-1} \eta\right)\right)^{\prime \prime} * \exp -2 \pi \Gamma \in S(1, \Gamma)
$$

and since $q^{\prime \prime} \in S\left(\Lambda^{-1 / 2} \mu^{1 / 2}, \nu^{-1} \Gamma\right)$, we get a remainder in $S\left(\Lambda^{-1 / 2} \mu^{1 / 2}, \Gamma\right)$, which is fine as long as $\left\langle\delta_{0}\right\rangle \geq c \nu^{1 / 2}$. However when $\left\langle\delta_{0}\right\rangle \leq c \nu^{1 / 2}$, we know that, for a good choice of the fixed positive constant $c$, the function $\delta_{0}$ satisfies the estimates of $S\left(\nu^{1 / 2}, \nu^{-1} \Gamma\right)$, since it is the $\Gamma$-distance function to the set of (regular) zeroes of the function $q$ so that $q^{\prime \prime} \delta_{0}^{\prime \prime} \in S\left(\Lambda^{-1 / 2} \mu^{1 / 2} \nu^{-1 / 2}, \nu^{-1} \Gamma\right)$ which is what we are looking for. However, we are left with

$$
q^{\prime \prime}\left(\delta_{0} \eta * \exp -2 \pi \Gamma\right)^{\prime \prime} T^{-1} .
$$

Since we have $\left(\delta_{0} \eta\right)^{\prime \prime}=\delta_{0}^{\prime \prime} \eta+2 \delta_{0}^{\prime} \eta^{\prime}+\delta_{0} \eta^{\prime \prime}$ and $\left|\delta_{0}^{\prime \prime} \eta+2 \delta_{0}^{\prime} \eta^{\prime}\right| \leq C T\left(\nu^{-1 / 2}+\Lambda^{-1 / 2}\right)$, we have only to deal with the term

$$
\begin{aligned}
& \delta_{0} \eta^{\prime \prime} * \exp -2 \pi \Gamma=\int \delta_{0}(Y) \eta^{\prime \prime}(Y) \exp -2 \pi \Gamma(X-Y) d Y \\
&=-\int \underbrace{\delta_{0}^{\prime}(Y) \eta^{\prime}(Y)}_{\lesssim T \Lambda^{-1 / 2}} \exp -2 \pi \Gamma(X-Y) d Y \\
&-\int \underbrace{\delta_{0}(Y) \eta^{\prime}(Y)}_{\lesssim T \Lambda^{-1 / 2}\left\langle\delta_{0}\right\rangle} 4 \pi(X-Y) \exp -2 \pi \Gamma(X-Y) d Y .
\end{aligned}
$$

For future reference we summarize part of the previous discussion by the following result.

TOME $134-2006-\mathrm{N}^{\mathrm{O}} 4$ 
LEMMA 3.3.2. - With the notations above, we have

$$
\begin{gathered}
\left|\left(\delta_{0}\left(1+T^{-1} \eta\right) * \exp -2 \pi \Gamma\right)\right| \leq C\left\langle\delta_{0}\right\rangle, \quad\left|\left(\delta_{0}\left(1+T^{-1} \eta\right) * \exp -2 \pi \Gamma\right)^{\prime}\right| \leq C, \\
\left|\left(\delta_{0}\left(1+T^{-1} \eta\right) * \exp -2 \pi \Gamma\right)^{\prime \prime}\right| \leq C\left\langle\delta_{0}\right\rangle \nu^{-1 / 2}
\end{gathered}
$$

Proof. - Starting over the discussion, we have already seen that the result is true whenever $\left\langle\delta_{0}\right\rangle \gtrsim \nu^{1 / 2}$. Moreover when $\left\langle\delta_{0}\right\rangle \ll \nu^{1 / 2}$, we have seen that $\left|\delta_{0}^{\prime \prime}\right| \lesssim \nu^{-1 / 2}$ and $T^{-1}|\eta| \lesssim 1$; moreover we have already checked $\left|\eta^{\prime}\right| \lesssim T \Lambda^{-1 / 2}$ and $T^{-1}\left|\delta_{0}^{\prime} \eta^{\prime}\right| \lesssim \Lambda^{-1 / 2} \lesssim \nu^{-1 / 2}$ as well as $\left|\delta_{0} \eta^{\prime \prime} * \exp -2 \pi \Gamma\right| \lesssim \Lambda^{-1 / 2}\left\langle\delta_{0}\right\rangle \lesssim$ $\left\langle\delta_{0}\right\rangle \nu^{-1 / 2}$.

Eventually, using the lemma A.1.3, we get that the first integral above is in $S\left(T \Lambda^{-1 / 2}, \Gamma\right)$ whereas the second belongs to $S\left(T \Lambda^{-1 / 2}\left\langle\delta_{0}\right\rangle, \Gamma\right)$. Finally, it means that, up to terms in $S\left(\Lambda^{-1 / 2} \mu^{1 / 2} \nu^{-1 / 2}\left\langle\delta_{0}\right\rangle, \Gamma\right)$, the operator $P(t)$ has a Weyl symbol equal to the rhs of (3.3.3).

We shall use a partition of unity $1=\sum_{k} \chi_{k}^{2}$ related to the metric $\nu(t, X)^{-1} \Gamma$ and a sequence $\left(\psi_{k}\right)$ as in section 1.4. We have, omitting the variable $t$, with $p_{0}$ defined in (3.3.3),

$$
\begin{aligned}
p_{0}(X)=\sum_{k} \chi_{k}(X)^{2} q(X) \int & \delta_{0}(Y)\left(1+T^{-1} \eta(Y)\right) 2^{n} \exp -2 \pi \Gamma(X-Y) d Y \\
& +\sum_{k} \chi_{k}(X)^{2} q(X) \int \Theta(Y) 2^{n} \exp -2 \pi \Gamma(X-Y) d Y .
\end{aligned}
$$

Using the lemma A.1.6, we obtain, assuming $\delta_{0}=\delta_{0 k}, \Theta=\Theta_{k}, q=q_{k}$ on $U_{k}$

$$
\begin{aligned}
p_{0}=\sum_{k} \chi_{k}^{2} q_{k}( & \left.\delta_{0 k}\left(1+T^{-1} \eta\right) * 2^{n} \exp -2 \pi \Gamma\right) \\
& +\sum_{k} \chi_{k}^{2} q_{k}\left(\Theta_{k} * 2^{n} \exp -2 \pi \Gamma\right)+S\left(\Lambda^{-1 / 2} \mu^{1 / 2} \nu^{-\infty}, \Gamma\right) .
\end{aligned}
$$

LEMmA 3.3.3. - With $\widetilde{\Theta}_{k}=\Theta_{k} * 2^{n} \exp -2 \pi \Gamma, \quad d_{k}=\delta_{0 k}\left(1+T^{-1} \eta\right) *$ $2^{n} \exp -2 \pi \Gamma$ and $q_{k}, \chi_{k}$ defined above, we have

$$
\sum_{k} \chi_{k} \sharp q_{k} d_{k} \sharp \chi_{k}+\sum_{k} \chi_{k} \sharp q_{k} \widetilde{\Theta}_{k} \sharp \chi_{k}=p_{0}+S\left(\Lambda^{-1 / 2} \mu^{1 / 2} \nu^{-1 / 2}\left\langle\delta_{0}\right\rangle, \Gamma\right) .
$$

Proof. - We already know that $\left|d_{k}\right| \lesssim\left\langle\delta_{0}\right\rangle, \quad\left|d_{k}^{\prime}\right| \lesssim 1, \quad\left|d_{k}^{\prime \prime}\right| \lesssim\left\langle\delta_{0}\right\rangle \nu^{-1 / 2}$, so that

$$
\begin{array}{r}
\left|\left(q_{k} d_{k}\right)^{\prime \prime}=q_{k}^{\prime \prime} d_{k}+2 q_{k}^{\prime} d_{k}^{\prime}+q_{k} d_{k}^{\prime \prime}\right| \lesssim \Lambda^{-1 / 2} \mu^{1 / 2}\left(\left\langle\delta_{0}\right\rangle+\nu^{1 / 2}+\nu^{1 / 2}\left\langle\delta_{0}\right\rangle\right) \\
\lesssim \Lambda^{-1 / 2} \mu^{1 / 2} \nu^{1 / 2}\left\langle\delta_{0}\right\rangle .
\end{array}
$$

BULletin DE LA SOCIÉtÉ MATHÉMATIQUE DE FRANCE 
As a consequence, we get

$\sum_{k} \chi_{k} \sharp q_{k} d_{k} \sharp \chi_{k}$

$$
\begin{aligned}
& =\sum_{k}\left(\chi_{k} q_{k} d_{k}+\frac{1}{4 i \pi}\left\{\chi_{k}, q_{k} d_{k}\right\}+S\left(\nu^{-1}\left(\Lambda^{-1 / 2} \mu^{1 / 2}\left\langle\delta_{0}\right\rangle \nu^{1 / 2}\right), \Gamma\right)\right) \sharp \chi_{k} \\
& =\sum_{k}\left(\chi_{k} q_{k} d_{k}+\frac{1}{4 i \pi}\left\{\chi_{k}, q_{k} d_{k}\right\}\right) \sharp \chi_{k}+\sum_{k} S\left(\Lambda^{-1 / 2} \mu^{1 / 2}\left\langle\delta_{0}\right\rangle \nu^{-1 / 2}, \Gamma\right) \sharp \chi_{k} \\
& =\sum_{k}\left(\chi_{k} q_{k} d_{k}+\frac{1}{4 i \pi}\left\{\chi_{k}, q_{k} d_{k}\right\}\right) \chi_{k}+\frac{1}{4 i \pi} \sum_{k}\left\{\chi_{k} q_{k} d_{k}+\frac{1}{4 i \pi}\left\{\chi_{k}, q_{k} d_{k}\right\}, \chi_{k}\right\} \\
& +S\left(\Lambda^{-1 / 2} \mu^{1 / 2}\left\langle\delta_{0}\right\rangle \nu^{-1 / 2}, \Gamma\right)
\end{aligned}
$$

since $\left|\left(\chi_{k} q_{k} d_{k}\right)^{\prime \prime} \chi_{k}^{\prime \prime}\right| \lesssim \Lambda^{-1 / 2} \mu^{1 / 2}\left(\left\langle\delta_{0}\right\rangle+\nu^{1 / 2}+\nu\left\langle\delta_{0}\right\rangle \nu^{-1 / 2}\right) \nu^{-1} \lesssim\left\langle\delta_{0}\right\rangle \nu^{-1 / 2} \Lambda^{-1 / 2} \mu^{1 / 2}$. Using now that $\chi_{k} \sharp q_{k} d_{k} \sharp \chi_{k}$ is real-valued, we obtain

$$
\begin{aligned}
& \sum_{k} \chi_{k} \sharp q_{k} d_{k} \sharp \chi_{k}= \\
& \sum_{k} \chi_{k}^{2} q_{k} d_{k}-\frac{1}{16 \pi^{2}} \sum_{k}\left\{\left\{\chi_{k}, q_{k} d_{k}\right\}, \chi_{k}\right\}+S\left(\Lambda^{-1 / 2} \mu^{1 / 2}\left\langle\delta_{0}\right\rangle \nu^{-1 / 2}, \Gamma\right) .
\end{aligned}
$$

We note now that, using (3.3.6), we have

$$
\left\{\left\{\chi_{k}, q_{k} d_{k}\right\}, \chi_{k}\right\}=-H_{\chi_{k}}^{2}\left(q_{k} d_{k}\right) \in S\left(\Lambda^{-1 / 2} \mu^{1 / 2}\left\langle\delta_{0}\right\rangle \nu^{1 / 2} \nu^{-1}, \Gamma\right) .
$$

We examine now the term

$\chi_{k} \sharp q_{k} \widetilde{\Theta}_{k} \sharp \chi_{k}=$

$$
\left(\chi_{k} q_{k} \widetilde{\Theta}_{k}\right) \sharp \chi_{k}+\frac{1}{4 i \pi}\left\{\chi_{k}, q_{k} \widetilde{\Theta}_{k}\right\} \sharp \chi_{k}+S\left(\nu^{-1} \Lambda^{-1 / 2} \mu^{1 / 2} \nu\left\langle\delta_{0}\right\rangle \nu^{-1 / 2}, \Gamma\right) \sharp \chi_{k} .
$$

We have

$$
\begin{aligned}
& \operatorname{Re}\left(\chi_{k} q_{k} \widetilde{\Theta}_{k} \sharp \chi_{k}\right) \in \chi_{k}^{2} q_{k} \widetilde{\Theta}_{k}+S\left(\nu^{-1} \Lambda^{-1 / 2} \mu^{1 / 2} \nu\left\langle\delta_{0}\right\rangle \nu^{-1 / 2}, \Gamma\right), \\
& \frac{1}{4 i \pi}\left\{\chi_{k}, q_{k} \widetilde{\Theta}_{k}\right\} \in i \mathbb{R}+S\left(\nu^{-1 / 2} \Lambda^{-1 / 2} \mu^{1 / 2} \nu\left\langle\delta_{0}\right\rangle \nu^{-1 / 2}, \Gamma\right) .
\end{aligned}
$$

Since $\chi_{k} \sharp q_{k} \widetilde{\Theta}_{k} \sharp \chi_{k}$ is real-valued, we get

$$
\sum_{k} \chi_{k} \sharp q_{k} \widetilde{\Theta}_{k} \sharp \chi_{k}=\sum_{k} \chi_{k}^{2} q_{k} \widetilde{\Theta}_{k}+S\left(\Lambda^{-1 / 2} \mu^{1 / 2}\left\langle\delta_{0}\right\rangle \nu^{-1 / 2}, \Gamma\right) .
$$

Collecting the information (3.3.4), (3.3.7), (3.3.8) and (3.3.9) we obtain (3.3.5) and the lemma.

TOME $134-2006-\mathrm{N}^{\mathrm{O}} 4$ 
From this lemma and the lemma 3.3.1 we obtain that $(3.3 .10)$

$$
\operatorname{Re}(Q(t) M(t))=\sum_{k} \chi_{k}^{w}\left(q_{k} d_{k}+q_{k} \widetilde{\Theta}_{k}\right)^{w} \chi_{k}^{w}+S\left(\Lambda^{-1 / 2} \mu^{1 / 2}\left\langle\delta_{0}\right\rangle \nu^{-1 / 2}, \Gamma\right)^{w}
$$

Moreover the same arguments as above in Lemma 3.3.1 give also that

$$
\operatorname{Re}\left(q_{k}^{w} d_{k}^{w}+q_{k}^{w} \widetilde{\Theta}_{k}^{w}\right)=\left(q_{k} d_{k}+q_{k} \widetilde{\Theta}_{k}\right)^{w}+S\left(\Lambda^{-1 / 2} \mu^{1 / 2}\left\langle\delta_{0}\right\rangle \nu^{-1 / 2}, \Gamma\right)^{w}
$$

Proposition 3.3.4. - Let $T>0$ be given and $Q(t)=q(t)^{w}$ given by (2.1.1-2). We define $M(t)$ according to (3.1.1). Then, with a partition of unity $1=\sum_{k} \chi_{k}^{2}$ related to the metric $\nu(t, X)^{-1} \Gamma$ we have

$$
\begin{aligned}
\operatorname{Re}(Q(t) M(t))= & \sum_{k} \chi_{k}^{w} \operatorname{Re}\left(q_{k}^{w} d_{k}^{w}+q_{k}^{w} \widetilde{\Theta}_{k}^{w}\right) \chi_{k}^{w}+S\left(\Lambda^{-1 / 2} \mu^{1 / 2}\left\langle\delta_{0}\right\rangle \nu^{-1 / 2}, \Gamma\right)^{w} \\
& \operatorname{Re}(Q(t) M(t))+S\left(\Lambda^{-1 / 2} \mu^{1 / 2}\left\langle\delta_{0}\right\rangle \nu^{-1 / 2}, \Gamma\right)^{w} \geq 0 .
\end{aligned}
$$

Proof. — The equality (3.3.12) follows from (3.3.10-11). According to Lemma 2.1.9, we have to deal with four subsets of indices, $E_{ \pm}, E_{0}, E_{00}$. The classification in Definition 2.1.8 shows that section 3.2.[1] takes care of the cases $E_{0}$ and shows that, from (3.2.7),

$$
\text { for } k \in E_{0}, \quad \operatorname{Re}\left(q_{k}^{w} d_{k}^{w}+q_{k}^{w} \widetilde{\Theta}_{k}^{w}\right)+S\left(\Lambda^{-1 / 2} \mu^{1 / 2} \nu^{-1 / 2}, \Gamma\right)^{w} \geq 0 .
$$

Furthermore, the estimate (3.2.16) in section 3.2.[2] shows that

(3.3.15) for $k \in E_{ \pm}, \quad \operatorname{Re}\left(q_{k}^{w} d_{k}^{w}+q_{k}^{w} \widetilde{\Theta}_{k}^{w}\right)+S\left(\Lambda^{-1 / 2} \mu^{1 / 2} \nu^{-1 / 2}\left\langle\delta_{0}\right\rangle, \Gamma\right)^{w} \geq 0$.

Moreover if $k \in E_{00}$, the weight $\nu$ is bounded above and

$$
q_{k}^{w} d_{k}^{w}+q_{k}^{w} \widetilde{\Theta}_{k}^{w} \in S\left(\Lambda^{-1 / 2} \mu^{1 / 2} \nu^{-1 / 2}, \Gamma\right)^{w} .
$$

The equality (3.3.12) and (3.3.14-15-16) give (3.3.13).

\subsection{The multiplier method}

TheOREM 3.4.1. - Let $T>0$ be given and $Q(t)=q(t)^{w}$ given by (2.1.1-2). We define $M(t)$ according to (3.1.1). There exist $T_{0}>0$ and $c_{0}>0$ depending only on a finite number of $\gamma_{k}$ in (2.1.1) such that, for $0<T \leq T_{0}$, with $D(t, X)=\left\langle\delta_{0}(t, X)\right\rangle$, (D is Lipschitz continuous with Lipschitz constant 2, as $\delta_{0}$ in (2.1.8) and thus a $\Gamma$-weight),

$$
\frac{d}{d t} M(t)+2 \operatorname{Re}(Q(t) M(t)) \geq T^{-1}\left(D^{2}\right)^{\mathrm{Wick}} \Lambda^{-1 / 2} c_{0}
$$

BULLETIN DE LA SOCiÉTÉ MATHÉMATIQUe DE FRANCE 
Moreover we have with $m$ defined in $(2.4 .7), \widetilde{m}(t, \cdot)=m(t, \cdot) * 2^{n} \exp -2 \pi \Gamma$,

(3.4.2) $\quad M(t)=m(t, X)^{\mathrm{Wick}}=\widetilde{m}(t, X)^{w}$, with $\widetilde{m} \in S_{1}\left(D, D^{-2} \Gamma\right)+S(1, \Gamma)$.

$$
\begin{gathered}
m(t, X)=a(t, X)+b(t, X),|a / D|+\left|a_{X}^{\prime}\right|+|b| \text { bounded, } \dot{m} \geq 0, \\
a=\delta_{0}\left(1+T^{-1} \eta\right), \quad b=\widetilde{\Theta} .
\end{gathered}
$$

Proof. - From the estimate (3.1.2), we get, with a positive fixed constant $C_{0}$,

$$
\frac{d}{d t} M(t) \geq \frac{1}{2 C_{0} T}\left(\Lambda^{-1 / 2} \mu^{1 / 2} \nu^{-1 / 2}\left\langle\delta_{0}\right\rangle\right)^{\mathrm{Wick}}+T^{-1}\left(\delta_{0}^{2}\right)^{\mathrm{Wick}} \Lambda^{-1 / 2},
$$

and from (3.3.13) and Lemma A.1.4 we know that, with a fixed (nonnegative) constant $C_{1}$,

$$
2 \operatorname{Re}(Q(t) M(t))+C_{1}\left(\Lambda^{-1 / 2} \mu^{1 / 2} \nu^{-1 / 2}\left\langle\delta_{0}\right\rangle\right)^{\text {Wick }} \geq 0 .
$$

As a result we get, if $4 C_{1} C_{0} T \leq 1$ (we shall choose $T_{0}=\frac{1}{4 C_{0}\left(C_{1}+1\right)}$ ),

$\frac{d}{d t} M(t)+2 \operatorname{Re}(Q(t) M(t)) \geq \frac{1}{4 C_{0} T}\left(\Lambda^{-1 / 2} \mu^{1 / 2} \nu^{-1 / 2}\left\langle\delta_{0}\right\rangle\right)^{\mathrm{Wick}}+T^{-1}\left(\delta_{0}^{2}\right)^{\mathrm{Wick}} \Lambda^{-1 / 2}$.

Using $(2.1 .17)(\mu \geq \nu / 2)$, this gives

$$
\frac{d}{d t} M(t)+2 \operatorname{Re}(Q(t) M(t)) \geq T^{-1} \Lambda^{-1 / 2}\left(1+\delta_{0}^{2}\right)^{\mathrm{Wick}}\left(\frac{1}{2^{5 / 2} C_{0}+1}\right),
$$

which is the sought result.

\section{From semi-classical to local estimates}

4.1. From semi-classical to inhomogeneous estimates. - Let us consider a smooth real-valued function $f$ defined on $\mathbb{R} \times \mathbb{R}^{n} \times \mathbb{R}^{n}$, satisfiying (2.1.2) and such that, for all multi-indices $\alpha, \beta$,

$$
\sup _{\substack{t \in \mathbb{R} \\(x, \xi) \in \mathbb{R}^{2 n}}}\left|\left(\partial_{x}^{\alpha} \partial_{\xi}^{\beta} f\right)(t, x, \xi)\right|(1+|\xi|)^{-1+|\beta|}=C_{\alpha \beta}<\infty .
$$

Using a Littlewood-Paley decomposition, we have

$$
f(t, x, \xi)=\sum_{j \in \mathbb{N}} f(t, x, \xi) \varphi_{j}(\xi)^{2}, \operatorname{supp} \varphi_{0} \text { compact }
$$

for $j \geq 1, \operatorname{supp} \varphi_{j} \subset\left\{\xi \in \mathbb{R}^{n}, 2^{j-1} \leq|\xi| \leq 2^{j+1}\right\}, \sup _{j, \xi}\left|\partial_{\xi}^{\alpha} \varphi_{j}(\xi)\right| 2^{j|\alpha|}<\infty$.

We introduce also some smooth nonnegative compactly supported functions $\psi_{j}(\xi)$, satisfying the same estimates than $\varphi_{j}$ and supported in $2^{j-2} \leq|\xi| \leq 2^{j+2}$ for $j \geq 1$, identically 1 on the support of $\varphi_{j}$. For each $j \in \mathbb{N}$, we define the symbol

$$
q_{j}(t, x, \xi)=f(t, x, \xi) \psi_{j}(\xi)
$$

TOME $134-2006-\mathrm{N}^{\mathrm{O}} 4$ 
and we remark that (2.1.2) is satisfied for $q_{j}$ and the following estimates hold: $\left|\left(\partial_{x}^{\alpha} \partial_{\xi}^{\beta} q_{j}\right)\right| \leq C_{\alpha \beta}^{\prime} \Lambda_{j}^{1-|\beta|}$, with $\Lambda_{j}=2^{j}$. Note that the semi-norms of $q_{j}$ can be estimated from above independently of $j$. We can reformulate this by saying that

$$
\left.q_{j} \in S\left(\Lambda_{j}, \Lambda_{j}^{-1} \Gamma_{j}\right), \quad \text { with } \Gamma_{j}(t, \tau)=|t|^{2} \Lambda_{j}+|\tau|^{2} \Lambda_{j}^{-1} \text { (note that } \Gamma_{j}=\Gamma_{j}^{\sigma}\right) .
$$

LEMma 4.1.1. - There exists $T_{0}>0, c_{0}>0$, depending only on a finite number of semi-norms of $f$ such that, for each $j \in \mathbb{N}$, we can find $D_{j} a \Gamma_{j}$-uniformly Lipschitz continuous function with Lipschitz constant 2 , valued in $\left[1, \sqrt{2 \Lambda_{j}}\right]$, $a_{j}, b_{j}$ real-valued such that

$$
\sup _{\substack{j \in \mathbb{N},|t| \leq T_{0} \\ X \in \mathbb{R}^{2 n}}}\left(\left|\frac{a_{j}(t, X)}{D_{j}(t, X)}\right|+\left\|\nabla_{X} a_{j}(t, X)\right\|_{\Gamma_{j}}+\left|b_{j}(t, X)\right|\right)<\infty .
$$

Moreover we have with $m_{j}=a_{j}+b_{j}, \quad \tilde{m}_{j}(t, \cdot)=m_{j}(t, \cdot) * 2^{n} \exp -2 \pi \Gamma_{j}$, $Q_{j}(t)=q_{j}(t)^{w}$

$M_{j}(t)=m_{j}(t, X)^{\mathrm{Wick}\left(\Gamma_{j}\right)}=\widetilde{m}_{j}(t, X)^{w}$, with $\widetilde{m}_{j} \in S_{1}\left(D_{j}, D_{j}^{-2} \Gamma_{j}\right)+S\left(1, \Gamma_{j}\right)$,

(the Wick $\left(\Gamma_{j}\right)$ quantization is defined in definition A.1.7) the estimate

$$
\frac{d}{d t} M_{j}(t)+2 \operatorname{Re}\left(Q_{j}(t) M_{j}(t)\right) \geq T^{-1}\left(D_{j}^{2}\right)^{\mathrm{Wick}\left(\Gamma_{j}\right)} \Lambda_{j}^{-1 / 2} c_{0} .
$$

Proof. - It is a straightforward consequence of Definition A.1.7 and of Theorem 3.4.1: let us check this. Considering the linear symplectic mapping $L$ : $(t, \tau) \mapsto\left(\Lambda_{j}^{-1 / 2} t, \Lambda_{j}^{1 / 2} \tau\right)$, we see that the symbols $q_{j} \circ L$ belong uniformly to $S\left(\Lambda_{j}, \Lambda_{j}^{-1} \Gamma_{0}\right)$. Applying the theorem 3.4.1 to $q_{j} \circ L$, we find $D$ a $\Gamma_{0}$-uniformly Lipschitz continuous function $\geq 1, a, b$ real-valued such that

$$
\sup _{\substack{j \in \mathbb{N},|t| \leq T_{0} \\ X \in \mathbb{R}^{2 n}}}\left(\left|\frac{a(t, X)}{D(t, X)}\right|+\left\|\nabla_{X} a(t, X)\right\|_{\Gamma_{0}}+|b(t, X)|\right)<\infty,
$$

and so that, with $m=a+b, \quad \tilde{m}(t, \cdot)=m(t, \cdot) * 2^{n} \exp -2 \pi \Gamma_{0}, \quad Q(t)=$ $\left(q_{j}(t) \circ L\right)^{w}$,

$$
M(t)=m(t, X)^{\mathrm{Wick}}=\widetilde{m}(t, X)^{w}, \text { with } \tilde{m} \in S_{1}\left(D, D^{-2} \Gamma_{0}\right)+S\left(1, \Gamma_{0}\right),
$$

$$
\frac{d}{d t} M(t)+2 \operatorname{Re}(Q(t) M(t)) \geq T^{-1}\left(D^{2}\right)^{\mathrm{Wick}\left(\Gamma_{0}\right)} \Lambda_{j}^{-1 / 2} c_{0}
$$

BULLETIN DE LA SOCIÉTÉ MATHÉMATIQUe DE FRANCE 
Now we define the real-valued functions $a_{j}=a \circ L^{-1}, b_{j}=b \circ L^{-1}, D_{j}=D \circ L^{-1}$ and we have, since $\Gamma_{0}(S)=\Gamma_{j}(L S)$,

$$
\begin{aligned}
&\left|\frac{a_{j}(t, X)}{D_{j}(t, X)}\right|+\left\|\nabla_{X} a_{j}(t, X)\right\|_{\Gamma_{j}}+\left|b_{j}(t, X)\right| \\
&=\left|\frac{a\left(t, L^{-1} X\right)}{D\left(t, L^{-1} X\right)}\right|+\sup _{T \in \mathbb{R}^{2 n}} \frac{\left|a_{j}^{\prime}(t, X) \cdot T\right|}{\Gamma_{j}(T)^{1 / 2}}+\left|b\left(t, L^{-1} X\right)\right| \\
&=\left|\frac{a\left(t, L^{-1} X\right)}{D\left(t, L^{-1} X\right)}\right|+\sup _{T \in \mathbb{R}^{2 n}} \frac{\left|a^{\prime}(t, X) \cdot L^{-1} T\right|}{\Gamma_{j}(T)^{1 / 2}}+\left|b\left(t, L^{-1} X\right)\right| \\
&=\left|\frac{a\left(t, L^{-1} X\right)}{D\left(t, L^{-1} X\right)}\right|+\left\|a^{\prime}(t, X)\right\|_{\Gamma_{0}}+\left|b\left(t, L^{-1} X\right)\right|,
\end{aligned}
$$

so that (4.1.7) implies (4.1.4). Considering now $m_{j}=a_{j}+b_{j}$ and for a metaplectic $U$ in the fiber of the symplectic $L$ (see definition A.1.7), we have

$$
M_{j}(t)=m_{j}(t, X)^{\operatorname{Wick}\left(\Gamma_{j}\right)}=U\left(m_{j} \circ L\right)^{\operatorname{Wick}\left(\Gamma_{0}\right)} U^{*} .
$$

Thus we obtain

$$
\begin{aligned}
\frac{d}{d t} & M_{j}(t)+2 \operatorname{Re}\left(Q_{j}(t) M_{j}(t)\right) \\
\text { from (4.1.9.) } & =U \frac{d}{d t}\left(m_{j} \circ L\right)^{\operatorname{Wick}\left(\Gamma_{0}\right)} U^{*}+2 \operatorname{Re}\left(U U^{*} q_{j}(t)^{w} U\left(m_{j} \circ L\right)^{\operatorname{Wick}\left(\Gamma_{0}\right)} U^{*}\right) \\
& =U\left[\frac{d}{d t}\left(m_{j} \circ L\right)^{\operatorname{Wick}\left(\Gamma_{0}\right)}+2 \operatorname{Re}\left(U^{*} q_{j}(t)^{w} U\left(m_{j} \circ L\right)^{\operatorname{Wick}\left(\Gamma_{0}\right)}\right)\right] U^{*} \\
\text { using } \left.\begin{array}{c}
m=m_{j} \circ L \\
(q \circ)^{w}=U^{*} q^{w} U
\end{array}\right] & =U\left[\frac{d}{d t}(m)^{\operatorname{Wick}\left(\Gamma_{0}\right)}+2 \operatorname{Re}\left(\left(q_{j} \circ L\right)^{w}(m)^{\operatorname{Wick}\left(\Gamma_{0}\right)}\right)\right] U^{*} \\
\text { from (4.1.8) } & \geq U\left[T^{-1}\left(D^{2}\right)^{\operatorname{Wick}\left(\Gamma_{0}\right)} \Lambda_{j}^{-1 / 2} c_{0}\right] U^{*} \\
\text { from (A.1.16) } & =T^{-1} U U^{*}\left(D^{2} \circ L^{-1}\right)^{\operatorname{Wick}\left(\Gamma_{j}\right)} U U^{*} \Lambda_{j}^{-1 / 2} c_{0} \\
& =T^{-1}\left(D_{j}^{2}\right)^{\operatorname{Wick}\left(\Gamma_{j}\right)} \Lambda_{j}^{-1 / 2} c_{0},
\end{aligned}
$$

which is (4.1.6), completing the proof of the lemma.

We define now, with $\varphi_{j}$ given after (4.1.1), $M_{j}$ in (4.1.5)

$$
\mathcal{M}(t)=\sum_{j \in \mathbb{N}} \varphi_{j}^{w} \Lambda_{j}^{-1 / 2} M_{j}(t) \varphi_{j}^{w} .
$$

Lemma 4.1.2. - With $M_{j}$ defined in (4.1.5) and $\varphi_{j}, \psi_{j}$ as above,

$$
\begin{aligned}
& \sum_{j} \varphi_{j}^{w} M_{j}(t)\left(\left(1-\psi_{j}\right) f(t)\right)^{w} \varphi_{j}^{w} \in S\left(\langle\xi\rangle^{-\infty},\langle\xi\rangle|d x|^{2}+\langle\xi\rangle^{-1}|d \xi|^{2}\right)^{w}, \\
& \sum_{j} \varphi_{j}^{w} M_{j}(t) \varphi_{j}^{w}\left(\left(1-\psi_{j}\right) f(t)\right)^{w} \in S\left(\langle\xi\rangle^{-\infty},\langle\xi\rangle|d x|^{2}+\langle\xi\rangle^{-1}|d \xi|^{2}\right)^{w}
\end{aligned}
$$


Proof. - Since $\psi_{j} \equiv 1$ on the support of $\varphi_{j}$, we get that, uniformly with respect to $j$,

$$
\left(\left(1-\psi_{j}\right) f(t)\right)^{w} \varphi_{j}^{w} \in S\left(\langle\xi\rangle^{-\infty},|d x|^{2}+\langle\xi\rangle^{-2}|d \xi|^{2}\right)^{w} .
$$

Since $\widetilde{m_{j}} \in S\left(\Lambda_{j}^{1 / 2}, \Lambda_{j}|d x|^{2}+\Lambda_{j}^{-1}|d \xi|^{2}\right)$, we get that $\psi_{j} \widetilde{m_{j}} \in S\left(\langle\xi\rangle^{1 / 2},\langle\xi\rangle|d x|^{2}+\right.$ $\left.\langle\xi\rangle^{-1}|d \xi|^{2}\right)$, and consequently $\varphi_{j} \sharp \psi_{j} \widetilde{m_{j}} \in S\left(\langle\xi\rangle^{1 / 2},\langle\xi\rangle|d x|^{2}+\langle\xi\rangle^{-1}|d \xi|^{2}\right)$ so that

$$
\begin{aligned}
\varphi_{j} \sharp \psi_{j} \widetilde{m_{j} \sharp}\left(1-\psi_{j}\right) f(t) \sharp \varphi_{j} \in S\left(\langle\xi\rangle^{-\infty},\langle\xi\rangle|d x|^{2}+\langle\xi\rangle^{-1}|d \xi|^{2}\right) \\
\subset S\left(\langle\xi\rangle^{-\infty},|d x|^{2}+|d \xi|^{2}\right) .
\end{aligned}
$$

Moreover we have $\varphi_{j} \sharp\left(1-\psi_{j}\right) \widetilde{m}_{j} \in S\left(\Lambda_{j}^{-\infty}, \Lambda_{j}|d x|^{2}+\Lambda_{j}^{-1}|d \xi|^{2}\right) \subset S\left(\Lambda_{j}^{-\infty},|d x|^{2}+\right.$ $\left.|d \xi|^{2}\right)$ so that (4.1.13) implies

$$
\begin{aligned}
\varphi_{j} \sharp\left(1-\psi_{j}\right) \widetilde{m}_{j} \sharp\left(1-\psi_{j}\right) f(t) \sharp \varphi_{j} \in & S\left(\langle\xi\rangle^{-\infty},|d x|^{2}+|d \xi|^{2}\right) \\
& \subset S\left(\langle\xi\rangle^{-\infty},\langle\xi\rangle|d x|^{2}+\langle\xi\rangle^{-1}|d \xi|^{2}\right) .
\end{aligned}
$$

As a consequence, from (4.1.14) and (4.1.15) we get, uniformly in $j$, that

$$
\varphi_{j} \sharp \widetilde{m_{j} \sharp}\left(1-\psi_{j}\right) f(t) \sharp \varphi_{j} \in S\left(\langle\xi\rangle^{-\infty},\langle\xi\rangle|d x|^{2}+\langle\xi\rangle^{-1}|d \xi|^{2}\right) \text {. }
$$

Since $\varphi_{j}, \psi_{j}$ depend only on the variable $\xi$, the support condition implies $\varphi_{j}^{w} \psi_{j}^{w}=\varphi_{j}^{w}$ and we obtain that from (4.1.16)

$$
\begin{aligned}
\sum_{j} \varphi_{j} \sharp \widetilde{m}_{j} \sharp\left(1-\psi_{j}\right) f(t) \sharp \varphi_{j} \\
\quad=\sum_{j} \psi_{j} \sharp \varphi_{j} \sharp \widetilde{m_{j}} \sharp\left(1-\psi_{j}\right) f(t) \sharp \varphi_{j} \sharp \psi_{j} \in S\left(\langle\xi\rangle^{-\infty},\langle\xi\rangle|d x|^{2}+\langle\xi\rangle^{-1}|d \xi|^{2}\right),
\end{aligned}
$$

completing the proof of (4.1.11). The proof of (4.1.12) follows almost in the same way: we get as in (4.1.16) that

$$
\varphi_{j} \sharp \widetilde{m}_{j} \sharp \varphi_{j} \sharp\left(1-\psi_{j}\right) f(t) \in S\left(\langle\xi\rangle^{-\infty},\langle\xi\rangle|d x|^{2}+\langle\xi\rangle^{-1}|d \xi|^{2}\right) .
$$

Now with $\Phi_{j}=\varphi_{j} \sharp\left(1-\psi_{j}\right) f(t)$, we have $\Phi_{j} \in S\left(\langle\xi\rangle^{-\infty},|d x|^{2}+\langle\xi\rangle^{-2}|d \xi|^{2}\right)$ and from the formula (A.5.5) we have also $\left|\left(\partial_{x}^{\alpha} \partial_{\xi}^{\beta} \Phi_{j}\right)(x, \xi)\right| \leq C_{\alpha \beta N} 2^{j n}(1+\mid \xi-$ $\left.\operatorname{supp} \varphi_{j} \mid\right)^{-N}(1+|\xi|)$, so that

$$
\left|\left(\partial_{x}^{\alpha} \partial_{\xi}^{\beta} \Phi_{j}\right)(x, \xi)\right| \leq \begin{cases}C_{\alpha \beta N} 2^{j n} 2^{-j(N-1)} & \text { if }|\xi| \geq 2^{j+2} \\ C_{\alpha \beta N} 2^{j n} 2^{-j N} & \text { if } 2^{j-2}<|\xi|<2^{j+2} \\ C_{\alpha \beta N} 2^{j n} 2^{-j(N-1)} & \text { if }|\xi| \leq 2^{j-2}\end{cases}
$$

implying that $\sum_{j} \varphi_{j} \sharp \widetilde{m}_{j} \sharp \Phi_{j}$ belongs to $S\left(\langle\xi\rangle^{-\infty},|d x|^{2}+\langle\xi\rangle^{-2}|d \xi|^{2}\right)$. 
Lemma 4.1.3. - With $F(t)=f(t, x, \xi)^{w}, \mathcal{M}$ defined in (4.1.10), $M_{j}$ in (4.1.5)

$$
\begin{aligned}
\frac{d}{d t} \mathcal{M}(t)+2 \operatorname{Re}(\mathcal{M}(t) F(t)) & =\sum_{j} \Lambda_{j}^{-1 / 2} \varphi_{j}^{w}\left(\dot{M}_{j}(t)+2 \operatorname{Re}\left(M_{j}(t)\left(\psi_{j} f(t)\right)^{w}\right)\right) \varphi_{j}^{w} \\
& +\sum_{j} 2 \operatorname{Re}\left(\varphi_{j}^{w} M_{j}(t)\left[\varphi_{j}^{w},\left(\psi_{j} f(t)\right)^{w}\right] \Lambda_{j}^{-1 / 2}\right) \\
& +S\left(\langle\xi\rangle^{-\infty},\langle\xi\rangle|d x|^{2}+\langle\xi\rangle^{-1}|d \xi|^{2}\right)^{w}
\end{aligned}
$$

Proof. - We have

$$
\begin{aligned}
& \frac{d}{d t} \mathcal{M}(t)+2 \operatorname{Re}(\mathcal{M}(t) F(t)) \\
&=\sum_{j} \varphi_{j}^{w} \dot{M}_{j}(t) \Lambda_{j}^{-1 / 2} \varphi_{j}^{w}+2 \operatorname{Re}\left(\varphi_{j}^{w} M_{j}(t) \Lambda_{j}^{-1 / 2} \varphi_{j}^{w} F(t)\right) \\
&=\sum_{j} \varphi_{j}^{w} \dot{M}_{j}(t) \Lambda_{j}^{-1 / 2} \varphi_{j}^{w}+2 \operatorname{Re}\left(\varphi_{j}^{w} \Lambda_{j}^{-1 / 2} M_{j}(t) F(t) \varphi_{j}^{w}\right) \\
&+2 \operatorname{Re}\left(\varphi_{j}^{w} \Lambda_{j}^{-1 / 2} M_{j}(t)\left[\varphi_{j}^{w}, F(t)\right]\right) .
\end{aligned}
$$

On the other hand, we have

$$
\begin{aligned}
2 \operatorname{Re}\left(\varphi_{j}^{w} \Lambda_{j}^{-1 / 2} M_{j}(t) F(t) \varphi_{j}^{w}\right)=2 \operatorname{Re} & \left(\varphi_{j}^{w} \Lambda_{j}^{-1 / 2} M_{j}(t)\left(\psi_{j} f(t)\right)^{w} \varphi_{j}^{w}\right) \\
& +2 \operatorname{Re}\left(\varphi_{j}^{w} \Lambda_{j}^{-1 / 2} M_{j}(t)\left(\left(1-\psi_{j}\right) f(t)\right)^{w} \varphi_{j}^{w}\right)
\end{aligned}
$$

and since we have also

$$
\begin{aligned}
2 \operatorname{Re} & \left(\varphi_{j}^{w} \Lambda_{j}^{-1 / 2} M_{j}(t)\left[\varphi_{j}^{w}, F(t)\right]\right) \\
\quad & =2 \operatorname{Re}\left(\varphi_{j}^{w} \Lambda_{j}^{-1 / 2} M_{j}(t)\left[\varphi_{j}^{w},\left(\psi_{j} f(t)\right)^{w}\right]\right) \\
& +2 \operatorname{Re}\left(\varphi_{j}^{w} \Lambda_{j}^{-1 / 2} M_{j}(t)\left[\varphi_{j}^{w},\left(\left(1-\psi_{j}\right) f(t)\right)^{w}\right]\right),
\end{aligned}
$$

we get the result of the lemma from Lemma 4.1.2 and (4.1.17).

Lemma 4.1.4. - With the above notations, we have

$$
\sum_{j} \operatorname{Re}\left(\varphi_{j}^{w} \Lambda_{j}^{-1 / 2} M_{j}(t)\left[\varphi_{j}^{w},\left(\psi_{j} f(t)\right)^{w}\right]\right) \in S\left(\langle\xi\rangle^{-1},\langle\xi\rangle|d x|^{2}+\langle\xi\rangle^{-1}|d \xi|^{2}\right)^{w} .
$$

Proof. - The Weyl symbol of the bracket $\left[\varphi_{j}^{w},\left(\psi_{j} f(t)\right)^{w}\right]$ is $\frac{1}{2 i \pi}\left\{\varphi_{j}, \psi_{j} f(t)\right\}+$ $r_{j}, r_{j} \in S\left(\Lambda_{j}^{-1}, \Lambda_{j}^{-1} \Gamma_{j}\right)$ where $\left(r_{j}\right)$ is a confined sequence in $S\left(\langle\xi\rangle^{-1},|d x|^{2}+\right.$ $\left.\langle\xi\rangle^{-2}|d \xi|^{2}\right)$. As a consequence, we have

$$
\sum_{j} \varphi_{j}^{w} \Lambda_{j}^{-1 / 2} M_{j}(t) r_{j}^{w} \in S\left(\langle\xi\rangle^{-1},\langle\xi\rangle|d x|^{2}+\langle\xi\rangle^{-1}|d \xi|^{2}\right)^{w} .
$$

TOME $134-2006-\mathrm{N}^{\mathrm{O}} 4$ 
With $\Psi_{j}=-\frac{1}{2 \pi}\left\{\varphi_{j}, \psi_{j} f(t)\right\}$ (real-valued $\in S\left(1, \Lambda_{j}^{-1} \Gamma_{j}\right)$ ), we are left with $\sum_{j} \Lambda_{j}^{-1 / 2} \operatorname{Re}\left(\varphi_{j} \sharp \widetilde{m}_{j}(t) \sharp i \Psi_{j}\right)$ which belongs to $S\left(\langle\xi\rangle^{-1},\langle\xi\rangle|d x|^{2}+\langle\xi\rangle^{-1}|d \xi|^{2}\right)$.

Definition 4.1.5. - The symplectic metric $\Upsilon$ on $\mathbb{R}^{2 n}$ is defined as

$$
\Upsilon_{\xi}=\langle\xi\rangle|d x|^{2}+\langle\xi\rangle^{-1}|d \xi|^{2} .
$$

With $D_{j}$ given in lemma 4.1.1, we define

$$
d(t, x, \xi)=\sum_{j} \varphi_{j}(\xi)^{2} D_{j}(t, x, \xi) .
$$

LEMma 4.1.6. - The function $d(t, \cdot)$ is uniformly Lipschitz continuous for the metric $\Upsilon$ in the strongest sense, namely, there exists a positive fixed constant $C$ such that

$$
C^{-1}|d(t, x, \xi)-d(t, y, \eta)| \leq \min \left(\langle\xi\rangle^{1 / 2},\langle\eta\rangle^{1 / 2}\right)|x-y|+\frac{|\xi-\eta|}{\max \left(\langle\xi\rangle^{1 / 2},\langle\eta\rangle^{1 / 2}\right)} .
$$

Moreover it satisfies $d(t, x, \xi) \in\left[1,2\langle\xi\rangle^{1 / 2}\right]$. It is thus a weight for that metric $\Upsilon$.

Proof. - Since the $\varphi_{j}$ are nonnegative with $\sum_{j} \varphi_{j}^{2}=1$, we get from Lemma 4.1.1 that

$1=\sum_{j} \varphi_{j}^{2} \leq \sum_{j} \varphi_{j}^{2} D_{j}=d \leq \sum_{j} \varphi_{j}(\xi)^{2} \Lambda_{j}^{1 / 2} 2^{1 / 2} \leq \sum_{j} \varphi_{j}(\xi)^{2}\langle\xi\rangle^{1 / 2} 2=\langle\xi\rangle^{1 / 2} 2$.

Also, we have

$$
\begin{aligned}
& d(t, x, \xi)-d(t, y, \eta) \\
& \quad=\sum_{j} \varphi_{j}(\xi)^{2}\left(D_{j}(t, x, \xi)-D_{j}(t, y, \eta)\right)+\sum_{j} D_{j}(t, y, \eta)\left(\varphi_{j}(\xi)^{2}-\varphi_{j}(\eta)^{2}\right),
\end{aligned}
$$

so that, with $X=(x, \xi), Y=(y, \eta), \Gamma_{j}$ given in (4.1.3),

$$
\begin{aligned}
& |d(t, x, \xi)-d(t, y, \eta)| \\
& \quad \leq \sum_{j} \varphi_{j}(\xi)^{2} 2 \Gamma_{j}(X-Y)^{1 / 2}+\sum_{\substack{j, j \\
\varphi_{j}(\xi) \neq 0 \text { or } \varphi_{j}(\eta) \neq 0}} 2^{1 / 2} 2^{j / 2}|\xi-\eta| 2^{-j} C \\
& \quad \lesssim \sum_{j} \varphi_{j}(\xi)^{2} 4\left(\langle\xi\rangle^{1 / 2}|x-y|+\langle\xi\rangle^{-1 / 2}|\xi-\eta|\right)+|\xi-\eta| \sum_{\substack{j, \varphi_{j}(\xi) \neq 0 \text { or } \varphi_{j}(\eta) \neq 0}} 2^{-j / 2} \\
& \quad \lesssim\langle\xi\rangle^{1 / 2}|x-y|+\langle\xi\rangle^{-1 / 2}|\xi-\eta|+|\xi-\eta|\left(\langle\xi\rangle^{-1 / 2}+\langle\eta\rangle^{-1 / 2}\right) .
\end{aligned}
$$


We get thus, if $\langle\xi\rangle \sim\langle\eta\rangle$,

$$
|d(t, x, \xi)-d(t, y, \eta)| \lesssim\langle\xi\rangle^{1 / 2}|x-y|+\langle\xi\rangle^{-1 / 2}|\xi-\eta| .
$$

If $2^{j_{0}} \sim\langle\xi\rangle \ll\langle\eta\rangle \sim 2^{k_{0}}$, we have

$$
\begin{aligned}
|d(t, x, \xi)-d(t, y, \eta)| & \leq \sum_{j, \varphi_{j}(\xi) \neq 0} \varphi_{j}(\xi)^{2} 2^{(j+1) / 2}+\sum_{j, \varphi_{j}(\eta) \neq 0} \varphi_{j}(\eta)^{2} 2^{(j+1) / 2} \\
& \lesssim 2^{j_{0} / 2}+2^{k_{0} / 2} \sim 2^{k_{0} / 2} \sim|\eta-\xi| 2^{-k_{0} / 2} \sim\langle\eta\rangle^{-1 / 2}|\eta-\xi| .
\end{aligned}
$$

Eventually, (4.1.23) and (4.1.22) give (4.1.21), completing the proof of the lemma.

Note also that $\langle\xi\rangle$ is a $\Upsilon$-weight and is even such that

$$
\left|\langle\xi\rangle^{1 / 2}-\langle\eta\rangle^{1 / 2}\right| \leq \frac{|\xi-\eta|}{\langle\xi\rangle^{1 / 2}+\langle\eta\rangle^{1 / 2}} .
$$

Lemma 4.1.7. - With $F(t)=f(t, x, \xi)^{w}, \mathcal{M}$ defined in (4.1.10), $M_{j}$ in (4.1.5), the positive constant $c_{0}$ defined in lemma 4.1.1,

$\frac{d}{d t} \mathcal{M}(t)+2 \operatorname{Re}(\mathcal{M}(t) F(t)) \geq c_{0} T^{-1} \sum_{j} \varphi_{j}^{w}\left(\Lambda_{j}^{-1} D_{j}^{2}\right)^{\operatorname{Wick}\left(\Gamma_{j}\right)} \varphi_{j}^{w}+S\left(\langle\xi\rangle^{-1}, \Upsilon\right)^{w}$.

The operator $\mathcal{M}(t)$ has a Weyl symbol in the class $S_{1}\left(\langle\xi\rangle^{-1 / 2} d, d^{-2} \Upsilon\right)$. Moreover the selfadjoint operator $\mathcal{M}(t)$ satisfies, with a fixed constant $C$,

$$
\mathcal{M}(t) \mathcal{M}(t) \leq C^{2} \sum_{j} \varphi_{j}^{w}\left(\Lambda_{j}^{-1} D_{j}^{2}\right)^{\operatorname{Wick}\left(\Gamma_{j}\right)} \varphi_{j}^{w} .
$$

Proof. - The estimate (4.1.25) is a consequence of the lemmas 4.1.3, 4.1.4 and 4.1.1. From (4.1.10), we get that

$$
\mathcal{M}(t) \in \sum_{j} \varphi_{j}^{w} S_{1}\left(D_{j} \Lambda_{j}^{-1 / 2}, D_{j}^{-2} \Gamma_{j}\right)^{w} \varphi_{j}^{w} \subset S_{1}\left(d\langle\xi\rangle^{-1 / 2}, d^{-2} \Upsilon\right)^{w} .
$$

From the lemma 4.1.1 and the finite overlap of the $\varphi_{j}$, we get

$$
\begin{aligned}
& \|\mathcal{M}(t) u\|^{2} \lesssim \sum_{j} \Lambda_{j}^{-1}\left\|\varphi_{j}^{w} M_{j}(t) \varphi_{j}^{w} u\right\|^{2}=\sum_{j} \Lambda_{j}^{-1}\left\langle\varphi_{j}^{w} M_{j} \varphi_{j}^{w} u, \varphi_{j}^{w} M_{j} \varphi_{j}^{w} u\right\rangle= \\
& \sum_{j} \Lambda_{j}^{-1}\langle\varphi_{j}^{w} u, \underbrace{M_{j}\left(\varphi_{j}^{2}\right)^{w} M_{j}}_{\in S\left(D_{j}^{2}, \Gamma_{j}\right)^{w}} \varphi_{j}^{w} u\rangle \underbrace{\lesssim}_{\text {from lemma A.1.4 }} \sum_{j}\left\langle\varphi_{j}^{w} u,\left(\Lambda_{j}^{-1} D_{j}^{2}\right)^{\operatorname{Wick}\left(\Gamma_{j}\right)} \varphi_{j}^{w} u\right\rangle,
\end{aligned}
$$

which is (4.1.26). 
LEMMA 4.1.8. - Let a be a symbol in $S\left(\langle\xi\rangle^{-1}, \Upsilon\right)$. Then, with constants $C_{1}, C_{2}$ depending on a finite number of semi-norms of a, we have

$$
\left|\left\langle a^{w} u, u\right\rangle\right| \leq C_{1}\|u\|_{H^{-1 / 2}}^{2} \leq C_{2} \sum_{j}\left\langle\left(\Lambda_{j}^{-1} D_{j}^{2}\right)^{\operatorname{Wick}\left(\Gamma_{j}\right)} \varphi_{j}^{w} u, \varphi_{j}^{w} u\right\rangle .
$$

Proof. - We have, since $D_{j} \geq 1$ and the Wick quantizations are nonnegative

$$
\begin{aligned}
& \sum_{j}\left\langle\left(\Lambda_{j}^{-1} D_{j}^{2}\right)^{\operatorname{Wick}\left(\Gamma_{j}\right)} \varphi_{j}^{w} u, \varphi_{j}^{w} u\right\rangle \\
& \quad \geq \sum_{j}\left\langle\left(\Lambda_{j}^{-1}\right)^{\operatorname{Wick}\left(\Gamma_{j}\right)} \varphi_{j}^{w} u, \varphi_{j}^{w} u\right\rangle=\left\langle\left(\sum_{j} \Lambda_{j}^{-1} \varphi_{j}^{2}\right)^{w} u, u\right\rangle \sim\|u\|_{H^{-1 / 2}}^{2},
\end{aligned}
$$

where $H^{-1 / 2}$ is the standard Sobolev space of index $-1 / 2$. Now, it is a classical result that

$$
\left\langle a^{w} u, u\right\rangle=\langle\underbrace{\left(\langle\xi\rangle^{1 / 2}\right)^{w} a^{w}\left(\langle\xi\rangle^{1 / 2}\right)^{w}}_{\in S(1, \Upsilon)^{w} \subset \mathcal{L}\left(L^{2}\right)}\left(\langle\xi\rangle^{-1 / 2}\right)^{w} u,\left(\langle\xi\rangle^{-1 / 2}\right)^{w} u\rangle
$$

which implies that $\left|\left\langle a^{w} u, u\right\rangle\right| \lesssim\|u\|_{H^{-1 / 2}}^{2}$.

THEOREM 4.1.9. - Let $f(t, x, \xi)$ be a smooth real-valued function defined on $\mathbb{R} \times \mathbb{R}^{n} \times \mathbb{R}^{n}$, satisfiying (2.1.2) and (4.1.1). Let $f_{0}(t, x, \xi)$ be a smooth complexvalued function defined on $\mathbb{R} \times \mathbb{R}^{n} \times \mathbb{R}^{n}$, such that $\langle\xi\rangle f_{0}(t, x, \xi)$ satisfies (4.1.1). Then there exists $T_{0}>0, c_{0}>0$ depending on a finite number of seminorms of $f, f_{0}$, such that, for all $T \leq T_{0}$ and all $u \in C_{c}^{\infty}\left((-T, T) ; \mathcal{S}\left(\mathbb{R}^{n}\right)\right)$

$\left\|D_{t} u+i f(t, x, \xi)^{w} u+f_{0}(t, x, \xi)^{w} u\right\|_{L^{2}\left(\mathbb{R}^{n+1}\right)} \geq c_{0} T^{-1}\left(\int\|u(t)\|_{H^{-1 / 2}\left(\mathbb{R}^{n}\right)}^{2} d t\right)^{1 / 2}$

Proof

(i) We assume first that $f_{0} \equiv 0$. Using the lemmas 4.1.7-8, we get

(4.1.27) $2 \operatorname{Re}\left\langle D_{t} u+i f(t)^{w} u, i \mathcal{M}(t) u\right\rangle$

$$
\geq\left(c_{0} T^{-1}-C_{2}\right) \sum_{j}\left\langle\varphi_{j}^{w}\left(\Lambda_{j}^{-1} D_{j}^{2}\right)^{\operatorname{Wick}\left(\Gamma_{j}\right)} \varphi_{j}^{w} u, u\right\rangle,
$$

and from the estimate (4.1.26), provided that

$$
c_{0} /\left(2 C_{2}\right) \geq T
$$

we get

$$
\begin{aligned}
2\left\|D_{t} u+i f(t)^{w} u\right\|_{L^{2}\left(\mathbb{R}^{n+1}\right)}\left[\sum_{j}\left\langle\varphi_{j}^{w}\left(\Lambda_{j}^{-1} D_{j}^{2}\right)^{\mathrm{Wick}\left(\Gamma_{j}\right)} \varphi_{j}^{w} u, u\right\rangle\right]^{1 / 2} C \\
\geq \frac{c_{0}}{2 T} \sum_{j}\left\langle\varphi_{j}^{w}\left(\Lambda_{j}^{-1} D_{j}^{2}\right)^{\mathrm{Wick}\left(\Gamma_{j}\right)} \varphi_{j}^{w} u, u\right\rangle
\end{aligned}
$$

BULLETIN DE LA SOCiÉtÉ MATHÉMATIQUE DE FRANCE 
so that, with fixed positive constants $c_{1}, c_{2}$, using again the lemma 4.1.8

$$
\begin{aligned}
\left\|D_{t} u+i f(t)^{w} u\right\|_{L^{2}\left(\mathbb{R}^{n+1}\right)} \geq \frac{c_{1}}{T}\left[\sum_{j}\left\langle\varphi_{j}^{w}\left(\Lambda_{j}^{-1} D_{j}^{2}\right)^{\operatorname{Wick}\left(\Gamma_{j}\right)} \varphi_{j}^{w} u, u\right\rangle\right]^{1 / 2} & \\
& \geq \frac{c_{2}}{T}\left(\int\|u(t)\|_{H^{-1 / 2}\left(\mathbb{R}^{n}\right)}^{2} d t\right)^{1 / 2}
\end{aligned}
$$

which is our result. Let us check now the case $f_{0} \not \equiv 0$.

(ii) Let us assume that $\operatorname{Im}\left(f_{0}\right) \in S\left(\langle\xi\rangle^{-1},\langle\xi\rangle^{-1} \Upsilon\right)$. Going back to the computation in (4.1.27), with (4.1.28) fulfilled, we have

$$
\begin{aligned}
2 \operatorname{Re}\left\langle D_{t} u+i f(t)^{w}\right. & \left.+f_{0}(t)^{w} u, i \mathcal{M}(t) u\right\rangle \geq \frac{c_{0}}{2 T} \sum_{j}\left\langle\varphi_{j}^{w}\left(\Lambda_{j}^{-1} D_{j}^{2}\right)^{\operatorname{Wick}\left(\Gamma_{j}\right)} \varphi_{j}^{w} u, u\right\rangle \\
& +2 \operatorname{Re}\left\langle\operatorname{Re}\left(f_{0}(t)\right)^{w} u, i \mathcal{M}(t) u\right\rangle+2 \operatorname{Re}\left\langle\operatorname{Im}\left(f_{0}(t)\right)^{w} u, \mathcal{M}(t) u\right\rangle .
\end{aligned}
$$

From the identity $2 \operatorname{Re}\left\langle\operatorname{Re}\left(f_{0}(t)\right)^{w} u, i \mathcal{M}(t) u\right\rangle=\left\langle\left[\operatorname{Re}\left(f_{0}(t)\right)^{w}, i \mathcal{M}(t)\right] u, u\right\rangle$ and the fact that, from Theorem 18.5.5 in [14] we have

$$
\left[\operatorname{Re}\left(f_{0}(t)\right)^{w}, i \mathcal{M}(t)\right] \in S\left(\langle\xi\rangle^{-1 / 2} d d^{-1}\langle\xi\rangle^{-1 / 2}, \Upsilon\right)^{w}=S\left(\langle\xi\rangle^{-1}, \Upsilon\right)^{w}
$$

we can use the lemma 4.1 .8 to control this term by

$$
C \sum_{j}\left\langle\varphi_{j}^{w}\left(\Lambda_{j}^{-1} D_{j}^{2}\right)^{\operatorname{Wick}\left(\Gamma_{j}\right)} \varphi_{j}^{w} u, u\right\rangle .
$$

On the other hand, from our assumption on $\operatorname{Im} f_{0}$, we get that

$$
\mathcal{M}(t) \operatorname{Im}\left(f_{0}(t)\right)^{w} \in S\left(\langle\xi\rangle^{-1 / 2} d\langle\xi\rangle^{-1}, \Upsilon\right)^{w} \subset S\left(\langle\xi\rangle^{-1}, \Upsilon\right)^{w},
$$

which can be also controlled by $C \sum_{j}\left\langle\varphi_{j}^{w}\left(\Lambda_{j}^{-1} D_{j}^{2}\right)^{\mathrm{Wick}\left(\Gamma_{j}\right)} \varphi_{j}^{w} u, u\right\rangle$. Eventually, we obtain the result in that case too, for $T$ small enough.

(iii) We are left with the general case $\operatorname{Im}\left(f_{0}\right) \in S\left(1,\langle\xi\rangle^{-1} \Upsilon\right)$; we note that, with

(4.1.29) $\omega_{0}(t, x, \xi)=\int_{0}^{t} \operatorname{Im} f_{0}(s, x, \xi) d s, \quad\left(\right.$ which belongs to $\left.S\left(1,\langle\xi\rangle^{-1} \Upsilon\right)\right)$,

we have

$$
\begin{aligned}
& D_{t}+i f(t)^{w}+\left(\operatorname{Re} f_{0}(t)\right)^{w}+i\left(\operatorname{Im} f_{0}(t)\right)^{w} \\
& =\left(e^{\omega_{0}(t)}\right)^{w} D_{t}\left(e^{-\omega_{0}(t)}\right)^{w}+i f(t)^{w}+\left(\operatorname{Re} f_{0}(t)\right)^{w} \\
& =\left(e^{\omega_{0}(t)}\right)^{w}\left(D_{t}+i f(t)^{w}+\left(\operatorname{Re} f_{0}(t)\right)^{w}\right)\left(e^{-\omega_{0}(t)}\right)^{w}+\left(i f(t)-e^{\omega_{0}(t)} \sharp i f(t) \sharp e^{-\omega_{0}(t)}\right)^{w} \\
& +S\left(\langle\xi\rangle^{-1},\langle\xi\rangle^{-1} \Upsilon\right)^{w} .
\end{aligned}
$$

TOME $134-2006-\mathrm{N}^{\mathrm{O}} 4$ 
Noting that $e^{ \pm \omega_{0}}$ belongs to $S\left(1,\langle\xi\rangle^{-1} \Upsilon\right)$, we compute

$$
\begin{aligned}
e^{\omega_{0}} \sharp i f \sharp e^{-\omega_{0}} & =\left(e^{\omega_{0}} \text { if }+\frac{1}{4 i \pi}\left\{e^{\omega_{0}}, i f\right\}\right) \sharp e^{-\omega_{0}}+S\left(\langle\xi\rangle^{-1},\langle\xi\rangle^{-1} \Upsilon\right) \\
& =i f+\frac{1}{4 i \pi}\left\{e^{\omega_{0}} i f, e^{-\omega_{0}}\right\}+\frac{1}{4 i \pi}\left\{e^{\omega_{0}}, i f\right\} e^{-\omega_{0}}+S\left(\langle\xi\rangle^{-1},\langle\xi\rangle^{-1} \Upsilon\right) \\
& =i f+\frac{1}{2 \pi}\left\{\omega_{0}, f\right\}+S\left(\langle\xi\rangle^{-1},\langle\xi\rangle^{-1} \Upsilon\right) .
\end{aligned}
$$

We obtain

(4.1.30)

$$
\begin{aligned}
L= & D_{t}+i f(t)^{w}+f_{0}(t)^{w} \\
=\left(e^{\omega_{0}(t)}\right)^{w}\left(D_{t}+i f(t)^{w}+\left(\operatorname{Re} f_{0}(t)+\frac{1}{2 \pi}\left\{f, \omega_{0}\right\}\right)^{w}\right)\left(e^{-\omega_{0}(t)}\right)^{w} & \quad+S\left(\langle\xi\rangle^{-1},\langle\xi\rangle^{-1} \Upsilon\right)^{w},
\end{aligned}
$$

and analogously

$$
\begin{aligned}
L_{0} & =D_{t}+i f(t)^{w}+\left(\operatorname{Re} f_{0}(t)+\frac{1}{2 \pi}\left\{f, \omega_{0}\right\}\right)^{w}+S\left(\langle\xi\rangle^{-1},\langle\xi\rangle^{-1} \Upsilon\right)^{w} \\
& =\left(e^{-\omega_{0}(t)}\right)^{w} L\left(e^{\omega_{0}(t)}\right)^{w}
\end{aligned}
$$

Using now the fact that the symbol $\operatorname{Re} f_{0}(t)+\frac{1}{2 \pi}\left\{f, \omega_{0}\right\}$ is real-valued in $S\left(1,\langle\xi\rangle^{-1} \Upsilon\right)$, we can use (ii) to prove the estimate in the theorem for the operator

$$
L_{0}=D_{t}+i f(t)^{w}+\left(\operatorname{Re} f_{0}(t)+\frac{1}{2 \pi}\left\{f, \omega_{0}\right\}\right)^{w}+S\left(\langle\xi\rangle^{-1},\langle\xi\rangle^{-1} \Upsilon\right)^{w} .
$$

We note also that $e^{\omega_{0}} \sharp e^{-\omega_{0}}=1+t^{2} S\left(\langle\xi\rangle^{-2},\langle\xi\rangle^{-1} \Upsilon\right)$ so that, for $|t|$ small enough,

$$
\left\{\begin{array}{c}
\text { the operators }\left(e^{ \pm \omega_{0}}\right)^{w} \text { are invertible in } L^{2}\left(\mathbb{R}^{n}\right) \text { and } \\
\text { their inverses are pseudodifferential operators in } S\left(1,\langle\xi\rangle^{-1} \Upsilon\right)^{w} .
\end{array}\right.
$$

From the previous identity and (ii), we get for $u \in C_{\mathrm{c}}^{\infty}\left((-T, T),>\mathcal{S}\left(\mathbb{R}^{n}\right)\right)$

$$
\int\left\|\left(e^{-\omega_{0}(t)}\right)^{w} L\left(e^{\omega_{0}(t)}\right)^{w} u(t)\right\|_{L^{2}\left(\mathbb{R}^{n}\right)}^{2} d t \geq \frac{c_{0}^{2}}{T^{2}} \int\|u(t)\|_{H^{-1 / 2}\left(\mathbb{R}^{n}\right)}^{2} d t .
$$

Applying this to

$$
u(t)=\left(\left(e^{\omega_{0}(t)}\right)^{w}\right)^{-1} v(t)
$$

we obtain

$$
\int\left\|\left(e^{-\omega_{0}(t)}\right)^{w} L v(t)\right\|_{L^{2}\left(\mathbb{R}^{n}\right)}^{2} d t \geq \frac{c_{0}^{2}}{T^{2}} \int\left\|\left(\left(e^{\omega_{0}(t)}\right)^{w}\right)^{-1} v(t)\right\|_{H^{-1 / 2}\left(\mathbb{R}^{n}\right)}^{2} d t
$$

BULLETIN DE LA SOCIÉTÉ MATHÉMATIQUE DE FRANCE 
We have

$$
\begin{aligned}
\left\|\left(\left(e^{\omega_{0}(t)}\right)^{w}\right)^{-1} v(t)\right\|_{H^{-1 / 2}\left(\mathbb{R}^{n}\right)}^{2} & =\left\|\left(\langle\xi\rangle^{-1 / 2}\right)^{w}\left(\left(e^{\omega_{0}(t)}\right)^{w}\right)^{-1}\left(\langle\xi\rangle^{1 / 2}\right)^{w}\left(\langle\xi\rangle^{-1 / 2}\right)^{w} v(t)\right\|_{L^{2}\left(\mathbb{R}^{n}\right)}^{2} .
\end{aligned}
$$

Now the operator $\left(\langle\xi\rangle^{-1 / 2}\right)^{w}\left(\left(e^{\omega_{0}(t)}\right)^{w}\right)^{-1}\left(\langle\xi\rangle^{1 / 2}\right)^{w}$ is invertible with inverse

$$
\Omega(t)=\left(\langle\xi\rangle^{-1 / 2}\right)^{w}\left(e^{\omega_{0}(t)}\right)^{w}\left(\langle\xi\rangle^{1 / 2}\right)^{w}
$$

which is a bounded operator on $L^{2}\left(\mathbb{R}^{n}\right)$ so that

$$
\|v\|_{L^{2}}=\left\|\Omega \Omega^{-1} v\right\|_{L^{2}} \leq\|\Omega\|_{\mathcal{L}\left(L^{2}\right)}\left\|\Omega^{-1} v\right\|_{L^{2}} .
$$

As a result, from the inequality (4.1.34), we get

$$
\begin{aligned}
& \int\left\|\left(e^{-\omega_{0}(t)}\right)^{w} L v(t)\right\|_{L^{2}\left(\mathbb{R}^{n}\right)}^{2} d t \geq \frac{c_{0}^{2}}{T^{2}} \int\left\|\Omega(t)^{-1}\left(\langle\xi\rangle^{-1 / 2}\right)^{w} v(t)\right\|_{L^{2}\left(\mathbb{R}^{n}\right)}^{2} d t \\
& \quad \geq \frac{c_{0}^{2}}{T^{2}} \int\left\|\left(\langle\xi\rangle^{-1 / 2}\right)^{w} v(t)\right\|_{L^{2}\left(\mathbb{R}^{n}\right)}^{2} \frac{1}{\|\Omega(t)\|^{2}} d t \geq \frac{c_{1}^{2}}{T^{2}} \int\|v(t)\|_{H^{-1 / 2}\left(\mathbb{R}^{n}\right)}^{2} d t
\end{aligned}
$$

which is the result. The proof of Theorem 4.1.9 is complete.

Comment 4.1.10. - Although Theorem 4.1.9 is providing a solvability result with loss of $3 / 2$ derivatives for the evolution equation

$$
\partial_{t}+f(t, x, \xi)^{w}+f_{0}(t, x, \xi)^{w},
$$

where $f, f_{0}$ are satisfying the assumptions of this theorem, the statement does not seem quite sufficient to handle operators with homogeneous symbols for two reasons. The first one is that the reduction of homogeneous symbols in the cotangent bundle of a manifold will lead to a model operator like the one above, but only at the cost of some microlocalization in the cotangent bundle. We need thus to get a microlocal version of our estimates. The second reason is that the function $f(t, x, \xi)$ is not a classical symbol in the phase space $\mathbb{R}_{t} \times \mathbb{R}_{x}^{n} \times \mathbb{R}_{\tau} \times \mathbb{R}_{\xi}^{n}$ and we have to pay attention to the discrepancy between homogeneous localization in the phase space $\mathbb{R}^{2 n+2}$ and localization in $\mathbb{R}^{2 n}$ with parameter $t$. That difficulty should be taken seriously, since the loss of derivatives is strictly larger than 1 ; in fact, commuting a cutoff function with the operator will produce an error of order 0 , larger than what is controlled by the estimate. In the next section, we prove a localized version of the theorem 4.1.9, which will be suitable for future use in the homogeneous framework.

\subsection{From semi-classical to localized inhomogeneous estimates}

We begin with a modified version of Lemma 4.1.7, involving a microlocalization in $\mathbb{R}^{2 n}$.

TOME $134-2006-\mathrm{N}^{\mathrm{O}} 4$ 
LEMMA 4.2.1. - Let $f(t, x, \xi)$ be real-valued satisfying (2.1.2) and (4.1.1); we shall note $F(t)=f(t, x, \xi)^{w}$. Let $\mathcal{M}$ be defined in (4.1.10). We define $c_{1}=$ $c_{0} / C^{2}$, where $c_{0}$ is given by lemma 4.1 .1 and $C$ appears in $(4.1 .26)$. Let $\psi(x, \xi)$ be a real-valued symbol in $S\left(1,\langle\xi\rangle^{-1} \Upsilon\right)$. We have

$$
\begin{aligned}
\frac{d}{d t}\left(\psi^{w} \mathcal{M}(t) \psi^{w}\right)+2 \operatorname{Re}\left(\psi^{w}\right. & \left.\mathcal{M}(t) \psi^{w} F(t)\right) \\
& \geq c_{1} T^{-1} \psi^{w} \mathcal{M}(t) \mathcal{M}(t) \psi^{w}+S\left(\langle\xi\rangle^{-1}, \Upsilon\right)^{w}
\end{aligned}
$$

Proof. — We compute, using (4.1.25) on the fourth line below,

$$
\begin{aligned}
\frac{d}{d t}( & \left.\psi^{w} \mathcal{M}(t) \psi^{w}\right)+2 \operatorname{Re}\left(\psi^{w} \mathcal{M}(t) \psi^{w} F(t)\right) \\
= & \psi^{w} \dot{\mathcal{M}}(t) \psi^{w}+\psi^{w} \mathcal{M}(t) \psi^{w} F(t)+F(t) \psi^{w} \mathcal{M}(t) \psi^{w} \\
= & \psi^{w}(\dot{\mathcal{M}}(t)+2 \operatorname{Re} \mathcal{M}(t) F(t)) \psi^{w}+\psi^{w} \mathcal{M}(t)\left[\psi^{w}, F(t)\right]+\left[F(t), \psi^{w}\right] \mathcal{M}(t) \psi^{w} \\
\geq & c_{1} T^{-1} \psi^{w} \mathcal{M}(t) \mathcal{M}(t) \psi^{w}+c_{2} \psi^{w} S\left(\langle\xi\rangle^{-1}, \Upsilon\right)^{w} \psi^{w}+ \\
& +\psi^{w}\left[\mathcal{M}(t),\left[\psi^{w}, F(t)\right]\right]+\psi^{w}\left[\psi^{w}, F(t)\right] \mathcal{M}(t)-\left[\psi^{w}, F(t)\right] \mathcal{M}(t) \psi^{w} \\
= & c_{1} T^{-1} \psi^{w} \mathcal{M}(t) \mathcal{M}(t) \psi^{w}+c_{2} \psi^{w} S\left(\langle\xi\rangle^{-1}, \Upsilon\right)^{w} \psi^{w}+ \\
& +\psi^{w}\left[\mathcal{M}(t),\left[\psi^{w}, F(t)\right]\right]+\left[\psi^{w},\left[\psi^{w}, F(t)\right]\right] \mathcal{M}(t)+\left[\psi^{w}, F(t)\right]\left[\psi^{w}, \mathcal{M}(t)\right]
\end{aligned}
$$

Next we analyze each term on the last line. We have

- $\psi^{w}\left[\mathcal{M}(t),\left[\psi^{w}, F(t)\right]\right] \in S\left(d\langle\xi\rangle^{-1 / 2} 1 d^{-1}\langle\xi\rangle^{-1 / 2}, \Upsilon\right)^{w}=S\left(\langle\xi\rangle^{-1}, \Upsilon\right)^{w}$ since

$$
\psi^{w},\left[\psi^{w}, F(t)\right] \in S\left(1,\langle\xi\rangle^{-1} \Upsilon\right)^{w}, \quad \mathcal{M}(t) \in S_{1}\left(d\langle\xi\rangle^{-1 / 2}, d^{-2} \Upsilon\right)^{w},
$$

- $\left[\psi^{w},\left[\psi^{w}, F(t)\right]\right] \mathcal{M}(t) \in S\left(d\langle\xi\rangle^{-3 / 2}, \Upsilon\right)^{w} \subset S\left(\langle\xi\rangle^{-1}, \Upsilon\right)^{w}$ since $d \leq$ $2\langle\xi\rangle^{1 / 2}$ and

$$
\left[\psi^{w},\left[\psi^{w}, F(t)\right]\right] \in S\left(\langle\xi\rangle^{-1},\langle\xi\rangle^{-1} \Upsilon\right)^{w}, \quad \mathcal{M}(t) \in S_{1}\left(d\langle\xi\rangle^{-1 / 2}, d^{-2} \Upsilon\right)^{w},
$$

- $\left[\psi^{w}, F(t)\right]\left[\psi^{w}, \mathcal{M}(t)\right], \in S\left(d\langle\xi\rangle^{-1 / 2}\langle\xi\rangle^{-1 / 2} d^{-1}, \Upsilon\right)^{w}=S\left(\langle\xi\rangle^{-1}, \Upsilon\right)^{w}$ since

$$
\left[\psi^{w}, F(t)\right] \in S\left(1,\langle\xi\rangle^{-1} \Upsilon\right)^{w}, \quad \mathcal{M}(t) \in S_{1}\left(d\langle\xi\rangle^{-1 / 2}, d^{-2} \Upsilon\right)^{w} .
$$

We have proven in particular that

$$
\begin{aligned}
\frac{d}{d t}\left(\psi^{w} \mathcal{M}(t) \psi^{w}\right)+ & 2 \operatorname{Re}\left(\psi^{w} \mathcal{M}(t) \psi^{w} F(t)\right) \\
& =\psi^{w}(\dot{\mathcal{M}}(t)+2 \operatorname{Re} \mathcal{M}(t) F(t)) \psi^{w}+S\left(\langle\xi\rangle^{-1}, \Upsilon\right)^{w}
\end{aligned}
$$

BULLETIN DE LA SOCIÉTÉ MATHÉMATIQUE DE FRANCE 
Also, we have $\frac{d}{d t}\left(\psi^{w} \mathcal{M}(t) \psi^{w}\right)+2 \operatorname{Re}\left(\psi^{w} \mathcal{M}(t) \psi^{w} F(t)\right) \geq c_{1} T^{-1} \psi^{w} \mathcal{M}(t) \mathcal{M}(t) \psi^{w}$ $+S\left(\langle\xi\rangle^{-1}, \Upsilon\right)^{w}$, which is $(4.2 .1)$.

THEOREM 4.2.2. - Let $f(t, x, \xi)$ be a smooth real-valued function defined on $\mathbb{R} \times \mathbb{R}^{n} \times \mathbb{R}^{n}$, satisfiying (2.1.2) and (4.1.1). Let $f_{0}(t, x, \xi)$ be a smooth complexvalued function defined on $\mathbb{R} \times \mathbb{R}^{n} \times \mathbb{R}^{n}$, such that $\langle\xi\rangle f_{0}(t, x, \xi)$ satisfies (4.1.1). We define

$$
L=D_{t}+i f(t, x, \xi)^{w}+f_{0}(t, x, \xi)^{w} .
$$

Let $\psi(x, \xi) \in S\left(1,\langle\xi\rangle^{-1} \Upsilon\right)$ be a real-valued symbol. Then there exists $T_{0}>$ $0, c_{0}>0, C \geq 0$, depending on a finite number of seminorms of $f, f_{0}, \psi$, such that, for all $T \leq T_{0}$, all $u \in C_{c}^{\infty}\left((-T, T) ; \mathcal{S}\left(\mathbb{R}^{n}\right)\right)$, with $\omega_{0}$ given by (4.1.29),

$$
\begin{aligned}
& T\left\|\psi^{w}\left(e^{-\omega_{0}}\right)^{w} L u\right\|_{L^{2}\left(\mathbb{R}^{n+1}\right)} \\
& +C T^{1 / 2}\left(\int\|u(t)\|_{H^{-1 / 2}\left(\mathbb{R}^{n}\right)}^{2} d t\right)^{1 / 2} \\
& \quad+C\left(\int\|u(t)\|_{H^{-3 / 2}\left(\mathbb{R}^{n}\right)}^{2} d t\right)^{1 / 2} \geq c_{0}\left(\int\left\|\psi^{w} u(t)\right\|_{H^{-1 / 2}\left(\mathbb{R}^{n}\right)}^{2} d t\right)^{1 / 2} .
\end{aligned}
$$

Proof. - We compute, noting $F(t)=f(t, x, \xi)^{w}$,

$$
\begin{aligned}
& 2 \operatorname{Re}\left\langle L u, i \psi^{w} \mathcal{M}(t) \psi^{w} u\right\rangle=\left\langle\left(\psi^{w} \dot{\mathcal{M}}(t) \psi^{w}+2 \operatorname{Re}\left(\psi^{w} \mathcal{M}(t) \psi^{w} F(t)\right)\right) u, u\right\rangle \\
& +\left\langle\left[\left(\operatorname{Re} f_{0}(t)\right)^{w}, i \psi^{w} \mathcal{M}(t) \psi^{w}\right] u, u\right\rangle+2 \operatorname{Re}\left\langle\psi^{w} \mathcal{M}(t) \psi^{w} \operatorname{Im} f_{0}(t)^{w} u, u\right\rangle .
\end{aligned}
$$

(i) Let us assume that $\operatorname{Im}\left(f_{0}\right) \in S\left(\langle\xi\rangle^{-1},\langle\xi\rangle^{-1} \Upsilon\right)$. Then we get that

$$
\psi^{w} \mathcal{M}(t) \psi^{w} \operatorname{Im} f_{0}(t)^{w} \in S\left(d\langle\xi\rangle^{-1 / 2}\langle\xi\rangle^{-1}, \Upsilon\right)^{w} \subset S\left(\langle\xi\rangle^{-1}, \Upsilon\right)^{w}
$$

and since $\left[\left(\operatorname{Re} f_{0}(t)\right)^{w}, i \psi^{w} \mathcal{M}(t) \psi^{w}\right] \in S\left(d\langle\xi\rangle^{-1 / 2}\langle\xi\rangle^{-1 / 2} d^{-1}, \Upsilon\right)^{w}=S\left(\langle\xi\rangle^{-1}, \Upsilon\right)^{w}$, the inequality (4.1.25), the identity (4.2.2) and lemmas 4.1.8 - 4.2.1 show that

$$
\begin{array}{r}
2 \operatorname{Re}\left\langle L u, i \psi^{w} \mathcal{M}(t) \psi^{w} u\right\rangle=\left\langle\left(\psi^{w} \dot{\mathcal{M}}(t) \psi^{w}+2 \operatorname{Re}\left(\psi^{w} \mathcal{M}(t) \psi^{w} F(t)\right)\right) u, u\right\rangle \\
\geq \frac{c_{1}}{2} T^{-1} \int\left\|\mathcal{M}(t) \psi^{w} u(t)\right\|_{L^{2}\left(\mathbb{R}^{n}\right)}^{2} d t+\frac{c_{0}}{2} T^{-1} \int\left\|\psi^{w} u(t)\right\|_{H^{-1 / 2}\left(\mathbb{R}^{n}\right)}^{2} d t \\
-C \int\|u(t)\|_{H^{-1 / 2}\left(\mathbb{R}^{n}\right)}^{2} d t .
\end{array}
$$

As a consequence, we have

$$
\begin{aligned}
2 T \int\left\|\psi^{w} L u(t)\right\|_{L^{2}\left(\mathbb{R}^{n}\right)}\left\|\mathcal{M}(t) \psi^{w} u(t)\right\|_{L^{2}\left(\mathbb{R}^{n}\right)} d t+C T \int\|u(t)\|_{H^{-1 / 2}\left(\mathbb{R}^{n}\right)}^{2} d t \\
\geq \frac{c_{1}}{2} \int\left\|\mathcal{M}(t) \psi^{w} u(t)\right\|_{L^{2}\left(\mathbb{R}^{n}\right)}^{2} d t+\frac{c_{0}}{2} \int\left\|\psi^{w} u(t)\right\|_{H^{-1 / 2}\left(\mathbb{R}^{n}\right)}^{2} d t,
\end{aligned}
$$

TOME $134-2006-\mathrm{N}^{\mathrm{O}} 4$ 
so that, with $\alpha>0$,

$$
\begin{gathered}
T \int\left(T \alpha^{-1}\left\|\psi^{w} L u(t)\right\|_{L^{2}\left(\mathbb{R}^{n}\right)}^{2}+\alpha T^{-1}\left\|\mathcal{M}(t) \psi^{w} u(t)\right\|_{L^{2}\left(\mathbb{R}^{n}\right)}^{2}\right) d t \\
+C T \int\|u(t)\|_{H^{-1 / 2}\left(\mathbb{R}^{n}\right)}^{2} d t \\
\quad+C T \\
\geq \frac{c_{1}}{2} \int\left\|\mathcal{M}(t) \psi^{w} u(t)\right\|_{L^{2}\left(\mathbb{R}^{n}\right)}^{2} d t+\frac{c_{0}}{2} \int\left\|\psi^{w} u(t)\right\|_{H^{-1 / 2}\left(\mathbb{R}^{n}\right)}^{2} d t .
\end{gathered}
$$

Choosing $\alpha \leq c_{1} / 2$ yields the result

$$
\begin{aligned}
T^{2} \alpha^{-1} \int\left\|\psi^{w} L u(t)\right\|_{L^{2}\left(\mathbb{R}^{n}\right)}^{2} d t+C T \int\|u(t)\|_{H^{-1 / 2}\left(\mathbb{R}^{n}\right)}^{2} d t & \\
& \geq \frac{c_{0}}{2} \int\left\|\psi^{w} u(t)\right\|_{H^{-1 / 2}\left(\mathbb{R}^{n}\right)}^{2} d t,
\end{aligned}
$$

which is a better estimate than the sought one.

(ii) Let us deal now with the general case $\operatorname{Im}\left(f_{0}\right) \in S\left(1,\langle\xi\rangle^{-1} \Upsilon\right)$. Using the definitions (4.1.29), (4.1.31) and the property (4.1.30), we can use (i) above to get the estimate for $L_{0}$, so that with a fixed $c_{2}>0$

$$
\begin{gathered}
T\left\|\psi^{w} L_{0} u\right\|_{L^{2}\left(\mathbb{R}^{n+1}\right)}+T^{1 / 2}\left(\int\|u(t)\|_{H^{-1 / 2}\left(\mathbb{R}^{n}\right)}^{2} d t\right)^{1 / 2} \\
\quad \geq c_{2}\left(\int\left\|\psi^{w} u(t)\right\|_{H^{-1 / 2}\left(\mathbb{R}^{n}\right)}^{2} d t\right)^{1 / 2}
\end{gathered}
$$

so that

$$
\begin{aligned}
T\left\|\psi^{w}\left(e^{-\omega_{0}}\right)^{w} L\left(e^{\omega_{0}}\right)^{w} u\right\|_{L^{2}\left(\mathbb{R}^{n+1}\right)} & +T^{1 / 2}\left(\int\|u(t)\|_{H^{-1 / 2}\left(\mathbb{R}^{n}\right)}^{2} d t\right)^{1 / 2} \\
& \geq c_{2}\left(\int\left\|\psi^{w} u(t)\right\|_{H^{-1 / 2}\left(\mathbb{R}^{n}\right)}^{2} d t\right)^{1 / 2} .
\end{aligned}
$$

Applying this to $u(t)$ given by (4.1.33), we obtain

$$
\begin{aligned}
T\left\|\psi^{w}\left(e^{-\omega_{0}}\right)^{w} L v\right\|_{L^{2}\left(\mathbb{R}^{n+1}\right)}+T^{1 / 2} & \left(\int\left\|\left(\left(e^{\omega_{0}}\right)^{w}\right)^{-1} v(t)\right\|_{H^{-1 / 2}\left(\mathbb{R}^{n}\right)}^{2} d t\right)^{1 / 2} \\
& \geq c_{2}\left(\int\left\|\psi^{w}\left(\left(e^{\omega_{0}}\right)^{w}\right)^{-1} v(t)\right\|_{H^{-1 / 2}\left(\mathbb{R}^{n}\right)}^{2} d t\right)^{1 / 2} .
\end{aligned}
$$

Using that $\left(\left(e^{\omega_{0}}\right)^{w}\right)^{-1}$ is a pseudodifferential operator with symbol in $S\left(1,\langle\xi\rangle^{-1} \Upsilon\right)$, we obtain, using the notation (4.1.35),

$$
\begin{aligned}
& \text { (4.2.7) } T\left\|\psi^{w}\left(e^{-\omega_{0}}\right)^{w} L v\right\|_{L^{2}\left(\mathbb{R}^{n+1}\right)}+C T^{1 / 2}\left(\int\|v(t)\|_{H^{-1 / 2}\left(\mathbb{R}^{n}\right)}^{2} d t\right)^{1 / 2} \\
& \geq c_{2}\left(\int\left\|\Omega(t)^{-1}\left(\langle\xi\rangle^{-1 / 2}\right)^{w} \psi^{w} v(t)\right\|_{L^{2}\left(\mathbb{R}^{n}\right)}^{2} d t\right)^{1 / 2}-C_{1}\left(\int\|v(t)\|_{H^{-3 / 2}\left(\mathbb{R}^{n}\right)}^{2} d t\right)^{1 / 2},
\end{aligned}
$$

BULLETIN DE LA SOCIÉTÉ MATHÉMATIQUe DE FRANCE 
so that, using (4.1.36),

$$
\begin{aligned}
& T\left\|\psi^{w}\left(e^{-\omega_{0}}\right)^{w} L v\right\|_{L^{2}\left(\mathbb{R}^{n+1}\right)}+C T^{1 / 2}\left(\int\|v(t)\|_{H^{-1 / 2}\left(\mathbb{R}^{n}\right)}^{2} d t\right)^{1 / 2} \\
& +C_{1}\left(\int\|v(t)\|_{H^{-3 / 2}\left(\mathbb{R}^{n}\right)}^{2} d t\right)^{1 / 2} \\
& \geq c_{2}\left(\int\left\|\left(\langle\xi\rangle^{-1 / 2}\right)^{w} \psi^{w} v(t)\right\|_{L^{2}\left(\mathbb{R}^{n}\right)}^{2} \frac{1}{\|\Omega(t)\|^{2}} d t\right)^{1 / 2} \\
& \geq c_{3}\left(\int\left\|\left(\langle\xi\rangle^{-1 / 2}\right)^{w} \psi^{w} v(t)\right\|_{L^{2}\left(\mathbb{R}^{n}\right)}^{2} d t\right)^{1 / 2} \\
& =c_{3}\left(\int\left\|\psi^{w} v(t)\right\|_{H^{-1 / 2}\left(\mathbb{R}^{n}\right)}^{2} d t\right)^{1 / 2},
\end{aligned}
$$

which is the result. The proof of the theorem is complete.

\subsection{From inhomogeneous localization to homogeneous localization}

In this section, we are given a positive integer $n$, and we define $N=n+1$. The running point of $T^{*}\left(\mathbb{R}^{N}\right)$ will be denoted by $(y, \eta)$. We are also given a point $\left(y_{0} ; \eta_{0}\right) \in \mathbb{R}^{N} \times \mathbb{S}^{N-1}$ such that

$Y_{0}=\left(y_{0} ; \eta_{0}\right)=\left(t_{0}, x_{0} ; \tau_{0}, \xi_{0}\right) \in \mathbb{R} \times \mathbb{R}^{n} \times \mathbb{R} \times \mathbb{R}^{n}$, with $\tau_{0}=0, \xi_{0} \in \mathbb{S}^{n-1}, t_{0}=0$.

We consider $F(t, x, \xi)=f(t, x, \xi)-i f_{0}(t, x, \xi)$, with $f, f_{0}$ satisfying the assumptions of Theorem 4.2.2. Let $\psi_{0}(\xi)$ be a function supported in a conic neighborhood of $\xi_{0}$ and $\chi_{0}(\tau, \xi)$ be an homogeneous localization near $\tau=0$ as in the appendix A.7 with some positive $r_{0}$. We consider also a classical firstorder pseudodifferential operator $R$ in $\mathbb{R}^{N}$ such that $Y_{0} \notin W F R$. We consider the first-order operator

$$
\mathcal{L}=D_{t}+i\left(F(t, x, \xi) \psi_{0}(\xi) \chi_{0}(\tau, \xi)\right)^{w}+R .
$$

We have

(4.3.3) $\mathcal{L}=D_{t}+i\left(F(t, x, \xi) \psi_{0}(\xi)\right)^{w}+\underbrace{i\left(F(t, x, \xi) \psi_{0}(\xi)\left(\chi_{0}(\tau, \xi)-1\right)\right)^{w}}_{=F_{1}(t, x, \tau, \xi)^{w}}+R$.

Let $\psi_{1}(\xi)$ be a function supported in a conic neighborhood of $\xi_{0}$ and $\chi_{1}(\tau, \xi)$ be an homogeneous localization near $\tau=0$ as in the appendix A.7 with some positive $r_{1}<r_{0}$ and such that

$$
\begin{gathered}
\operatorname{supp} \chi_{1} \subset\left\{\chi_{0}=1\right\}, \quad \operatorname{supp}\left(\psi_{1} \chi_{1}\right) \subset\left\{\psi_{0} \chi_{0}=1\right\}, \\
{\left[-T_{1}, T_{1}\right] \times K_{1} \times \operatorname{supp} \psi_{1} \chi_{1} \subset(W F R)^{c},}
\end{gathered}
$$

TOME $134-2006-\mathrm{N}^{\mathrm{O}} 4$ 
where $T_{1}>0$ and $K_{1}$ is a compact neighborhood of $x_{0}$. Let $\psi(x, \xi)$ be a symbol satisfying the assumptions of Theorem 4.2 .2 and let $\rho_{1} \in C_{\mathrm{c}}^{\infty}(\mathbb{R})$, such that

$$
\operatorname{supp} \psi \subset K_{1} \times\left\{\psi_{1}=1\right\}, \quad \operatorname{supp} \rho_{1} \subset\left[-T_{1}, T_{1}\right] .
$$

We can apply the theorem 4.2 .2 to the operator $L=D_{t}+i\left(F(t, x, \xi) \psi_{0}(\xi)\right)^{w}$. We have, with $u \in \mathcal{S}\left(\mathbb{R}^{N}\right)$,

$$
\begin{gathered}
T_{1}\left\|\psi^{w}\left(e^{-\omega_{0}}\right)^{w}\left(\mathcal{L}-F_{1}-R\right) \rho_{1} \chi_{1}^{w} u\right\|_{L^{2}\left(\mathbb{R}^{n+1}\right)} \\
+C T_{1}^{1 / 2}\left(\int\left\|\rho_{1} \chi_{1}^{w} u(t)\right\|_{H^{-1 / 2}\left(\mathbb{R}^{n}\right)}^{2} d t\right)^{1 / 2}+C\left(\int\left\|\rho_{1} \chi_{1}^{w} u(t)\right\|_{H^{-3 / 2}\left(\mathbb{R}^{n}\right)}^{2} d t\right)^{1 / 2} \\
\geq c_{0}\left(\int\left\|\psi^{w} \rho_{1} \chi_{1}^{w} u(t)\right\|_{H^{-1 / 2}\left(\mathbb{R}^{n}\right)}^{2} d t\right)^{1 / 2} .
\end{gathered}
$$

We get then

$$
\begin{aligned}
& \quad T_{1}\left\|\psi^{w}\left(e^{-\omega_{0}}\right)^{w} \rho_{1} \chi_{1}^{w} \mathcal{L} u+\psi^{w}\left(e^{-\omega_{0}}\right)^{w} \rho_{1}\left[\mathcal{L}, \chi_{1}^{w}\right] u\right\|_{L^{2}\left(\mathbb{R}^{n+1}\right)} \\
& +T_{1}\left\|\psi^{w}\left(e^{-\omega_{0}}\right)^{w}\left[\mathcal{L}, \rho_{1}\right] \chi_{1}^{w} u\right\|_{L^{2}\left(\mathbb{R}^{n+1}\right)} \\
& +T_{1}\left\|\psi^{w}\left(e^{-\omega_{0}}\right)^{w} F_{1}^{w} \rho_{1} \chi_{1}^{w} u\right\|_{L^{2}\left(\mathbb{R}^{n+1}\right)}+T_{1}\left\|\psi^{w}\left(e^{-\omega_{0}}\right)^{w} R \rho_{1} \chi_{1}^{w} u\right\|_{L^{2}\left(\mathbb{R}^{n+1}\right)} \\
& +C T_{1}^{1 / 2}\left\|\left(\langle\xi\rangle^{-1 / 2}\right)^{w} \rho_{1} \chi_{1}^{w} u\right\|_{L^{2}\left(\mathbb{R}^{n+1}\right)}+C\left\|\left(\langle\xi\rangle^{-3 / 2}\right)^{w} \rho_{1} \chi_{1}^{w} u\right\|_{L^{2}\left(\mathbb{R}^{n+1}\right)} \\
& \quad \geq c_{0}\left(\int\left\|\psi^{w} \rho_{1} \chi_{1}^{w} u\right\|_{H^{-1 / 2}\left(\mathbb{R}^{n}\right)}^{2} d t\right)^{1 / 2} .
\end{aligned}
$$

We assume now that $u \in \mathcal{S}\left(\mathbb{R}^{N}\right)$, supp $u \subset\left\{(t, x),|t| \leq T_{1} / 2\right\}$ and also that $\rho_{1}$ is 1 on $\left[-3 T_{1} / 4,3 T_{1} / 4\right]$. We introduce two admissible ${ }^{(5)}$ metrics on $\mathbb{R}^{2 N}$,

$G=|d t|^{2}+|d x|^{2}+\frac{|d \xi|^{2}+|d \tau|^{2}}{1+|\xi|^{2}+\tau^{2}} \leq g=|d t|^{2}+|d x|^{2}+\frac{|d \xi|^{2}}{1+|\xi|^{2}}+\frac{|d \tau|^{2}}{1+|\xi|^{2}+\tau^{2}}$.

(1) The operator $\left[\mathcal{L}, \chi_{1}^{w}\right]$ has a symbol in $S(1, G)$ which is essentially supported in the region where $|\tau| \sim|\xi|$.

(2) The quantity $\left[\mathcal{L}, \rho_{1}\right] \chi_{1}^{w} u=\left[\mathcal{L}, \rho_{1}\right] \chi_{1}^{w} \rho_{2} u$ if $\rho_{2}(t)$ is 1 on $\left[-T_{1} / 2, T_{1} / 2\right]$ and supported in $\left[-3 T_{1} / 4,3 T_{1} / 4\right]$ and thus the operator $\left[\mathcal{L}, \rho_{1}\right] \chi_{1}^{w} \rho_{2}$ has a symbol in $S\left((1+|\xi|+|\tau|)^{-\infty}, G\right)$.

(3) The operator $F_{1}^{w} \rho_{1} \chi_{1}^{w}$ is the composition of the symbol $F_{1} \in S(\langle\xi\rangle, g)$ with the symbol in $\rho_{1} \sharp \chi_{1} \in S(1, G)$ and thus is a priori in $S(\langle\xi\rangle, g)$; however, looking at the expansion, and using (4.3.4), we see that it has a symbol in $S\left((1+|\xi|+|\tau|)^{-\infty}, G\right)$ : it is not completely obvious though and we refer the reader to the lemma A.8.1 for a complete argument.

(5) The properties of definition 1.3 .1 are classical for $G$ and easily checked for $g$. One can check also that $(1+|\xi|+|\tau|)^{s}$ are $G$-weights and $(1+|\xi|)^{s}$ are $g$-weights.

BULletin DE LA SOCiÉtÉ MATHÉmATiQUe DE FRANCE 
(4) The operator $\psi^{w}\left(e^{-\omega_{0}}\right)^{w} R \rho_{1} \chi_{1}^{w}$ is also the composition of an operator in $S(1, g)^{w}$ with an operator in $S(\langle\xi, \tau\rangle, G)^{w}$; however, using (4.3.4-5-6) and the appendix A.8, we see that $\psi^{w}\left(e^{-\omega_{0}}\right)^{w} R \rho_{1} \chi_{1}^{w}$ has a symbol in $S\left((1+|\xi|+|\tau|)^{-\infty}, G\right)$.

(5) The operator $\left(\langle\xi\rangle^{s}\right)^{w} \rho_{1} \chi_{1}^{w}$ is also the sum of an operator in $S\left(\langle\tau, \xi\rangle^{s}, G\right)$ plus a symbol in $S\left((1+|\xi|+|\tau|)^{-\infty}, G\right)$.

We write now, with $R_{1}$ of order $-\infty$ (weight $\langle\xi, \tau\rangle^{-\infty}$ ) for $G, E_{0}$ of order 0 (weight 1) for $G$, supported in $\left\{(t, x, \tau, \xi),|t| \leq T_{1}, x \in K_{1},(\tau, \xi) \in\right.$ $\left.\operatorname{supp} \nabla \chi_{1},(x, \xi) \in \operatorname{supp} \psi\right\}$,

$$
\begin{gathered}
T_{1}\left\|\psi^{w}\left(e^{-\omega_{0}}\right)^{w} \rho_{1} \chi_{1}^{w} \mathcal{L} u+E_{0} u\right\|_{L^{2}\left(\mathbb{R}^{n+1}\right)}+T_{1}\left\|R_{1} u\right\|_{L^{2}\left(\mathbb{R}^{n+1}\right)} \\
+C T_{1}^{1 / 2}\|u\|_{H^{-1 / 2}\left(\mathbb{R}^{n+1}\right)}+C\|u\|_{H^{-3 / 2}\left(\mathbb{R}^{n+1}\right)} \\
\geq c_{0}\left(\int\left\|\psi^{w} \rho_{1} \chi_{1}^{w} u\right\|_{H^{-1 / 2}\left(\mathbb{R}^{n}\right)}^{2} d t\right)^{1 / 2} \cdot
\end{gathered}
$$

THEOREM 4.3.1. - Let $\mathcal{L}$ be the pseudodifferential operator given by (4.3.2) and $Y_{0}=\left(y_{0}, \eta_{0}\right)$ be given by (4.3.1). We assume that $\left\{Y_{0}\right\} \subset \Delta_{0} \subset(W F R)^{c}$, where $\Delta_{0}$ is a compact-conic neighborhood of $Y_{0}$. Then, there exists two pseudodifferential operators $\Phi_{0}, \Psi_{0}$ of order 0 (weight 1) for $G$, both essentially supported in $\Delta_{0}$ with $\Phi_{0}$ is elliptic at $Y_{0}$, and there exists $r>0$ such that, for all $u \in \mathcal{S}\left(\mathbb{R}^{N}\right), \operatorname{supp} u \subset\{(t, x),|t| \leq r\}$,

$$
r\left\|\Psi_{0} \mathcal{L} u\right\|_{L^{2}\left(\mathbb{R}^{N}\right)}+r^{1 / 2}\|u\|_{H^{-1 / 2}\left(\mathbb{R}^{N}\right)}+\|u\|_{H^{-3 / 2}\left(\mathbb{R}^{N}\right)} \geq\left\|\Phi_{0} u\right\|_{H^{-1 / 2}\left(\mathbb{R}^{N}\right)} .
$$

Proof. - It is a direct consequence of (4.3.9) since, using the ellipticity of $\mathcal{L}$ in the support of the symbol of $E_{0}$, we get $E_{0}=\mathcal{K} \mathcal{L}+R_{2}$, where $\mathcal{K}$ is a pseudodifferential operator of order 0 such that $W F \mathcal{K} \subset \Delta_{0}$ and $R_{2}$ is a pseudodifferential operator of order $-\infty$ for $G$.

4.4. Proof of the solvability result stated in Theorem 1.2.2. - Let $P$ be a first-order pseudodifferential operator with principal symbol $p$ satisfying the assumptions of Theorem 1.2.2 and let $\left(y_{0}, \eta_{0}\right)$ be a point in the cosphere bundle. If $p\left(y_{0}, \eta_{0}\right) \neq 0$, then there exists a pseudodifferential operator $\Phi_{0}$ of order 0 , elliptic at $\left(y_{0}, \eta_{0}\right)$ such that

$$
\left\|P^{*} u\right\|_{0}+\|u\|_{-1} \geq\left\|\Phi_{0} u\right\|_{1} .
$$

In fact, the ellipticity assumption implies that there exist a pseudodifferential operator $K$ of order -1 and a pseudodifferential operator $R$ of order 0 such that

$$
\mathrm{Id}=K P^{*}+R, \quad\left(y_{0}, \eta_{0}\right) \notin W F R .
$$

As consequence, for $\Phi_{0}$ of order 0 essentially supported close enough to $\left(y_{0}, \eta_{0}\right)$, we get $\Phi_{0}=\Phi_{0} K P^{*}+\Phi_{0} R$ with $\Phi_{0} R$ of order $-\infty$, which gives (4.4.1).

TOME $134-2006-\mathrm{N}^{\mathrm{O}} 4$ 
Let us assume now that $p\left(y_{0}, \eta_{0}\right)=0$. We know from the assumption (1.2.1) that $\partial_{\eta} p\left(y_{0}, \eta_{0}\right) \neq 0$ and we may suppose that $\left(\partial_{\eta} \operatorname{Re} p\right)\left(y_{0}, \eta_{0}\right) \neq 0$. Using the Malgrange-Weierstrass theorem, we can find a conic neighborhood of $\left(y_{0}, \eta_{0}\right)$ in which

$$
p(y, \eta)=(\sigma+a(s, z, \zeta)+i b(s, z, \zeta)) e_{0}(y, \eta)
$$

where $a, b$ are real-valued positively homogeneous of degree $1, e_{0}$ is homogeneous of degree 0 , elliptic near $\left(y_{0}, \eta_{0}\right),(s, z ; \sigma, \zeta) \in \mathbb{R} \times \mathbb{R}^{n} \times \mathbb{R} \times \mathbb{R}^{n}$ a choice of symplectic coordinates in $T^{*}\left(\mathbb{R}^{N}\right)(N=n+1)$, with $y_{0}=(0,0), \eta_{0}=$ $(0, \ldots, 0,1)$. Noting that the Poisson bracket

$$
\{\sigma+a, s\}=1
$$

we see that there exists an homogeneous canonical transformation $\Xi^{-1}$, from a (conic) neighborhood of $\left(y_{0}, \eta_{0}\right)$ to a conic neighborhood of $(0 ; 0, \ldots 0,1)$ in $\mathbb{R}^{N} \times \mathbb{R}^{N}$ such that

$$
p \circ \Xi=(\tau+i q(t, x, \xi))(e \circ \Xi) .
$$

Note in particular that, setting $\tau=\sigma+a, t=s$, (which preserves the coordinate s) yields

$$
-\partial_{\tau} q=\{t, q\}=\{s, b\} \circ \chi=0 .
$$

We see now that there exists some elliptic Fourier integral operators $A, B$ and $E$ a pseudodifferential operator of order 0 , elliptic at $\left(y_{0}, \eta_{0}\right)$ such that

$$
\begin{aligned}
A E P^{*} B & =D_{t}+i\left(f(t, x, \xi) \chi_{0}(\tau, \xi)\right)^{w}+R, \\
B A & =\operatorname{Id}+S,\left(y_{0}, \eta_{0}\right) \in \Gamma_{0}\left(\text { conic neighborhood of }\left(y_{0}, \eta_{0}\right)\right) \subset(W F S)^{c},
\end{aligned}
$$

where $f$ satisfies (2.1.2), $R$ is a pseudodifferential operator of order 0 , and $\chi_{0}$ is a nonnegative homogeneous localization near $\tau=0$. Using the fact that the coordinate $s$ is preserved by the canonical transformation, we can assume that $A, B$ are local operators in the $t$ variable, i.e., are such that

$$
\begin{aligned}
u \in C_{\mathrm{c}}^{\infty}, \operatorname{supp} u \subset\left\{(t, x) \in \mathbb{R} \times \mathbb{R}^{n},|t|\right. & \leq r\} \\
& \Longrightarrow \operatorname{supp} B u \subset\left\{(s, z) \in \mathbb{R} \times \mathbb{R}^{n},|s| \leq r\right\} .
\end{aligned}
$$

Using the fact that the operator $P$ is polyhomogeneous, one can iterate the use of the Malgrange-Weierstrass theorem to reduce our case to $A E P^{*} B=\mathcal{L}$ of the type given in (4.3.2). We can apply the theorem 4.3.1, giving the existence of a pseudodifferential operator $\Psi_{0}$ of order 0 , elliptic at $\Xi^{-1}\left(y_{0}, \eta_{0}\right)$, essentially supported in $\Xi^{-1}\left(\Gamma_{0}\right)$ such that for all $u \in C_{\mathrm{c}}^{\infty}\left(\mathbb{R}^{N}\right)$, supp $u \subset\{|t| \leq r\}$,

$$
r\left\|\Psi_{0} A E P^{*} B u\right\|_{0}+r^{1 / 2}\|u\|_{-1 / 2}+\|u\|_{-3 / 2} \geq\left\|\Phi_{0} u\right\|_{-1 / 2} .
$$


We may assume that $A$ and $B$ are properly supported and apply the previous inequality to $u=A v$, whose support in the $s$ variable is unchanged. We get

$$
r\left\|\Psi_{0} A E P^{*} B A v\right\|_{0}+r^{1 / 2}\|A v\|_{-1 / 2}+\|A v\|_{-3 / 2} \geq\left\|\Phi_{0} A v\right\|_{-1 / 2},
$$

so that

$$
\begin{aligned}
r\left\|\Psi_{0} A E P^{*} v\right\|_{0}+C r^{1 / 2}\|v\|_{-1 / 2}+C_{1}\|v\|_{-3 / 2} & \\
& \geq\left\|\Phi_{0} A v\right\|_{-1 / 2} \geq C_{2}^{-1}\left\|B \Phi_{0} A v\right\|_{-1 / 2},
\end{aligned}
$$

which gives, for all $v \in C_{\mathrm{c}}^{\infty}\left(\mathbb{R}^{N}\right), \operatorname{supp} v \subset\left\{y \in \mathbb{R}^{N},\left|y-y_{0}\right| \leq r\right\}$,

$$
r\left\|P^{*} v\right\|_{0}+r^{1 / 2}\|v\|_{-1 / 2}+\|v\|_{-3 / 2} \geq\|\Phi v\|_{-1 / 2},
$$

where $\Phi=c B \Phi_{0} A$ is a pseudodifferential operator of order 0 , elliptic near $\left(y_{0}, \eta_{0}\right)$. By compactness of the cosphere bundle, one gets, using (4.4.2) or (4.4.1),

$$
\begin{aligned}
\|v\|_{-1 / 2} \leq C \sum_{1 \leq \kappa \leq l}\left\|\Phi_{0 \kappa} v\right\|_{-1 / 2}+C\|v\|_{-1} & \\
& \leq C_{1} r\left\|P^{*} v\right\|_{0}+C_{1} r^{1 / 2}\|v\|_{-1 / 2}+C_{1}\|v\|_{-1},
\end{aligned}
$$

which entails, by shrinking $r$, the existence of $r_{0}>0, C_{0}>0$, such that for $v \in C_{\mathrm{c}}^{\infty}\left(\mathbb{R}^{N}\right), \operatorname{supp} v \subset\left\{y \in \mathbb{R}^{N},\left|y-y_{0}\right| \leq r_{0}\right\}=B_{r_{0}}$,

$$
\|v\|_{-1 / 2} \leq C_{0}\left\|P^{*} v\right\|_{0} \text {. }
$$

Let $s$ be a real number and $P$ be an operator of order $m$, satisfying the assumptions of Theorem 1.2.2. Let $E_{\sigma}$ be a properly supported operator with symbol $\langle\xi\rangle^{\sigma}$. Then the operator $E_{1-m-s} P E_{s}$ is of first order, satisfies condition $(\psi)$ and from the previous discussion, there exists $C_{0}>0, r_{0}>0$ such that

$$
\|v\|_{-1 / 2} \leq C_{0}\left\|E_{s} P^{*} E_{1-m-s} v\right\|_{0}, \quad v \in C_{\mathrm{c}}^{\infty}\left(\mathbb{R}^{N}\right), \operatorname{supp} v \subset B_{r_{0}} .
$$

We get, with $\chi_{r}$ supported in $B_{r}$ and $\chi_{r}=1$ on $B_{r / 2}$, with $\operatorname{supp} u \subset B_{r_{0} / 4}$,

$$
\begin{gathered}
\left\|\chi_{r_{0}} E_{m+s-1} \chi_{r_{0} / 2} u\right\|_{-1 / 2} \leq C_{0}\left\|E_{s} P^{*} E_{1-m-s} \chi_{r_{0}} E_{m+s-1} \chi_{r_{0} / 2} u\right\|_{0} \\
\leq C_{0}\|E_{s} P^{*} E_{1-m-s} \underbrace{\left[\chi_{r_{0}}, E_{m+s-1}\right] \chi_{r_{0} / 2}}_{S^{-\infty}} u\|_{0} \\
+C_{0}\|E_{s} P^{*} \underbrace{E_{1-m-s} E_{m+s-1}}_{=\mathrm{Id}+S^{-\infty}} \underbrace{\chi_{r_{0} / 2} u}_{=u}\|_{0} \\
\leq C_{0}\left\|P^{*} u\right\|_{s}+\|R u\|_{0},
\end{gathered}
$$

where $R$ is of order $-\infty$. Since we have

$$
\chi_{r_{0}} E_{m+s-1} \chi_{r_{0} / 2} u=\underbrace{\left[\chi_{r_{0}}, E_{m+s-1}\right] \chi_{r_{0} / 2}}_{S^{-\infty}} u+E_{m+s-1} \underbrace{\chi_{r_{0}} \chi_{r_{0} / 2} u}_{=u},
$$

TOME $134-2006-\mathrm{N}^{\mathrm{O}} 4$ 
we get $\|u\|_{s+m-\frac{3}{2}} \leq C_{0}\left\|P^{*} u\right\|_{s}+C_{1}\|u\|_{s+m-2}$ and, shrinking the support of $u$, we obtain the estimate

$$
\|u\|_{s+m-\frac{3}{2}} \leq C_{2}\left\|P^{*} u\right\|_{s}
$$

for $u \in C_{\mathrm{c}}^{\infty}$ with support in a neighborhood of $y_{0}$. This implies the local solvability of $P$, with the loss of derivatives claimed by the theorem 1.2.2, whose proof is now complete.

\section{Appendix A}

A.1. Wick quantization. - We recall here some facts on the so-called Wick quantization, as used in [21, 22, 23].

Definition A.1.1. - Let $Y=(y, \eta)$ be a point in $\mathbb{R}^{2 n}$. The operator $\Sigma_{Y}$ is defined as $\left[2^{n} e^{-2 \pi|\cdot-Y|^{2}}\right]^{w}$. This is a rank-one orthogonal projection: $\Sigma_{Y} u=$ $(W u)(Y) \tau_{Y} \varphi$ with $(W u)(Y)=\left\langle u, \tau_{Y} \varphi\right\rangle_{L^{2}\left(\mathbb{R}^{n}\right)}$, where $\varphi(x)=2^{n / 4} e^{-\pi|x|^{2}}$ and $\left(\tau_{y, \eta} \varphi\right)(x)=\varphi(x-y) e^{2 i \pi\left\langle x-\frac{y}{2}, \eta\right\rangle}$. Let $a$ be in $L^{\infty}\left(\mathbb{R}^{2 n}\right)$. The Wick quantization of $a$ is defined as

$$
a^{\mathrm{Wick}}=\int_{\mathbb{R}^{2 n}} a(Y) \Sigma_{Y} d Y
$$

The following proposition is classical and easy (see e.g. section 5 in [22]).

\section{Proposition A.1.2}

1. Let $a$ be in $L^{\infty}\left(\mathbb{R}^{2 n}\right)$. Then $a^{\text {Wick }}=W^{*} a^{\mu} W$ and $1^{\text {Wick }}=I d_{L^{2}\left(\mathbb{R}^{n}\right)}$ where $W$ is the isometric mapping from $L^{2}\left(\mathbb{R}^{n}\right)$ to $L^{2}\left(\mathbb{R}^{2 n}\right)$ given above, and $a^{\mu}$ the operator of multiplication by a in $L^{2}\left(\mathbb{R}^{2 n}\right)$. The operator $\pi_{H}=W W^{*}$ is the orthogonal projection on a closed proper subspace $H$ of $L^{2}\left(\mathbb{R}^{2 n}\right)$. Moreover, we have

$$
\begin{gathered}
\left\|a^{\text {Wick }}\right\|_{\mathcal{L}\left(L^{2}\left(\mathbb{R}^{n}\right)\right)} \leq\|a\|_{L^{\infty}\left(\mathbb{R}^{2 n}\right)}, \\
a(X) \geq 0 \text { for all } X \text { implies } a^{\text {Wick }} \geq 0 .
\end{gathered}
$$

2. Let $m$ be a real number, and $p \in S\left(\Lambda^{m}, \Lambda^{-1} \Gamma\right)$. Then $p^{\text {Wick }}=p^{w}+r(p)^{w}$, with $r(p) \in S\left(\Lambda^{m-1}, \Lambda^{-1} \Gamma\right)$ so that the mapping $p \mapsto r(p)$ is continuous. More precisely, one has

$$
r(p)(X)=\int_{0}^{1} \int_{\mathbb{R}^{2 n}}(1-\theta) p^{\prime \prime}(X+\theta Y) Y^{2} e^{-2 \pi \Gamma(Y)} 2^{n} d Y d \theta .
$$

Note that $r(p)=0$ if $p$ is affine.

3. For $a \in L^{\infty}\left(\mathbb{R}^{2 n}\right)$, the Weyl symbol of $a^{\text {Wick }}$ is

$a * 2^{n} \exp -2 \pi \Gamma \quad$ which belongs to $S(1, \Gamma)$ with $k^{\text {th }}$-seminorm $c(k)\|a\|_{L^{\infty}}$.

BULLETIN DE LA SOCiÉTÉ MATHÉMATIQUe DE FRANCE 
4. Let $\mathbb{R} \ni t \mapsto a(t, X) \in \mathbb{R}$ such that, for $t \leq s, a(t, X) \leq a(s, X)$. Then, for $u \in C_{c}^{1}\left(\mathbb{R}_{t}, L^{2}\left(\mathbb{R}^{n}\right)\right)$, assuming $a(t, \cdot) \in L^{\infty}\left(\mathbb{R}^{2 n}\right)$,

$$
\int_{\mathbb{R}} \operatorname{Re}\left\langle D_{t} u(t), i a(t)^{\mathrm{Wick}} u(t)\right\rangle_{L^{2}\left(\mathbb{R}^{n}\right)} d t \geq 0 .
$$

5. With the operator $\Sigma_{Y}$ given in definition A.1.1, we have the estimate

$$
\left\|\Sigma_{Y} \Sigma_{Z}\right\|_{\mathcal{L}\left(L^{2}\left(\mathbb{R}^{n}\right)\right)} \leq 2^{n} e^{-\frac{\pi}{2} \Gamma(Y-Z)} .
$$

6. More precisely, the Weyl symbol of $\Sigma_{Y} \Sigma_{Z}$ is, as a function of the variable $X \in \mathbb{R}^{2 n}$, setting $\Gamma(T)=|T|^{2}$

$$
e^{-\frac{\pi}{2}|Y-Z|^{2}} e^{-2 i \pi[X-Y, X-Z]} 2^{n} e^{-2 \pi\left|X-\frac{Y+Z}{2}\right|^{2}} .
$$

Since for the Weyl quantization, one has $\left\|a^{w}\right\|_{\mathcal{L}\left(L^{2}\left(\mathbb{R}^{n}\right)\right)} \leq 2^{n}\|a\|_{L^{1}\left(\mathbb{R}^{2 n}\right)}$, we get the result (A.1.7) from (A.1.6). Note that (A.1.5) is simply a way of writing that $\frac{d}{d t}\left(a(t)^{\text {Wick }}\right) \geq 0$, which is a consequence of (A.1.3) and of the non-decreasing assumption made on $t \mapsto a(t, X)$.

Lemma A.1.3. - Let $M$ be a $\Gamma$-weight, i.e., a positive function such that $M(X) M(Y)^{-1} \leq C(1+\Gamma(X-Y))^{N}$ (see definition 1.3.1). Then if a measurable function a defined on $\mathbb{R}^{2 n}$ satisfies for all $X,|a(X)| \leq C_{1} M(X)$, the symbol $a * \exp -2 \pi \Gamma$ belongs to $S(M, \Gamma)$ with semi-norms depending only on $C_{1}$. More generally, for a polynomial $p$ the symbol $A$ defined by

$$
A(X)=\int a(Y) p(X-Y) \exp -2 \pi \Gamma(X-Y) d Y
$$

belongs to to $S(M, \Gamma)$.

Proof. - We check first

$(A .1 .8)\left(a * 2^{n} \exp -2 \pi \Gamma\right)^{(k)}(X)=\int a(Y) P_{k}(X-Y) 2^{n} \exp -2 \pi \Gamma(X-Y) d Y$

with a polynomial $P_{k}$, which gives

$$
\begin{aligned}
& M(X)^{-1}\left|\left(a * 2^{n} \exp -2 \pi \Gamma\right)^{(k)}(X)\right| \\
& \leq C_{1} \int \frac{M(Y)}{M(X)}\left|P_{k}(X-Y)\right| 2^{n} \exp -2 \pi \Gamma(X-Y) d Y \\
& \leq C_{1} \int C(1+\Gamma(X-Y))^{N}\left|P_{k}(X-Y)\right| 2^{n} \exp -2 \pi \Gamma(X-Y) d Y=C_{1} C \gamma(k, N, n) .
\end{aligned}
$$

Let us examine $A^{(k)}$ : it is a sum of terms of type (A.1.8) and thus the above argument works.

TOME $134-2006-\mathrm{N}^{\mathrm{O}} 4$ 
LEMMA A.1.4. - Let $g$ be an admissible metric on $\mathbb{R}^{2 n}$ (see definition 1.3.1) such that, with $\Gamma$ a given symplectic norm, there exists $C_{0}>0, n_{0} \geq 0$ such that

$$
\forall X, Y, T, g_{X}(T) \leq C_{0} \Gamma(T), \quad \frac{g_{X}(T)}{g_{Y}(T)} \leq C_{0}(1+\Gamma(X-Y))^{n_{0}} .
$$

Let $m$ be a weight for $g$ (definition 1.3.1) such that

$$
\frac{m(Y)}{m(Z)} \leq C_{0}(1+\Gamma(Z-Y))^{n_{0}} .
$$

Then, if $A \in O p(S(m, g))$, there exists a semi-norm $\gamma$ of the symbol of $A$ such that

$$
|\langle A v, v\rangle| \leq \gamma\left\langle m^{\text {Wick }} v, v\right\rangle=\gamma \int_{\mathbb{R}^{2 n}} m(Y)\left\|\Sigma_{Y} v\right\|_{L^{2}}^{2} d Y
$$

Proof. - Theorem 6.9 in [2] shows that the space $\mathcal{H}\left(m^{1 / 2}, g\right)$ is equal to $\mathcal{H}\left(m^{1 / 2}, \Gamma\right)$ provided that $m^{1 / 2}$ is regular. In fact we may assume that $m$ is regular since it is anyhow always equivalent to a regular weight. Using definition 7.1 in [2], we check that $g$ is dominated by a strongly temperate metric, namely the constant metric $\Gamma$. Moreover the corollary 6.7 and theorem 7.8 in [2] imply

$$
\begin{aligned}
|\langle A v, v\rangle| \leq\|A v\|_{\mathcal{H}\left(m^{-1 / 2}, g\right)}\|v\|_{\mathcal{H}\left(m^{1 / 2}, g\right)} & \leq \gamma\|v\|_{\mathcal{H}\left(m^{1 / 2}, g\right)}^{2} \\
=\gamma\|v\|_{\mathcal{H}\left(m^{1 / 2}, \Gamma\right)}^{2} & =\gamma \int_{\mathbb{R}^{2 n}} m(Y)\left\|\theta_{Y}^{w} u\right\|_{L^{2}}^{2} d Y,
\end{aligned}
$$

where $\left(\theta_{Y}\right)$ is a partition of unity related to the metric $\Gamma$. We have, using the results of this section, (A.1.10) and (A.1.6), with $\langle T\rangle^{2}=1+\Gamma(T)$, for all $N_{1}, N_{2}$,

$$
\begin{aligned}
& \int m(Y)\left\|\theta_{Y}^{w} u\right\|^{2} d Y \\
& \quad=\iiint_{\mathbb{R}^{2 n}} m(Y)\left\langle\theta_{Y}^{w} \Sigma_{Z_{1}} \Sigma_{Z_{1}} u, \theta_{Y}^{w} \Sigma_{Z_{2}} \Sigma_{Z_{2}} u\right\rangle d Y d Z_{1} d Z_{2} \\
& \leq \iiint m\left(Z_{1}\right)^{1 / 2} m\left(Z_{2}\right)^{1 / 2}\left\|\Sigma_{Z_{1}} u\right\|\left\|\Sigma_{Z_{2}} u\right\|\left\langle Y-Z_{1}\right\rangle^{-N_{1}}\left\langle Z_{2}-Z_{1}\right\rangle^{-N_{2}} d Y d Z_{1} d Z_{2} C_{N_{1}, N_{2}} \\
& \leq \iint m\left(Z_{1}\right)^{1 / 2} m\left(Z_{2}\right)^{1 / 2}\left\|\Sigma_{Z_{1}} u\right\|\left\|\Sigma_{Z_{2}} u\right\|\left\langle Z_{2}-Z_{1}\right\rangle^{-N_{2}} d Z_{1} d Z_{2} C_{N_{1}, N_{2}} \\
& \leq \int m(Z)\left\|\Sigma_{Z} u\right\|^{2} d Z
\end{aligned}
$$

which completes the proof of the lemma. 
LemmA A.1.5. - Let $m_{1}, m_{2}$ be two $\Gamma$-weights (see definition 1.3.1) and $a_{1}, a_{2}$ be two locally Lipschitz continuous functions such that $\left|a_{1}(X)\right| \leq$ $m_{1}(X), \quad\left|a_{2}^{\prime}(X)\right| \leq m_{2}(X)$. Then the operator

$$
a_{1}^{\text {Wick }} a_{2}^{\text {Wick }} \in\left(a_{1} a_{2}\right)^{\text {Wick }}+O p\left(S\left(m_{1} m_{2}, \Gamma\right)\right) .
$$

Proof. — We use the definition A.1.1 and Taylor's formula to write

$$
\begin{array}{r}
a_{1}^{\text {Wick }} a_{2}^{\text {Wick }}=\iint a_{1}(Y)\left(a_{2}(Y)+\int_{0}^{1} a_{2}^{\prime}(Y+\theta(Z-Y)) d \theta(Z-Y)\right) \Sigma_{Y} \Sigma_{Z} d Y d Z \\
=\left(a_{1} a_{2}\right)^{\mathrm{Wick}}+R^{w},
\end{array}
$$

with

$$
\begin{aligned}
R(X)=\iiint_{0}^{1} a_{1}(Y) a_{2}^{\prime}( & +\theta(Z-Y))(Z-Y) e^{-\frac{\pi}{2}|Y-Z|^{2}} \times \\
\times & e^{-2 i \pi[X-Y, X-Z]} 2^{n} e^{-2 \pi\left|X-\frac{Y+Z}{2}\right|^{2}} d Y d Z d \theta .
\end{aligned}
$$

We have, using (5) in definition 1.3.1,

$$
\begin{aligned}
& |R(X)| \leq \iiint_{0}^{1} m_{1}(Y) m_{2}(Y) \frac{m_{2}(Y+\theta(Z-Y))}{m_{2}(Y)}|Z-Y| e^{-\frac{\pi}{2}|Y-Z|^{2}} \\
& 2^{n} e^{-2 \pi\left|X-\frac{Y+Z}{2}\right|^{2}} d Y d Z d \theta \\
& \leq C m_{1}(X) m_{2}(X) \iiint_{0}^{1}\left(1+|Y-X|^{2}\right)^{N}\left(1+|Y-Z|^{2}\right)^{N+1 / 2} e^{-\frac{\pi}{2}|Y-Z|^{2}} \\
& \quad e^{-2 \pi\left|\frac{Y+Z}{2}-X\right|^{2}} d Y d Z d \theta \\
& =C m_{1}(X) m_{2}(X) \iint\left(1+|T / 2+S|^{2}\right)^{N}\left(1+|T|^{2}\right)^{N+1 / 2} e^{-\frac{\pi}{2}|T|^{2}} e^{-2 \pi|S|^{2}} d T d S \\
& \quad=C^{\prime} m_{1}(X) m_{2}(X) .
\end{aligned}
$$

Moreover taking derivatives of $R$ in its defining formula (A.1.13) above leads to the same estimate for $R^{(k)}(X)$. The proof of the lemma is complete.

LEMMA A.1.6. - Let $\left(\chi_{k}\right)$ be a partition of unity and $\left(\psi_{k}\right)$ be a sequence as in lemma 1.4.1 for an admissible metric of type $\lambda^{-1}(X) \Gamma$, where $\lambda$ is a $\Gamma$-weight and $\Gamma=\Gamma^{\sigma}$. Let $\omega$ be a locally bounded function such that $|\omega(X)| \leq M(X)$ where $M$ is a $\Gamma$-weight. Assume that, for each $k$, there exist a bounded function $\omega_{k}$ such that $\omega(X)=\omega_{k}(X)$ for all $X \in \operatorname{supp} \chi_{k}$ and such that for all $X \in \mathbb{R}^{2 n}$, $\left|\omega_{k}(X)\right| \leq M(X) \lambda(X)^{N_{0}}$. Then with $\widetilde{\omega}(X)=\int \omega(Y) 2^{n} \exp -2 \pi \Gamma(X-Y) d Y$, we have

$$
\chi_{k}(X) \widetilde{\omega}(X)=\chi_{k}(X) \widetilde{\omega_{k}}(X)+r_{k}(X), \sum_{k} r_{k} \in S\left(\lambda^{-\infty}, \Gamma\right) .
$$


Proof. - We already know from the lemma A.1.3 that $X \mapsto \widetilde{\omega}(X)=$ $\int \omega(Y) 2^{n} \exp -2 \pi \Gamma(X-Y) d Y$ belongs to $S(M, \Gamma)$. We check now

$$
\begin{aligned}
& =\chi_{k}(X) \int \psi_{k}(Y) \omega(Y) 2^{n} \exp -2 \pi \Gamma(X-Y) d Y \\
& \quad+\chi_{k}(X) \int_{Y, \psi_{k}(Y) \neq 1}\left(1-\psi_{k}(Y)\right) \omega(Y) 2^{n} \exp -2 \pi \Gamma(X-Y) d Y \\
& =\chi_{k}(X) \int \psi_{k}(Y) \omega_{k}(Y) 2^{n} \exp -2 \pi \Gamma(X-Y) d Y+r_{k}(X) .
\end{aligned}
$$

We have $\Gamma\left(U_{k}-\left(U_{k}^{*}\right)^{c}\right)=\inf _{\Gamma(T)<1 \leq \Gamma(S)} \Gamma\left(X_{k}+r_{0} \lambda\left(X_{k}\right)^{1 / 2} T-X_{k}-\right.$ $\left.\lambda\left(X_{k}\right)^{1 / 2} 2 r_{0} S\right)$ and thus $\Gamma\left(U_{k}-\left(U_{k}^{*}\right)^{c}\right) \geq \lambda\left(X_{k}\right) r_{0}^{2}$. Since $\psi_{k}$ is equal to 1 on $U_{k}^{*}$ (notations of section 1.4) we obtain from (A.1.15)

$$
\left|r_{k}^{(j)}(X)\right|_{\Gamma} \leq C_{j} \psi_{k}(X) \exp -\pi \Gamma\left(U_{k}-\left(U_{k}^{*}\right)^{c}\right) \leq C_{j, N, r_{0}} \psi_{k}(X) \lambda(X)^{-N}
$$

and thus $\sum_{k} r_{k} \in S\left(\lambda^{-\infty}, \Gamma\right)$. We obtain

$$
\begin{aligned}
\chi_{k} \widetilde{\omega}=\chi_{k}\left(\psi_{k} \omega_{k}\right. & \left.* 2^{n} \exp -2 \pi \Gamma\right)+r_{k} \\
& =\chi_{k}\left(\omega_{k} * 2^{n} \exp -2 \pi \Gamma\right)+\chi_{k}\left(\omega_{k}\left(\psi_{k}-1\right) * 2^{n} \exp -2 \pi \Gamma\right)+r_{k},
\end{aligned}
$$

and applying again the same reasoning to the penultimate term above, we get for $Y \in\left(U_{k}^{*}\right)^{c}$ and $X \in U_{k}$, that $\Gamma(X-Y) \geq \lambda\left(X_{k}\right) r_{0}^{2}$ the following estimate for the integrand

$$
\begin{aligned}
\exp & -\pi \Gamma(X-Y) \exp -\pi \lambda\left(X_{k}\right) r_{0}^{2} \times M(Y) \lambda(Y)^{N_{0}} \\
& \leq C M(X) \lambda(X)^{N_{0}}(1+\Gamma(X-Y))^{N_{0}} \exp -\pi \Gamma(X-Y) \exp -\pi \lambda\left(X_{k}\right) r_{0}^{2} \\
& \leq C^{\prime} M(X) \lambda\left(X_{k}\right)^{N_{0}}\left(1+\Gamma\left(X-X_{k}\right)\right)^{N_{0}} \exp -\frac{\pi}{2} \Gamma(X-Y) \exp -\pi \lambda\left(X_{k}\right) r_{0}^{2} \\
& \leq C^{\prime \prime} M(X) \lambda\left(X_{k}\right)^{3 N_{0}} \exp -\frac{\pi}{2} \Gamma(X-Y) \exp -\pi \lambda\left(X_{k}\right) r_{0}^{2} \\
& \leq C^{\prime \prime \prime} M(X) \lambda\left(X_{k}\right)^{3 N_{0}} \exp -\frac{\pi}{2} \Gamma(X-Y) \exp -\pi \lambda\left(X_{k}\right) r_{0}^{2}
\end{aligned}
$$

which yields the result.

Definition A.1.7. - Let $\Gamma$ be a symplectic quadratic form on $\mathbb{R}^{n} \times \mathbb{R}^{n}$, i.e., a positive definite quadratic form such that $\Gamma=\Gamma^{\sigma}$ (see definition 1.3.2(2)). There exists a unique linear symplectic mapping $A$ such that for all $X=(x, \xi)$, $\Gamma(A X)=\sum_{1 \leq j \leq n} x_{j}^{2}+\xi_{j}^{2}$. Let $U$ be a metaplectic transformation in the fiber of $A$. Then for $a \in L^{\infty}\left(\mathbb{R}^{2 n}\right)$, we define

$$
a^{\mathrm{Wick}(\Gamma)}=\int a(Y) 2^{n}(\exp -2 \pi \Gamma(\cdot-Y))^{w} d Y=U(a \circ A)^{\mathrm{Wick}} U^{*}
$$


REMARK A.1.8. - Note that since $U$ is uniquely determined up to a factor of modulus one, that definition is consistent. We remark also that, defining for $X \in \mathbb{R}^{2 n}, \Phi(X)=2^{n} \exp -2 \pi \Gamma(X)$, we have $\Phi(A X-A Y)=2^{n} \exp -2 \pi \mid X-$ $\left.Y\right|^{2}$, which is the Weyl symbol of $\Sigma_{Y}$ (definition A.1.1). From the Segal formula, we have, with a metaplectic $U$ in the fiber of $A$

$$
\Phi(X-Z)^{w}=U \Phi(A X-Z)^{w} U^{*}
$$

and thus we can justify the equality in formula (A.1.16) since

$$
\begin{aligned}
\int a(Y) 2^{n}(\exp -2 \pi \Gamma(X-Y))^{w} d Y & =\int a(A Y) \Phi(X-A Y)^{w} d Y= \\
\int a(A Y) U \Phi(A X-A Y)^{w} U^{*} & =\int a(A Y) U \Sigma_{Y} U^{*} d Y=U(a \circ A)^{\mathrm{Wick}} U^{*} .
\end{aligned}
$$

REMARK A.1.9. - We can also notice that the definition above is consistent with the fact that Wick and Weyl quantization coincide for linear forms: if $a$ is a linear form, we have

$$
a^{\mathrm{Wick}(\Gamma)}=U(a \circ A)^{\mathrm{Wick}} U^{*}=U(a \circ A)^{w} U^{*}=U U^{*} a^{w} U U^{*}=a^{w} .
$$

Also, it is easy with the formula (A.1.16) to check that the results of section A.1 on the Wick quantization can be extended, mutatis mutandis, to the Wick $(\Gamma)$ quantization.

A.2. Properties of some metrics. Proof of the remark following definition 1.3.1. - Using a partition of unity related to the slowly varying $g$, as in [3], we define $M_{*}(X)=\int_{\mathbb{R}^{2 n}} M(Y) \varphi_{Y}(X)\left|g_{Y}\right|^{1 / 2} d Y$. It is a simple matter left to the reader to check that $M_{*}$ belongs to $S(M, g)$ and satisfies (1.3.3).

LEMMA A.2.1. - Let $\Gamma$ be a positive definite quadratic form on $\mathbb{R}^{2 n}$ such that $\Gamma=\Gamma^{\sigma}$ and let $g_{X}=\lambda(X)^{-1} \Gamma$ be a metric conformal to $\Gamma$ such that $g$ is slowly varying and $\inf _{X} \lambda(X) \geq 1$. Then the metric $g$ satisfies $g_{X}(T) \leq$ $C g_{Y}(T)(1+\Gamma(X-Y))$, i.e.,

$$
\frac{\lambda(Y)}{\lambda(X)} \leq C(1+\Gamma(X-Y))
$$

implying that $g$ is admissible.

Proof. - Since $g$ is slowly varying, we may assume, with a positive $r_{0}, g_{Y}(Y-$ $X) \geq r_{0}^{2}$, which means $\Gamma(Y-X) \geq r_{0}^{2} \lambda(Y)$ and using $\lambda(X) \geq 1$ we get $\lambda(Y) / \lambda(X) \leq r_{0}^{-2} \Gamma(Y-X)$. 
LEMMA A.2.2. - Let $\Gamma$ be a positive definite quadratic form on $\mathbb{R}^{2 n}$ such that $\Gamma=\Gamma^{\sigma}$ and let $g_{X}=\lambda(X)^{-1} \Gamma$ be a metric conformal to $\Gamma$. Assume that $\lambda(X)=d(X)^{2}+\lambda_{1}(X)$ with a function $d$ uniformly Lipschitz continuous (with respect to $\Gamma$ ) and $\lambda_{1}^{-1} \Gamma$ slowly varying with $\lambda_{1} \geq 1$. Then the metric $g$ is slowly varying.

Proof. - Let us assume that $|X-Y|^{2} \leq r^{2}\left(d(X)^{2}+\lambda_{1}(X)\right)$. If $d(X)^{2} \leq$ $\lambda_{1}(X)$, using the fact that $\lambda_{1}^{-1} \Gamma$ is slowly varying, we can choose $r$ small enough so that $\lambda_{1}(X) \leq C_{1} \lambda_{1}(Y)$ and thus

$$
\lambda(X) \leq 2 C_{1} \lambda_{1}(Y) \leq 2 C_{1} \lambda(Y) .
$$

If $d(X)^{2}>\lambda_{1}(X)$, we have, with $L$ standing for the Lipschitz constant of $d$,

$$
2^{-1 / 2} \lambda(X)^{1 / 2}<d(X) \leq d(Y)+L|X-Y| \leq \lambda(Y)^{1 / 2}+\operatorname{Lr} \lambda(X)^{1 / 2}
$$

so that, for $r \leq \frac{1}{2^{3 / 2} L+1}$ we get $\lambda(X) \leq 8 \lambda(Y)$.

REMARK A.2.3. - It is a simple exercise left to the reader to show that (1) in Definition 1.3.1 is satisfied whenever there exists $r_{0}>0, C_{0}>0$ such that for all $X, Y, T \in \mathbb{R}^{2 n}, g_{X}(Y-X) \leq r_{0}^{2}$ implies $g_{Y}(T) \leq C_{0} g_{X}(T)$.

Taking this remark into account, we complete the proof of the lemma.

A.3. Proof of Lemma 2.1.5 on the proper class. - All norms in this proof are taken with respect to the constant quadratic form $\Gamma$, so we omit the index everywhere and denote $\|\cdot\|_{\Gamma}$ by $|\cdot|$. Since for all $j \in \mathbb{N},\left|f^{(j)}(X)\right| \leq$ $\gamma_{j} \Lambda^{m-\frac{j}{2}}$, we get $1 \leq \lambda(X) \leq 1+\Lambda \max _{\substack{0 \leq j<2 m \\ j \in \mathbb{N}}} \gamma_{j}^{\frac{2}{2 m-j}}=1+\gamma \Lambda \leq(1+\gamma) \Lambda$ and (2.1.12). For $0 \leq j<2 m$, we have from the definition of $\lambda$, the estimate $\left|f^{(j)}(X)\right| \leq \lambda(X)^{m-\frac{j}{2}}$, and for $j \geq 2 m$, we can use

$$
\left|f^{(j)}(X)\right| \leq \gamma_{j} \Lambda^{m-\frac{j}{2}}=\gamma_{j} \Lambda^{-\frac{(j-2 m)}{2}} \leq \gamma_{j} \lambda^{-\frac{(j-2 m)}{2}}(1+\gamma)^{\frac{(j-2 m)}{2}},
$$

so that $f \in S\left(\lambda^{m}, \lambda^{-1} \Gamma\right)$ with a $j$-th semi-norm less than 1 for $j<2 m$ and less than $\gamma_{j}(1+\gamma)^{\frac{(j-2 m)}{2}}$ for $j \geq 2 m$.

Let us now prove that $\lambda^{-1} \Gamma$ is slowly varying. Let us assume that $|X-Y|^{2} \leq$ $r^{2} \lambda(X)$. Using Taylor's formula, we get for the smallest integer $N \geq 2 m$ $(N=-[-2 m])$ and $0 \leq j<2 m$,

$$
\left|f^{(j)}(X)\right| \leq \sum_{l, j+l<2 m}\left|f^{(j+l)}(Y)\right| \frac{r^{l}}{l !} \lambda(X)^{l / 2}+\gamma_{N} \Lambda^{m-\frac{N}{2}} \frac{r^{N-j}}{(N-j) !} \lambda(X)^{(N-j) / 2},
$$

BULlETIN DE LA SOCIÉtÉ MATHÉMATIQUE DE FRANCE 
so that $\left|f^{(j)}(X)\right| \leq \sum_{l, j+l<2 m} \lambda(Y)^{\frac{2 m-j-l}{2}} \lambda(X)^{\frac{l}{2}} \frac{r^{l}}{l !}+\gamma_{N} \Lambda^{\frac{2 m-N}{2}} \lambda(X)^{\frac{N-j}{2}} \frac{r^{N-j}}{(N-j) !}$, and

$$
\begin{aligned}
& \left|f^{(j)}(X)\right| \\
& \leq \sum_{l, j+l<2 m}\left(\lambda(Y)^{\frac{2 m-j}{2}}\right)^{\frac{2 m-j-l}{2 m-j}}\left(\lambda(X)^{\frac{2 m-j}{2}}\right)^{\frac{l}{2 m-j}} \frac{r^{l}}{l !}+\gamma_{N} \Lambda^{\frac{2 m-N}{2}} \lambda(X)^{\frac{N-j}{2}} \frac{r^{N-j}}{(N-j) !} \\
& \leq \sum_{l, j+l<2 m} \frac{2 m-j-l}{2 m-j} \lambda(Y)^{\frac{2 m-j}{2}} \frac{r^{l}}{l !}+\frac{l}{2 m-j} \lambda(X)^{\frac{2 m-j}{2}} \frac{r^{l}}{l !} \\
& \quad+\gamma_{N}(1+\gamma)^{\frac{N-2 m}{2}} \lambda(X)^{\frac{2 m-j}{2}} \frac{r^{N-j}}{(N-j) !}
\end{aligned}
$$

implying

$$
\begin{aligned}
\left|f^{(j)}(X)\right| \leq \lambda(Y)^{\frac{2 m-j}{2}} & \overbrace{\sum_{l, j+l<2 m} \frac{2 m-j-l}{2 m-j} \frac{r^{l}}{l !}}^{\text {a polynomial in } r} \\
& +\lambda(X)^{\frac{2 m-j}{2}} \underbrace{\left(\sum_{1 \leq l, j+l<2 m} \frac{l}{2 m-j} \frac{r^{l}}{l !}+\gamma_{N}(1+\gamma)^{\frac{N-2 m}{2}} \frac{r^{N-j}}{(N-j) !}\right)}_{=\epsilon(r) \text { goes to zero with } r .} .
\end{aligned}
$$

Assuming then that $j$ was chosen so that $\lambda(X)=1+\left|f^{(j)}(X)\right|^{\frac{2}{2 m-j}}$, we get

$$
\lambda(X) \leq 1+\left(\lambda(Y)^{\frac{2 m-j}{2}} p(r)+\lambda(X)^{\frac{2 m-j}{2}} \epsilon(r)\right)^{\frac{2}{2 m-j}},
$$

so that there exist $r_{0}>0, C_{0} \geq 1$, depending only on the $N$ first semi-norms of $f$, such that for $r \leq r_{0}$, we have

$$
|X-Y|^{2} \leq r^{2} \lambda(X) \Longrightarrow \lambda(X) \leq C_{0} \lambda(Y)
$$

and thus $r \leq r_{0},|X-Y|^{2} \leq r^{2} C_{0}^{-1} \lambda(X) \Longrightarrow C_{0}^{-1} \lambda(X) \leq \lambda(Y) \leq C_{0} \lambda(X)$, which is the property (1) in Definition 1.3.1. The property (2) in that definition is obviously satisfied since $\lambda(X) \geq 1$. Moreover, we get a stronger property than (3) from the Lemma A.2.1 above in this appendix.

A.4. Some a priori estimates and loss of derivatives. - In this section, we prove that when a factorization occurs in an abstract setting, it is possible to limit the loss of derivatives to $3 / 2$ (the loss is always counted with respect to the elliptic case). Let us study the model-case

$$
L=D_{t}+i A_{0} B_{1}, \quad A_{0} \in \mathrm{Op}\left(S^{0}\right), B_{1} \in \mathrm{Op}\left(S^{1}\right)
$$

TOME $134-2006-\mathrm{N}^{\mathrm{O}} 4$ 
with real-valued Weyl symbols such that $A_{0} \geq c_{0} \Lambda^{-1}, \dot{B_{1}} \geq 0$. We compute, using the notation

$$
\begin{aligned}
& \|u\|=\left(\int|u(t)|^{2} d t\right)^{1 / 2}, \quad|v|=\|v\|_{\mathbb{H}}, \quad \mathbb{H}=L^{2}\left(\mathbb{R}^{n}\right), \quad|u|_{\infty}=\sup _{t \in \mathbb{R}}|u(t)|, \\
& 2 \operatorname{Re}\left\langle L u, i B_{1} u\right\rangle=\left\langle\dot{B}_{1}(t) u(t), u(t)\right\rangle+2 \operatorname{Re}\left\langle A_{0} B_{1} u, B_{1} u\right\rangle \geq 2 c_{0} \Lambda^{-1}\left\|B_{1} u\right\|^{2} .
\end{aligned}
$$

As a consequence, for $\operatorname{supp} u \subset[-T, T]$,

$2 \operatorname{Re}\left\langle L u, i B_{1} u\right\rangle+2 \operatorname{Re}\left\langle L u, i H\left(t-T_{0}\right) u\right\rangle$

$$
\begin{aligned}
& \geq c_{0} \Lambda^{-1}\left\|B_{1} u\right\|^{2}+|u|_{\infty}^{2}+\left\|A_{0}^{1 / 2} B_{1} u\right\|^{2}+2 \operatorname{Re}\left\langle A_{0}^{1 / 2} B_{1} u, i H_{T_{0}} A_{0}^{1 / 2} u\right\rangle \\
& \geq c_{0} \Lambda^{-1}\left\|B_{1} u\right\|^{2}+|u|_{\infty}^{2}\left(1-\sup _{|t| \leq T}\left\|A_{0}(t)\right\| T\right) \\
& \quad \text { (for T small enough) } \geq c_{0} \Lambda^{-1}\left\|B_{1} u\right\|^{2}+\frac{1}{2}|u|_{\infty}^{2},
\end{aligned}
$$

so that $c_{0}^{-1} \Lambda\|L u\|^{2}+c_{0} \Lambda^{-1}\left\|B_{1} u\right\|^{2}+2\|L u\|\|u\| \geq c_{0} \Lambda^{-1}\left\|B_{1} u\right\|^{2}+\frac{1}{2}|u|_{\infty}^{2}$ and thus

$$
\left(c_{0}^{-1} \Lambda+1\right)\|L u\|^{2}+T|u|_{\infty}^{2} \geq \frac{1}{2}|u|_{\infty}^{2}
$$

entailing for $T \leq 1 / 4, \quad\left(c_{0}^{-1} \Lambda+1\right)\|L u\|^{2} \geq \frac{1}{4}|u|_{\infty}^{2}$, which gives $\|L u\| \gg$ $\Lambda^{-1 / 2}\|u\|$, an estimate with loss of $3 / 2$ derivatives.

The next question is obviously: how do we manage to get the estimate $A_{0} \geq \Lambda^{-1}$ ? Assuming $A_{0} \geq-C \Lambda^{-1}$, we can always consider instead $A_{0}+(C+$ 1) $\Lambda^{-1} \geq \Lambda^{-1}$; now this modifies the operator $L$ and although our estimate is too weak to absorb a zeroth order perturbation, it is enough to check that the energy method is stable by zeroth order perturbation. We consider then

$$
D_{t}+i A_{0} B_{1}+S+i R, \quad A_{0} \geq \Lambda^{-1}, \quad S, R \in \mathrm{Op}\left(S^{0}\right) .
$$

Inspecting the method above, we see that $S$ will not produce any trouble, since we shall commute it with $B_{1}$, producing an operator of order 0 . The term produced by $R$ are more delicate to handle: we shall have to deal with

$$
2\left\langle R u, B_{1} u\right\rangle+2\left\langle R u, H_{T_{0}} u\right\rangle .
$$

The second term is $L^{2}$ bounded and can be absorbed. There is no simple way to absorb the first term, which is of size $\left\|B_{1} u\right\|\|u\|$ which is too large with respect to the terms that we dominate. However we can consider the $L^{2}$-bounded invertible operator $U(t)$ (which is in $\mathrm{Op}\left(S^{0}\right)$ and self-adjoint) such that $U(0)=\mathrm{Id}$ and $\dot{U}(t)=-U(t) R(t)$ so that

$$
\begin{aligned}
L=D_{t}+i R+i A_{0} B_{1}+S=U(t)^{-1} D_{t} U(t)+i A_{0} B_{1}+S & \\
=U(t)^{-1} & \left(D_{t}+i A_{0} B_{1}+S\right) U(t)-U(t)^{-1}\left[i A_{0} B_{1}+S, U(t)\right] \\
& =U(t)^{-1}\left(D_{t}+i A_{0} B_{1}+S+\left[U(t), i A_{0} B_{1}+S\right] U(t)^{-1}\right) U(t) .
\end{aligned}
$$

BULLETIN DE LA SOCIÉtÉ MATHÉMATIQUE DE FRANCE 
Now the term $\left[U(t), i A_{0} B_{1}\right] U(t)^{-1}$ has a real-valued principal symbol in $S^{0}$ and amounts to a modification of $\mathrm{S}$, up to unimportant terms of order -1 . The term $[U(t), S] U(t)^{-1}$ is of order -1 and can be absorbed. We have proven the following lemma.

LEMMA A.4.1. - Let $\Lambda \geq 1$ be given. We consider the metric $G=|d x|^{2}+$ $\Lambda^{-2}|d \xi|^{2}$ on $\mathbb{R}^{n} \times \mathbb{R}^{n}$. Let $a_{0}(t, x, \xi)$ be in $S(1, G)$ such that $a_{0}(t, x, \xi) \geq 0$. Let $b_{1}(t, x, \xi)$ be real-valued and in $S(\Lambda, G)$ such that

$$
\left(b_{1}(t, x, \xi)-b(s, x, \xi)\right)(t-s) \geq 0 .
$$

Let $r(t, x, \xi)$ be a complex-valued symbol in $S(1, G)$. Assuming that $a_{0}, b_{1}, r_{0}$ are continuous functions, there exists a constant $C>0$ depending only on the semi-norms of the symbols $a_{0}, b_{1}, r_{0}$, such that, for all $u \in C_{c}^{1}\left([-T, T], L^{2}\left(\mathbb{R}^{n}\right)\right)$ with $C T \leq 1$,

$$
C\|L u\|_{L^{2}\left(\mathbb{R}^{n+1}\right)} \geq \Lambda^{-1 / 2} T^{-1}\|u\|_{L^{2}\left(\mathbb{R}^{n+1}\right)} .
$$

A.5. Some lemmas on symbolic calculus. - Let $g$ be an admissible metric on $\mathbb{R}^{2 n}$ and $m$ be a $g$-weight (see definition 1.3.1). Then, at each point $X \in \mathbb{R}^{2 n}$, we can define a metric $g_{X}^{\sharp}$ by taking the geometric mean of $g_{X}, g_{X}^{\sigma}$ so that in particular

$$
g_{X} \leq g_{X}^{\sharp}=\left(g_{X}^{\sharp}\right)^{\sigma} \leq g_{X}^{\sigma} .
$$

We define

$$
h(X)=\sup _{g_{X}^{\sharp}(T)=1} g_{X}(T)
$$

and we note that whenever $g^{\sigma}=\lambda^{2} g$ we get from the definition 1.3.1 that $g^{\sharp}=\lambda_{g} g$ and $\lambda_{g}=1 / h$.

Definition A.5.1. - Let $l$ be a nonnegative integer. We define the set $S_{l}(m, g)$ as the set of smooth functions $a$ defined on $\mathbb{R}^{2 n}$ such that $a$ satisfies the estimates of $S(m, g)$ for derivatives of order $\leq l$, and the estimates of $S\left(m, g^{\sharp}\right)$ for derivatives of order $\geq l+1$, which means

$$
\left|a^{(k)}(X) T^{k}\right| \leq C_{k} m(X) \times \begin{cases}g_{X}(T)^{k / 2} & \text { if } k \leq l, \\ g_{X}^{\sharp}(T)^{k / 2} h(X)^{\frac{l+1}{2}} & \text { if } k \geq l+1, \\ & \text { with } h(X)=\sup _{g_{X}^{\sharp}(T)=1} g_{X}(T) .\end{cases}
$$

Note that since $h \leq 1$ and $g \leq h g^{\sharp}$, we get $S(m, g) \subset S_{l}(m, g)$. If $g=$ $\lambda(X)^{-1} \Gamma_{X}$, where $\lambda(X)$ is positive (scalar) and $\Gamma_{X}=\Gamma_{X}^{\sigma}$, then $g_{X}^{\sharp}=\Gamma_{X}$ and $a$ belongs to $S_{l}\left(m, \lambda^{-1} \Gamma\right)$ means

$$
\left|a^{(k)}(X)\right|_{\Gamma_{X}} \leq C_{k} m(X) \times \begin{cases}\lambda(X)^{-k / 2} & \text { if } k \leq l, \\ \lambda(X)^{-l / 2} & \text { if } k \geq l+1 .\end{cases}
$$

Moreover, if $g \equiv g^{\sharp}$, then for all $l, S(m, g)=S_{l}(m, g)$. 
LEMmA A.5.2. - Let $\Gamma$ be a positive definite quadratic form on $\mathbb{R}^{2 n}$ such that $\Gamma=\Gamma^{\sigma}$ and $\lambda$ be a $\Gamma$-weight. Let $b$ be a symbol in $S_{1}\left(\lambda^{m}, \lambda^{-1} \Gamma\right)$, where $m$ is a real number. Then $b \sharp b-b^{2} \in S\left(\lambda^{2 m-1}, \Gamma\right)$

Proof. - We have $(b \sharp b)(X)=\exp i \pi\left[D_{X_{1}}, D_{X_{2}}\right]\left(b\left(X_{1}\right) \otimes b\left(X_{2}\right)\right)_{\mid X_{1}=X_{2}=X}$ so that using Taylor's formula with integral remainder for $s \mapsto e^{s}$ yields

$(b \sharp b)(X)=b(X)^{2}+\int_{0}^{1} \exp i \pi \theta\left[D_{X_{1}}, D_{X_{2}}\right] d \theta i \pi\left[D_{X_{1}}, D_{X_{2}}\right] b\left(X_{1}\right) \otimes b\left(X_{2}\right)_{\mid X_{1}=X_{2}=X}$.

Since $b^{\prime} \in S\left(\lambda^{m-1 / 2}, \Gamma\right)$ and

$$
\begin{aligned}
& \exp i \pi \theta\left[D_{X_{1}}, D_{X_{2}}\right]\left(a_{1}\left(X_{1}\right) \otimes a_{2}\left(X_{2}\right)\right) \\
& =\exp i \pi\left[D_{X_{1} \theta^{-1 / 2}}, D_{X_{2} \theta^{-1 / 2}}\right]\left(a_{1}\left(\theta^{-1 / 2} X_{1} \theta^{1 / 2}\right) \otimes a_{2}\left(\theta^{-1 / 2} X_{2} \theta^{1 / 2}\right)\right)_{\mid X_{1}=X_{2}=X} \\
& =\exp i \pi\left[D_{Y_{1}}, D_{Y_{2}}\right]\left(a_{1}\left(\theta^{1 / 2} Y_{1}\right) \otimes a_{2}\left(\theta^{1 / 2} Y_{2}\right)\right)_{\mid Y_{1}=Y_{2}=\theta^{-1 / 2} X} \\
& =\left(\left(a_{1} \circ \theta^{1 / 2}\right) \sharp\left(a_{2} \circ \theta^{1 / 2}\right)\right)\left(\theta^{-1 / 2} X\right),
\end{aligned}
$$

we get that, if $a_{j} \in S\left(\lambda^{m_{j}}, \Gamma\right)$, we have $a_{j} \circ \theta^{1 / 2} \in S\left(\lambda^{m_{j}}, \theta \Gamma\right)$ so that the symbolic calculus for the metric $\theta \Gamma$ (observe that it is admissible for $\theta$ bounded) gives

$$
\left(a_{1} \circ \theta^{1 / 2}\right) \sharp\left(a_{2} \circ \theta^{1 / 2}\right) \in S\left(\lambda^{m_{1}+m_{2}}, \theta \Gamma\right)
$$

which implies $\left(\left(a_{1} \circ \theta^{1 / 2}\right) \sharp\left(a_{2} \circ \theta^{1 / 2}\right)\right) \circ \theta^{-1 / 2} \in S\left(\lambda^{m_{1}+m_{2}}, \Gamma\right)$. Applying this to the integral above gives the result of the lemma.

LEMMA A.5.3. - Let $\Gamma$ be a positive definite quadratic form on $\mathbb{R}^{2 n}$ such that $\Gamma=\Gamma^{\sigma}$ and $\lambda$ be a $\Gamma$-weight. Let $l \in \mathbb{N}, \mu \in \mathbb{R}$ and a be a locally bounded function defined on $\mathbb{R}^{2 n}$ such that

$$
\forall j \in\{0, \ldots, l\}, \quad\left|a^{(j)}(X)\right| \leq C \lambda(X)^{\mu-\frac{j}{2}} .
$$

Then the function $a * \exp -2 \pi \Gamma$ belongs to $S_{l}\left(\lambda^{\mu}, \lambda^{-1} \Gamma\right)$.

Proof. - We use the formula $(a * \exp -2 \pi \Gamma)(X)=\int a(X-Y) \exp -2 \pi \Gamma(Y) d Y$ to obtain the estimate for the derivatives of order $\leq l$ : we get for $k \leq l$

$$
\begin{aligned}
& \left|(a * \exp -2 \pi \Gamma)^{(k)}(X)\right| \leq C \lambda(X)^{\mu-\frac{k}{2}} \int \frac{\lambda(X-Y)^{\mu-\frac{k}{2}}}{\lambda(X)^{\mu-\frac{k}{2}}} \exp -2 \pi \Gamma(Y) d Y \\
& \leq C \lambda(X)^{\mu-\frac{k}{2}} \int(1+\Gamma(Y))^{N\left|\mu-\frac{k}{2}\right|} \exp -2 \pi \Gamma(Y) d Y=C^{\prime} \lambda(X)^{\mu-\frac{k}{2}},
\end{aligned}
$$

and for $k>l$ we have $(a * \exp -2 \pi \Gamma)^{(k)}=\left(a^{(l)} *(\exp -2 \pi \Gamma)^{(k-l)}\right)$ yielding immediately the result.

BULLETIN DE LA SOCIÉtÉ MATHÉMATIQUE DE FRANCE 
Let us recall the composition formula in the Weyl quantization, with the symplectic form [, ] given in (1.3.2). We have $a^{w} b^{w}=(a \sharp b)^{w}$ and, for $X \in \mathbb{R}^{2 n}$,

$$
\begin{aligned}
(a \sharp b)(X) & =2^{2 n} \iint_{\mathbb{R}^{2 n} \times \mathbb{R}^{2 n}} a(Y) b(Z) \exp -4 i \pi[X-Y, X-Z] d Y d Z \\
& =2^{2 n} \iint_{\mathbb{R}^{2 n} \times \mathbb{R}^{2 n}} a(Y+X) b(Z+X) \exp -4 i \pi[Y, Z] d Y d Z .
\end{aligned}
$$

We note also that

$$
(a \sharp b)^{\prime}=a^{\prime} \sharp b+a \sharp b^{\prime} .
$$

Moreover, if $a$ is a function only of $\xi$, we have

$$
\begin{aligned}
& (a \sharp b)(x, \xi)=2^{2 n} \int_{\mathbb{R}^{4 n}} a(\eta) b(z, \zeta) e^{-4 i \pi(\xi-\eta)(x-z)} e^{4 i \pi(x-y)(\xi-\zeta)} d y d \eta d z d \zeta \\
& =2^{n} \int_{\mathbb{R}^{2 n}} a(\eta) b(z, \xi) e^{-4 i \pi(\xi-\eta)(x-z)} d \eta d z \\
& =2^{n} \int_{\mathbb{R}^{2 n}}\left(\left(1+D_{\eta}^{2} / 4\right)^{N} a\right)(\eta) b(z, \xi)\left(1+|x-z|^{2}\right)^{-N} e^{-4 i \pi(\xi-\eta)(x-z)} d \eta d z \\
& =2^{n} \int_{\mathbb{R}^{2 n}}\left(\left(1+D_{\eta}^{2} / 4\right)^{N} a\right)(\eta)\left(1+|\xi-\eta|^{2}\right)^{-N}\left(1+D_{z}^{2} / 4\right)^{N} \\
& \quad\left(b(z, \xi)\left(1+|x-z|^{2}\right)^{-N}\right) e^{-4 i \pi(\xi-\eta)(x-z)} d \eta d z
\end{aligned}
$$

so that with $N \geq E(n / 2)+1$

$$
|(a \sharp b)(x, \xi)| \leq \max _{j \leq 2 N}\left\|a^{(j)}\right\|_{L^{\infty}} \max _{j \leq 2 N}\left\|b^{(j)}\right\|_{L^{\infty}}(1+|\xi-\operatorname{supp} a|)^{-N / 2} c(n, N) .
$$

\section{A.6. The Beals-Fefferman reduction}

LemmA A.6.1. - Let $F: \mathbb{R} \rightarrow \mathbb{R}$ be a $C^{2}$ function such that

$$
16|F(0)|<F^{\prime}(0)^{2}, \quad\left\|F^{\prime \prime}\right\|_{L^{\infty}(\mathbb{R})} \leq 1 .
$$

We set $\rho=\left|F^{\prime}(0)\right| / 4$. Then there exists $t_{0} \in[-\rho / 2, \rho / 2]$ and $e \in C^{1}(\mathbb{R})$ such that

(A.6.2) for $|t| \leq \rho, F(t)=\left(t-t_{0}\right) e(t), \quad 8 \rho \geq e(t) \geq \rho, \quad\left\|e^{\prime}\right\|_{L^{\infty}(\mathbb{R})} \leq 1$.

Proof. - Assume first that $F(0)=0$ and $F^{\prime}(0)=4 \rho$. Then, for $|t| \leq 2 \rho$,

$$
F(t)=t e(t), \quad 6 \rho \geq e(t) \geq 4 \rho-2 \rho=2 \rho,\left\|e^{\prime}\right\|_{L^{\infty}(\mathbb{R})} \leq 1 .
$$

Now if $F(0)>0$ and $F^{\prime}(0)=4 \rho, F\left(-\frac{\rho}{2}\right) \leq \rho^{2}-\frac{\rho}{2} 4 \rho+\frac{\rho^{2}}{4}<0$, so that, for some $\left.t_{0} \in\right]-\rho / 2,0\left[\right.$ we have $F\left(t_{0}\right)=0$. Using what was done above, we have for $|s| \leq\left|F^{\prime}\left(t_{0}\right)\right| / 2$,

$F\left(s+t_{0}\right)=\left(s+t_{0}\right) e_{0}(s), \quad 3\left|F^{\prime}\left(t_{0}\right)\right| / 2 \geq e_{0}(s) \geq\left|F^{\prime}\left(t_{0}\right)\right| / 2, \quad\left\|e_{0}^{\prime}\right\|_{L^{\infty}(\mathbb{R})} \leq 1$.

TOME $134-2006-\mathrm{N}^{\mathrm{O}} 4$ 
But since

$$
\frac{\left|F^{\prime}\left(t_{0}\right)\right|}{2} \geq \frac{1}{2}\left(4 \rho-\frac{\rho}{2}\right)=\frac{7 \rho}{4} \quad \text { and } \quad \frac{7 \rho}{4}-\frac{\rho}{2}=\frac{5 \rho}{4} \geq \rho
$$

we have on $\left[t_{0}-\frac{7 \rho}{4}, t_{0}+\frac{7 \rho}{4}\right]$ which contains $[-\rho, \rho]$,

$F(t)=\left(t-t_{0}\right) e(t),\left|t_{0}\right| \leq \rho / 2,8 \rho \geq \frac{27 \rho}{4} \geq e(t) \geq 7 \rho / 4 \geq \rho,\left\|e^{\prime}\right\|_{L^{\infty}(\mathbb{R})} \leq 1$.

LEMMA A.6.2. - Let $F: \mathbb{R}^{d} \rightarrow \mathbb{R}$ be a $C^{2}$ function such that

(A.6.3) $\quad 2^{6}|F(0)|<\|\nabla F(0)\|^{2}, \quad\left\|F^{\prime \prime}\right\|_{L^{\infty}\left(\mathbb{R}^{d}\right)} \leq 1$.

We set $\rho=\|\nabla F(0)\| 2^{-5}$. There exists two $C^{1}$ functions $\alpha: \mathbb{R}^{d-1} \rightarrow$ $[-5 \rho, 5 \rho]$ and $e: \mathbb{R}^{d} \rightarrow[7 \rho, 70 \rho]$, a set of orthonormal coordinates $\left(x_{1}, x^{\prime}\right) \in \mathbb{R} \times \mathbb{R}^{d-1}$ such that for $\max \left(\left|x_{1}\right|,\left|x^{\prime}\right|\right) \leq \rho$,

$$
F(x)=\left(x_{1}+\alpha\left(x^{\prime}\right)\right) e(x), \quad\left\|e^{\prime}\right\|_{L^{\infty}\left(\mathbb{R}^{d}\right)} \leq 1, \quad\left\|\alpha^{\prime}\right\|_{L^{\infty}\left(\mathbb{R}^{d-1}\right)} \leq 1 .
$$

Proof. - We can choose the coordinates so that $\nabla F(0)=\frac{\partial F}{\partial x_{1}}(0) \overrightarrow{e_{1}}$. Then for $\left|x^{\prime}\right| \leq \rho$, we have $\left|F\left(0, x^{\prime}\right)\right| \leq 2^{-6+10} \rho^{2}+\rho 2^{5} \rho+\frac{1}{2} \rho^{2}=\rho^{2}\left(2^{5}+2^{4}+2^{-1}\right)$ and

so that

$$
\left|\frac{\partial F}{\partial x_{1}}\left(0, x^{\prime}\right)\right| \geq\left|\frac{\partial F}{\partial x_{1}}(0,0)\right|-\rho=\left(2^{5}-1\right) \rho
$$

$$
\frac{16\left|F\left(0, x^{\prime}\right)\right|}{\left|\frac{\partial F}{\partial x_{1}}\left(0, x^{\prime}\right)\right|^{2}} \leq \frac{16 \times 48.5}{31^{2}}<1 .
$$

Applying the lemma A.6.1, we get for all $\left|x^{\prime}\right| \leq \rho$ the existence of $\alpha\left(x^{\prime}\right)$ such that, when $\left|x_{1}\right| \leq 31 \rho / 4$

$$
\begin{gathered}
F\left(x_{1}, x^{\prime}\right)=\left(x_{1}+\alpha\left(x^{\prime}\right)\right) e(x), \quad\left|\alpha\left(x^{\prime}\right)\right| \leq \frac{33 \rho}{8}<5 \rho, \\
70 \rho \geq 8 \times 33 \rho / 4 \geq|e(x)| \geq 31 \rho / 4>7 \rho .
\end{gathered}
$$

The implicit function theorem guarantees the $C^{1}$ regularity of the function $\alpha$ and the Taylor-Lagrange formula with integral remainder provides the regularity of $e$.

REMark A.6.3. - If the function $F$ in the lemma A.6.2 is $C^{\infty}$, since the function $\alpha$ is obtained by the implicit function theorem, and $e$ by Taylor's formula with integral remainder, both function $\alpha, e$ are $C^{\infty}$. Moreover, the identity $F\left(-\alpha\left(x^{\prime}\right), x^{\prime}\right)=0$ implies that

$$
\left|\alpha^{(k)}\left(x^{\prime}\right)\right| \leq C_{k} \rho^{1-k}, \quad\left|e^{(k)}\left(x^{\prime}\right)\right| \leq C_{k} \rho^{-k}
$$

where $C_{k}$ are semi-norms of the function $F$ in $\max \left(\left|x_{1}\right|,\left|x^{\prime}\right|\right) \leq \rho$. In particular, if we apply this result to the function (2.1.21)

$$
F(T)=\Lambda^{1 / 2} q\left(t, Y+\nu(t, Y)^{1 / 2} T\right) \mu(t, Y)^{-1 / 2} \nu(t, Y)^{-1}
$$

BULLETIN DE LA SOCIÉTÉ MATHÉMATIQUE DE FRANCE 
we get that $\left|F^{(k)}\right|$ is bounded above by $\gamma_{k}(q)$ and $1 / 2 \leq \rho \leq \gamma_{1}(q)$ as defined in (2.1.1). We get then from the lemma A.6.2

$$
\Lambda^{1 / 2} q\left(t, Y+\nu(t, Y)^{1 / 2} T\right) \mu(t, Y)^{-1 / 2} \nu(t, Y)^{-1}=e_{0}(T)\left(T_{1}+\alpha_{0}\left(T^{\prime}\right)\right)
$$

so that $e_{0}, \alpha_{0}$ are smooth with fixed bounds and thus

$$
\begin{aligned}
& \Lambda^{1 / 2} q(t, X) \mu(t, Y)^{-1 / 2} \\
& \quad=e_{0}\left((X-Y) \nu(t, Y)^{-1 / 2}\right) \nu(t, Y)^{1 / 2} \times \\
& \quad \times\left(X_{1}-Y_{1}+\alpha_{0}\left(\left(X^{\prime}-Y^{\prime}\right) \nu(t, Y)^{-1 / 2}\right) \nu(t, Y)^{1 / 2}\right)
\end{aligned}
$$

which corresponds exactly to $(2.1 .22-23-24)$.

A.7. On tensor products of homogeneous functions. - Let $n \geq 1$ be an integer and $N=n+1$. Let $\left(y_{0} ; \eta_{0}\right) \in \mathbb{R}^{N} \times \mathbb{S}^{N-1}$ such that

$$
\left(y_{0} ; \eta_{0}\right)=\left(t_{0}, x_{0} ; \tau_{0}, \xi_{0}\right) \in \mathbb{R} \times \mathbb{R}^{n} \times \mathbb{R} \times \mathbb{R}^{n}, \quad \text { with } \tau_{0}=0, \xi_{0} \in \mathbb{S}^{n-1} .
$$

Let $r \in] 0,1 / 4]$ be given. There exists a function $\chi_{0} \in C^{\infty}\left(\mathbb{R}^{N} ;[0,1]\right)$ such that for $\lambda \geq 1$ and $\eta \in \mathbb{R}^{N}$ with $|\eta| \geq 1$, we have $\chi_{0}(\lambda \eta)=\chi_{0}(\eta)$ ("homogeneity of degree zero outside the unit ball") and

$$
\chi_{0}(\tau, \xi)= \begin{cases}1 & \text { if } \tau^{2}+|\xi|^{2} \geq 1 \text { and }|\tau| \leq r|\xi|, \\ 0 & \text { if } \tau^{2}+|\xi|^{2} \leq 1 / 4 \text { or }|\tau| \geq 2 r|\xi| .\end{cases}
$$

There exists a function $\psi_{0} \in C^{\infty}\left(\mathbb{R}^{n} ;[0,1]\right)$ such that for $\lambda \geq 1$ and $\xi \in \mathbb{R}^{n}$ with $|\xi| \geq 1$, we have $\psi_{0}(\lambda \xi)=\psi_{0}(\xi)$ and,

$$
\psi_{0}(\xi)= \begin{cases}1 & \text { if }|\xi| \geq 1 \text { and }\left|\frac{\xi}{|\xi|}-\xi_{0}\right| \leq r \\ 0 & \text { if }|\xi| \leq 1 / 2 \text { or }\left|\frac{\xi}{|\xi|}-\xi_{0}\right| \geq 2 r\end{cases}
$$

We define the function $\Phi_{0}$ by

$$
\Phi_{0}(\tau, \xi)=\chi_{0}(\tau, \xi) \psi_{0}(\xi) .
$$

LEMMA A.7.1. - The function $\Phi_{0}$ is such that for $\lambda \geq 1$ and $\eta \in \mathbb{R}^{N}$ with $|\eta| \geq 2$, we have $\Phi_{0}(\lambda \eta)=\Phi_{0}(\eta)$. Moreover, with $\eta_{0}=\left(0, \xi_{0}\right)$, we have

$$
\begin{gathered}
\Phi_{0}(\eta)=1 \text { for }|\eta| \geq 2 \text { and }\left|\frac{\eta}{|\eta|}-\eta_{0}\right| \leq r / 2, \\
\Phi_{0}(\eta)=0 \text { for }|\eta| \geq 2 \text { and }\left|\frac{\eta}{|\eta|}-\eta_{0}\right| \geq 4 r .
\end{gathered}
$$

Proof. - The function $\Phi_{0}$ is such that for $\lambda \geq 1$ and $\eta \in \mathbb{R}^{N}$ with $|\eta| \geq 2$, we have $\Phi_{0}(\lambda \eta)=\Phi_{0}(\eta)$ : in fact, if $\tau^{2}+|\xi|^{2} \geq 4$ and $|\tau| \leq 2 r|\xi|$, we get $|\xi|^{2} \geq 4\left(1+4 r^{2}\right)^{-1} \geq 1$, so that $\psi_{0}(\lambda \xi)=\psi_{0}(\xi)$ and since we have also in that case $\chi_{0}(\lambda \eta)=\chi_{0}(\eta)$, we get the sought property. Now if $\tau^{2}+|\xi|^{2} \geq 4$ and

TOME $134-2006-\mathrm{N}^{\mathrm{O}} 4$ 
$|\tau|>2 r|\xi|$, we see that $\chi_{0}(\lambda \tau, \lambda \xi)=\chi_{0}(\tau, \xi)=0$ so that, $\Phi_{0}(\lambda \eta)=0=\Phi_{0}(\eta)$. Moreover, if $\tau^{2}+|\xi|^{2} \geq 4$ and

$$
\frac{\tau^{2}}{\tau^{2}+|\xi|^{2}}+\left|\frac{\xi}{\left(\tau^{2}+|\xi|^{2}\right)^{1 / 2}}-\xi_{0}\right|^{2} \leq r^{2} / 4
$$

we get that $|\tau| \leq r|\xi|\left(4-r^{2}\right)^{-1 / 2} \leq r|\xi|$ and thus $\chi_{0}(\tau, \xi)=1$; also this implies $|\xi| \geq 2\left(1+r^{2}\right)^{-1 / 2} \geq 1$, so that $\psi_{0}(\xi)=\psi_{0}(\xi /|\xi|)$. We have then

$$
\left|\frac{\xi}{|\xi|}-\xi_{0}\right| \leq \frac{r}{2}+\left|\frac{\xi}{|\xi|}-\frac{\xi}{\left(\tau^{2}+|\xi|^{2}\right)^{1 / 2}}\right| \leq \frac{r}{2}+|\xi||\tau|^{2}|\xi|^{-3} \leq \frac{r}{2}+\frac{r^{2}}{4-r^{2}} \leq r,
$$

which implies $\psi_{0}(\xi)=\psi_{0}(\xi /|\xi|)=1$, so that $\Phi_{0}$ is equal to 1 on a conic neighborhood of $\left(0, \xi_{0}\right)$ in $\mathbb{R}^{N}$ minus a ball. Similarly, if $\tau^{2}+|\xi|^{2} \geq 4$ and

$$
\frac{\tau^{2}}{\tau^{2}+|\xi|^{2}}+\left|\frac{\xi}{\left(\tau^{2}+|\xi|^{2}\right)^{1 / 2}}-\xi_{0}\right|^{2} \geq 16 r^{2}
$$

either $|\tau| \geq 2 r|\xi|$ and $\chi_{0}(\tau, \xi)=0$, entailing $\Phi_{0}(\tau, \xi)=0$ or $|\tau| \leq 2 r|\xi|$ and then $\left|\frac{\xi}{\left(\tau^{2}+|\xi|^{2}\right)^{1 / 2}}-\xi_{0}\right|^{2} \geq 12 r^{2}$ and $|\xi| \geq 2\left(1+4 r^{2}\right)^{-1 / 2} \geq 1$ so that $\psi_{0}(\xi)=$ $\psi_{0}(\xi /|\xi|)$. In this case, we have

$$
\left|\frac{\xi}{|\xi|}-\xi_{0}\right| \geq 2 \sqrt{3} r-\left|\frac{\xi}{|\xi|}-\frac{\xi}{\left(\tau^{2}+|\xi|^{2}\right)^{1 / 2}}\right| \geq 2 \sqrt{3} r-\frac{\tau^{2}}{|\xi|^{2}} \geq 2 \sqrt{3} r-4 r^{2} \geq 2 r,
$$

implying $\psi_{0}(\xi)=0$ and thus $\Phi_{0}(\tau, \xi)=0$. Eventually, we have proven that $\Phi_{0}$ is also supported in a conic neighborhood of $\left(0, \xi_{0}\right)$ in $\mathbb{R}^{N}$.

\section{A.8. Composition of symbols}

LemmA A.8.1. - Let $G, g$ be the metrics on $\mathbb{R}^{2 N}$ defined in (4.3.8) and let $s_{1}, s_{2}$ be two real numbers. Let a be a symbol in $S\left(\langle\xi\rangle^{s_{1}}, g\right)$ and $b$ be a symbol in $S\left(\langle\xi, \tau\rangle^{s_{2}}, G\right)$ such that $\operatorname{supp} b \subset Z_{C}=\left\{(t, x, \tau, \xi) \in \mathbb{R}^{2 N},|\tau| \leq 1+C|\xi|\right\}$. Then the symbols $a \sharp b, b \sharp a, a \circ b, b \circ a$ belong to $S\left(\langle\xi, \tau\rangle^{s_{1}+s_{2}}, G\right)$ and are essentially supported in $Z_{C}$, i.e., are the sum of a symbol of $S\left(\langle\xi, \tau\rangle^{s_{1}+s_{2}}, G\right)$ supported in $Z_{C}$ and of a symbol in $S\left(\langle\xi, \tau\rangle^{-\infty}, G\right)=\cap_{N} S\left(\langle\xi, \tau\rangle^{-N}, G\right)$.

Proof. - We have

$$
\begin{aligned}
& (a \circ b)(t, x, \tau, \xi) \\
& \quad=\int e^{-2 i \pi(s \sigma+y \eta)} a(t, x, \tau+\sigma, \xi+\eta) b(t+s, x+y, \tau, \xi) d s d \sigma d y d \eta
\end{aligned}
$$


so that, using the standard expansion of the symbols and the fact that $b$ is supported in $Z_{C}$,

$$
\begin{aligned}
a \circ b=\sum_{|\alpha|<\nu} \frac{1}{\alpha !} \overbrace{D_{\tau, \xi}^{\alpha} a \partial_{t, x}^{\alpha} b}^{\in S(\langle\tau, \xi\rangle}+\int_{0}^{1-|\alpha|} \frac{(1-\theta)^{\nu-1}}{(\nu-1) !} & e^{-2 i \pi(s \sigma+y \eta)} D_{\tau, \xi}^{\nu} a(t, x, \tau+\theta \sigma, \xi+\theta \eta) \times \\
& \times \partial_{t, x}^{\nu} b(t+s, x+y, \tau, \xi) d s d \sigma d y d \eta d \theta .
\end{aligned}
$$

We define

$$
\begin{aligned}
I_{\theta}(\tau, \xi)=\int e^{-2 i \pi(s \sigma+y \eta)} D_{\tau, \xi}^{\nu} a(t, & x, \\
& \times \partial_{t, x}^{\nu} b(t+s, x+y, \tau, \xi) d s d \sigma d y d \eta
\end{aligned}
$$

and integrating by parts, we obtain for all nonnegative even integers $m$ that

$$
\begin{array}{r}
I_{\theta}(\tau, \xi)=\int e^{-2 i \pi(s \sigma+y \eta)}\langle\sigma\rangle^{-m}\left\langle D_{s}\right\rangle^{m}\langle s\rangle^{-m}\left\langle D_{\sigma}\right\rangle^{m}\langle y\rangle^{-m}\left\langle D_{\eta}\right\rangle^{m}\langle\eta\rangle^{-m}\left\langle D_{y}\right\rangle^{m} \\
D_{\tau, \xi}^{\nu} a(t, x, \tau+\theta \sigma, \xi+\theta \eta) \partial_{t, x}^{\nu} b(t+s, x+y, \tau, \xi) d s d \sigma d y d \eta,
\end{array}
$$

and consequently

$$
\left|I_{\theta}(\tau, \xi)\right| \lesssim \int\langle\sigma\rangle^{-m}\langle s\rangle^{-m}\langle y\rangle^{-m}\langle\eta\rangle^{-m}(1+|\xi+\theta \eta|)^{s_{1}-\nu} d s d \sigma d y d \eta
$$

$$
(1+|\xi|+|\tau|)^{s_{2}} \mathbf{1}(|\tau| \lesssim|\xi|) .
$$

In the integrand, when $|\eta| \leq|\xi| / 2$, we get, since $\theta \in[0,1],|\xi+\theta \eta| \geq|\xi|-|\eta| \geq$ $|\xi| / 2$. As a result, we get for this part of the integral the estimate

$(1+|\xi|)^{\left|s_{1}\right|-\nu}(1+|\xi|+|\tau|)^{s_{2}} \mathbf{1}(|\tau| \lesssim|\xi|) \lesssim(1+|\xi|+|\tau|)^{-\nu / 2}, \quad$ for $\nu$ large enough. When $|\eta|>|\xi| / 2$, we use the term $\langle\eta\rangle^{-m}$ and the estimate

$(1+|\xi|)^{-m / 2}(1+|\xi|+|\tau|)^{s_{2}} \mathbf{1}(|\tau| \lesssim|\xi|) \lesssim(1+|\xi|+|\tau|)^{-m / 4}, \quad$ for $m$ large enough.

To check that the derivatives of $I_{\theta}$ will satisfy the expected estimates, we differentiate the expression (A.8.2) and repeat the previous proof. We know now that, for $\nu$ large enough,

$$
a \circ b=\sum_{|\alpha|<\nu} \frac{1}{\alpha !} D_{\tau, \xi}^{\alpha} a \partial_{t, x}^{\alpha} b+r_{\nu}, \quad r_{\nu} \in S\left(\langle\tau, \xi\rangle^{-\nu / 2}, G\right) .
$$

Using the standard Borel argument, we find $c \in S\left(\langle\tau, \xi\rangle^{s_{1}+s_{2}}, G\right)$, essentially supported in $Z_{C}$ such that, for all $\nu$

$$
c-\sum_{|\alpha|<\nu} \frac{1}{\alpha !} D_{\tau, \xi}^{\alpha} a \partial_{t, x}^{\alpha} b \in S\left(\langle\tau, \xi\rangle^{s_{1}+s_{2}-\nu}, G\right),
$$

entailing that, for all $\nu \geq N_{0}$,

$$
a \circ b-c=-c+\sum_{|\alpha|<\nu} \frac{1}{\alpha !} D_{\tau, \xi}^{\alpha} a \partial_{t, x}^{\alpha} b+r_{\nu} \in S\left(\langle\tau, \xi\rangle^{\max \left(-\nu / 2, s_{1}+s_{2}-\nu\right)}, G\right),
$$

TOME $134-2006-\mathrm{N}^{\mathrm{O}} 4$ 
implying that $a \circ b-c \in S\left(\langle\tau, \xi\rangle^{-\infty}, G\right)$, which gives the result of the lemma for $a \circ b$. To get the result for $b \circ a$ is somewhat easier by looking at (A.8.2), to obtain the estimate

$$
\begin{aligned}
\left|I_{\theta}(\tau, \xi)\right| \lesssim \int\langle\sigma\rangle^{-m}\langle s\rangle^{-m}\langle y\rangle^{-m}\langle\eta\rangle^{-m}(1+|\xi+\theta \eta|+|\tau+\theta \sigma|)^{s_{2}-\nu} \\
\mathbf{1}(|\tau+\theta \sigma \eta| \lesssim|\xi+\theta \eta|) d s d \sigma d y d \eta(1+|\xi|)^{s_{1}} .
\end{aligned}
$$

When $|\tau| \lesssim|\xi|$ the discussion is the same as for $a \circ b$. When $|\tau| \gg|\xi|$, we split the integral in two parts: the region where $|\sigma| \leq|\tau| / 2$, in which we get negative powers of $(1+|\tau|)$ from the term with the exponent $s_{2}-\nu$, and the region where $|\sigma|>|\tau| / 2$ in which we use the term $\langle\sigma\rangle^{-m}$. The last part of the discussion is the same. To obtain the result for $a \sharp b$ (which will give also $b \sharp a$ since $\overline{a \sharp b}=\bar{b} \sharp \bar{a})$, we use the group $J^{t}=\exp 2 i \pi t D_{x} D_{\xi}$ and the formula $a \sharp b=J^{-1 / 2}\left(J^{1 / 2} a \circ J^{1 / 2} b\right)$. Using the assumptions of the lemma, we see that $J^{1 / 2} a$ satisfies the same hypothesis as $a$ and $J^{1 / 2} b$ is essentially supported in $Z_{C}$. The proofs above give thus that $J^{1 / 2} a \circ J^{1 / 2} b$ satisfies the conclusion of the lemma, which is "stable" by the action of $J^{-1 / 2}$. The proof of the lemma A.8.1 is complete.

\section{BIBLIOGRAPHY}

[1] BeAls (R.) \& FefFerman (C.) - On local solvability of linear partial differential equations, Ann. of Math., t. 97 (1973), pp. 482-498.

[2] Bony (J.M.) \& Chemin (J.Y.) - Espaces fonctionnels associés au calcul de Weyl-Hörmander, Bull Soc. Math. France, t. 122 (1994), pp. 77-118.

[3] Bony (J.M.) \& LeRner (N.) - Quantification asymptotique et microlocalisations d'ordre supérieur, Ann. Sci. Éc. Norm. Sup., t. 22 (1989), pp. $377-433$.

[4] Dencker (N.) - Estimates and solvability, Ark. Mat., t. 37 (1999), pp. 221-243.

[5] _ On the sufficiency of condition $(\psi)$, preprint, May 222001.

[6] _ The solvability of pseudo-differential operators, in Phase space analysis of partial differential equations, Pubbl. Cent. Ric. Mat. Ennio Giorgi, vol. 1, Sc. Norm. Sup., Pisa, 2004, pp. 175-200.

[7] t. 163 (2006), pp. 405-444.

[8] Fefferman (C.) \& PhOng (D.H.) - On positivity of pseudo-differential equations, Proc. Nat. Acad. Sci., t. 75 (1978), pp. 4673-4674.

[9] Hörmander (L.) - On the theory of general partial differential operators, Acta Math., t. 94 (1955), pp. 161-248.

[10] _ Differential equations without solutions, Math. Ann., t. 140 (1960), pp. 169-173.

BULletin DE LA SOCiÉtÉ MATHÉmATiQUE DE FRANCE 
[11] problems, Ann. of Math., t. 83 (1966), pp. 129-209.

[12] _ Propagation of singularities and semiglobal existence theorems for (pseudo-)differential operators of principal type, Ann. of Math., t. 108 (1978), pp. 569-609.

[13] _ Pseudo-differential operators of principal type, in Singularities in boundary value problems, D. Reidel Publ. Co., Dortrecht, Boston, London, 1981.

[14] Verlag, 1983-85.

[15] _ Notions of convexity, Birkhäuser, 1994.

[16] _ On the solvability of pseudodifferential equations, in Structure of solutions of differential equations (Morimoto (M.) \& Kawai (T.), eds.), World Sci. Publishing, River Edge, NJ, 1996, pp. 183-213.

[17] _ private communications, september 2002 - august 2004.

[18] Lerner (N.) - Sufficiency of condition $(\psi)$ for local solvability in two dimensions, Ann. of Math., t. 128 (1988), pp. 243-258.

[19] _ An iff solvability condition for the oblique derivative problem, Séminaire EDP, École polytechnique, 1990-91, exposé 18.

[20] Nonsolvability in $L^{2}$ for a first order operator satisfying condition ( $\psi)$, Ann. of Math., t. 139 (1994), pp. 363-393.

[21] _ Energy methods via coherent states and advanced pseudodifferential calculus, in Multidimensional complex analysis and partial differential equations (Cordaro (P.D.), Jacobowitz (H.) \& Gindikin (S.), eds.), Amer. Math. Soc., 1997, pp. 177-201.

[22] _ Perturbation and energy estimates, Ann. Sci. Éc. Norm. Sup., t. 31 (1998), pp. 843-886.

[23] When is a pseudo-differential equation solvable?, Ann. Inst. Fourier (Grenoble), t. 50 (2000), pp. 443-460.

[24] Solving pseudo-differential equations, in Proceedings of the ICM 2002 in Beijing, vol. II, Higher Education Press, 2002, pp. 711-720.

[25] LEWy (H.) - An example of a smooth linear partial differential equation without solution, Ann. of Math., t. 66 (1957), pp. 155-158.

[26] Mizohata (S.) - Solutions nulles et solutions non analytiques, J. Math. Kyoto Univ., t. 1 (1962), pp. 271-302.

[27] Moyer (R.D.) - Local solvability in two dimensions: necessary conditions for the principal type case, mimeographed manuscript, University of Kansas, 1978.

[28] Nirenberg (L.) \& Treves (F.) - Solvability of a first order linear partial differential equation, Comm. Pure Appl. Math., t. 16 (1963), pp. 331-351.

TOME $134-2006-\mathrm{N}^{\mathrm{O}} 4$ 
[29] _ On local solvability of linear partial differential equations. I. Necessary conditions, Comm. Pure Appl. Math., t. 23 (1970), pp. 1-38.

[30] - On local solvability of linear partial differential equations. II.Sufficient conditions, Comm. Pure Appl. Math., t. 23 (1970), pp. 459509.

[31] _ On local solvability of linear partial differential equations. Correction, Comm. Pure Appl. Math., t. 24 (1971), pp. 279-288.

[32] Trépreau (J.-M.) - Sur la résolubilité analytique microlocale des opérateurs pseudo-différentiels de type principal, Ph.D. Thesis, Université de Reims, 1984. 